space holder for signature page 


\title{
A Search for Chargino-Neutralino Production at the Fermilab Tevatron Collider
}

\author{
by \\ Benn Tannenbaum \\ B.A., Physics, Grinnell College, 1990 \\ M.S., Physics, Michigan State University, 1993
}

Doctor of Philosophy

Physics

December, 1997 
4.3 Distributions for CEM electrons which pass the loose ID cuts. . . . . . . . . . . . . 55

4.4 Distributions for CEM electrons which pass the loose ID cuts. . . . . . . . . . . . . 56

4.5 Distributions for PEM electrons which pass the loose ID cuts. . . . . . . . . . . . . 57

4.6 Two body mass for opposite sign electron pairs. . . . . . . . . . . . . . . . . . . . . 58

4.7 Distributions for CMU/P muons which pass the tight ID cuts. . . . . . . . . . . . . 60

4.8 Distributions for CMU/P muons which pass the tight ID cuts. . . . . . . . . . . . . 61

4.9 Distributions for CMU/P muons which pass the loose ID cuts. . . . . . . . . . . . . . 62

4.10 Distributions for CMU/P muons which pass the loose ID cuts. . . . . . . . . . . . . 63

4.11 Distributions for CMX muons which pass the loose ID cuts. . . . . . . . . . . . . . . 64

4.12 Distributions for CMX muons which pass the loose ID cuts. . . . . . . . . . . . . . 65

4.13 Distributions for CMIO muons which pass the loose ID cuts. . . . . . . . . . . . . . 66

4.14 Two body mass for opposite sign muon pairs. . . . . . . . . . . . . . . . . . . . . 67

4.15 Two body mass for opposite sign muon pairs in the $J / \psi$ region. . . . . . . . . . . . . 68

4.16 Cosmic ray filter status bit for candidate CMU/P and CMX muons. . . . . . . . . . 72

4.17 Event display for a typical cosmic ray. . . . . . . . . . . . . . . . . . . . . . 73

4.18 Maximum and middle opening angles for the three possible lepton pair combinations for a sample Monte Carlo point. . . . . . . . . . . . . . . . . . . . . . . . . 74

4.19 Maximum and middle opening angles for the three possible lepton pair combinations for $Z^{0} \rightarrow \mu \mu$ events with a third $\mu$ (data) . . . . . . . . . . . . . 75

4.20 Maximum and middle opening angles for the three possible lepton pair combinations for the trilepton data set. . . . . . . . . . . . . . . . . . . . 75

4.21 The difference in time for muon pairs. . . . . . . . . . . . . . . . . 76

4.22 The difference in timing for $Z^{0} \rightarrow \mu \mu$ events (from data) with a third muon in the event. . . . . . . . . . . . . . . . . . 77

4.23 Isolation for leptons from $b$ quark decays. . . . . . . . . . . . . . . . . . . . . . . 79

4.24 Opening angle for lepton pairs from $b$ quark decays. . . . . . . . . . . . . . . . . 80

4.25 Two-body masses for diboson background events. . . . . . . . . . . . . . . . . . . . 82

4.26 Opening angle for background due to Drell-Yan processes. . . . . . . . . . . . . . . 84

4.27 Dilepton mass for background due to Drell-Yan processes. . . . . . . . . . . . . . . . 85

4.28 Missing transverse energy for each of the types of background. . . . . . . . . . . 86

5.1 The detection efficiency as a function of $\mathrm{M}\left(\tilde{\chi}_{1}^{ \pm}\right) \ldots \ldots \ldots \ldots$. . . . . . . . . . 94

5.2 The detection efficiency as a function of $\mathrm{M}\left(\tilde{\chi}_{1}^{ \pm}\right)$for a variety of points in SiMSSM

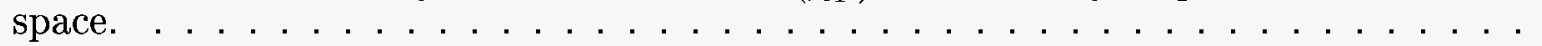

5.3 The detection efficiency as a function of $\mathrm{M}\left(\tilde{\chi}_{1}^{ \pm}\right)$comparing individual SiMSSM points to an overall fit. . . . . . . . . . . . . . . . . . . . . . 96 
5.4 The detection efficiency as a function of $\mathrm{M}\left(\tilde{\chi}_{1}^{ \pm}\right)$for a variety of points in SiMSSM

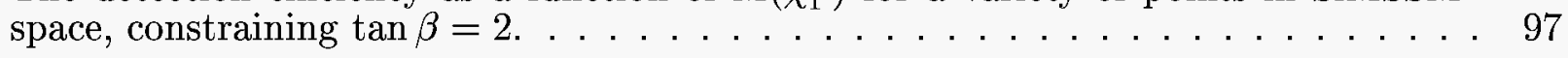

5.5 Production cross section times branching ratio to leptons for $\tilde{\chi}_{1}^{ \pm} \tilde{\chi}_{2}^{0}$ production as a function of $\tilde{\chi}_{1}^{ \pm}$mass for $\tan \beta=2$, and $\mu=-400 \mathrm{GeV} / \mathrm{c}^{2} \ldots \ldots \ldots 101$

5.6 Production cross section times branching ratio to leptons for $\tilde{\chi}_{1}^{ \pm} \tilde{\chi}_{2}^{0}$ production as a function of $\tilde{\chi}_{1}^{ \pm}$mass for light squarks. . . . . . . . . . . . . . . . . 102

5.7 Lower limit on $\mathrm{M}\left(\tilde{\chi}_{1}^{ \pm}\right)$as a function of $\mathrm{M}(\tilde{q}) / \mathrm{M}(\tilde{g}) \ldots \ldots \ldots \ldots \ldots$

5.8 Production cross section times branching ratio to leptons for $\tilde{\chi}_{1}^{ \pm} \tilde{\chi}_{2}^{0}$ production as a function of $\tilde{\chi}_{1}^{ \pm}$mass for $\tan \beta=2$, and $\mathrm{M}(\tilde{q})=\mathrm{M}(\tilde{g}) \ldots \ldots \ldots 4$

5.9 Lower limit of $\mathrm{M}\left(\tilde{\chi}_{1}^{ \pm}\right)$as a function of $\mu \ldots \ldots \ldots \ldots \ldots \ldots$

5.10 Production cross section times branching ratio to leptons for $\tilde{\chi}_{1}^{ \pm} \tilde{\chi}_{2}^{0}$ production as a function of $\tilde{\chi}_{1}^{ \pm}$mass for $\tan \beta=1.1, \mathrm{M}(\tilde{q})=\mathrm{M}(\tilde{g})$ and $\mu=-400 \mathrm{GeV} / \mathrm{c}^{2} \ldots \ldots 106$

5.11 Production cross section times branching ratio to leptons for $\tilde{\chi}_{1}^{ \pm} \tilde{\chi}_{2}^{0}$ production as a function of $\tilde{\chi}_{1}^{ \pm}$mass for $\tan \beta=1.5, \mathrm{M}(\tilde{q})=\mathrm{M}(\tilde{g})$ and $\mu=-400 \mathrm{GeV} / \mathrm{c}^{2} \ldots \ldots 107$

5.12 Production cross section times branching ratio to leptons for $\tilde{\chi}_{1}^{ \pm} \tilde{\chi}_{2}^{0}$ production as a function of $\tilde{\chi}_{1}^{ \pm}$mass for $\tan \beta=2, \mathrm{M}(\tilde{q})=\mathrm{M}(\tilde{g})$ and $\mu=-400 \mathrm{GeV} / \mathrm{c}^{2} \ldots \ldots 108$

5.13 Production cross section times branching ratio to leptons for $\tilde{\chi}_{1}^{ \pm} \tilde{\chi}_{2}^{0}$ production as a function of $\tilde{\chi}_{1}^{ \pm}$mass for $\tan \beta=4, \mathrm{M}(\tilde{q})=\mathrm{M}(\tilde{g})$ and $\mu=-400 \mathrm{GeV} / \mathrm{c}^{2} \ldots \ldots 109$

5.14 Production cross section times branching ratio to leptons for $\tilde{\chi}_{1}^{ \pm} \tilde{\chi}_{2}^{0}$ production as a function of $\tilde{\chi}_{1}^{ \pm}$mass for $\tan \beta=8, \mathrm{M}(\tilde{q})=\mathrm{M}(\tilde{g})$ and $\mu=-400 \mathrm{GeV} / \mathrm{c}^{2} \ldots \ldots 110$

5.15 Lower limit on $\mathrm{M}\left(\tilde{\chi}_{1}^{ \pm}\right)$as a function of $\tan \beta \ldots \ldots \ldots \ldots \ldots \ldots \ldots$

5.16 Production cross section times branching ratio to leptons for $\tilde{\chi}_{1}^{ \pm} \tilde{\chi}_{2}^{0}$ production as a function of $\tilde{\chi}_{1}^{ \pm}$mass for $\tan \beta=2$ and $\mu=-400 \mathrm{GeV} / \mathrm{c}^{2}$ and $\mathrm{M}(\tilde{q})=500 \mathrm{GeV} / \mathrm{c}^{2} \ldots 112$

5.17 The acceptance for all four trilepton modes for $\mathrm{SU}(5) \times \mathrm{U}(1)$ model production of trileptons. . . . . . . . . . . . . . . . . . . . . 118

$5.18 \sigma \cdot B R$ versus $\tilde{\chi}_{1}^{ \pm}$mass for $\mathrm{SU}(5) \times \mathrm{U}(1)$ model production of trileptons. . . . . . 119

5.19 Detection efficiency using the SUGRA model. . . . . . . . . . . . . . . . . . 122

5.20 Production cross section times branching ratio to leptons for $\tilde{\chi}_{1}^{ \pm} \tilde{\chi}_{2}^{0}$ production as a

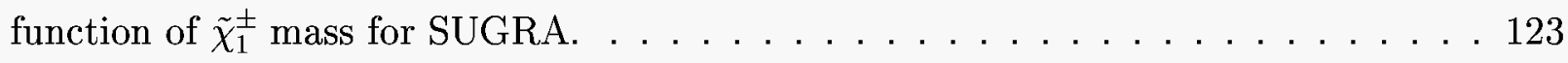


6.1 The naturalness of a variety of different sparticles. . . . . . . . . . . . . 130

6.2 Production cross section for a variety of processes at the upgraded Tevatron. . . . . 132 


\section{List of Tables}

1.1 The fermionic particles of the Standard Model. . . . . . . . . . . . . . . . . . . . . 2

1.2 The bosonic particles of the Standard Model. . . . . . . . . . . . . . . . . . . . . 2

2.1 Standard Model and SUSY particle spectrum. . . . . . . . . . . . . . . . . . . 27

3.1 Summary of the properties of the various CDF calorimeter systems. . . . . . . . . . 38

3.2 Summary of efficiencies for lepton IDs. . . . . . . . . . . . . . . . . . . . . . . . 45

4.1 Skimming selection criteria for CEM and PEM electrons. . . . . . . . . . . . . . . 50

4.2 Skimming selection criteria for CMU/P, CMX and CMIO muons. . . . . . . . . . . . 50

4.3 Number of CEM and PEM electrons left after each lepton identification cut. . . . . . 52

4.4 Number of CMU/P, CMX and CMIO muons left after each lepton identification cut. 59

4.5 Analysis lepton selection cuts for CEM and PEM electrons. . . . . . . . . . . . . . 65

4.6 Analysis lepton selection cuts for CMU/P, CMX and CMIO muons. . . . . . . . . . 69

4.7 Number of CEM and PEM electrons left after each analysis lepton identification cut. 69

4.8 Number of CMU/P, CMX and CMIO muons left after each analysis lepton identification cut. . . . . . . . . . . . . . . . . . . . . . 74

4.9 Expected background after each cut. . . . . . . . . . . . . . . . . . . . 87

4.10 Number of data, expected background and signal Monte Carlo after each cut. . . . . 88

5.1 The Level 2 triggers used in this analysis. . . . . . . . . . . . . . . . . . . . . . . 91

5.2 Input and output parameters for the SiMSSM points scanned. . . . . . . . . . . . 113

5.3 Input and output parameters for the SiMSSM points scanned (cont.). . . . . . . . . 114

5.4 Input and output parameters for the SiMSSM points scanned (cont.). . . . . . . . . 115

5.5 Input and output parameters for the SiMSSM points scanned (cont.). . . . . . . . . 116

5.6 Input and output parameters for the SiMSSM points scanned (cont.). . . . . . . . . 117

5.7 The input and output parameters used to examine the $\mathrm{SU}(5) \times \mathrm{U}(1)$ model. . . . . 120

5.8 Masses of the $\tilde{\chi}_{1}^{ \pm}, \tilde{\chi}_{2}^{0}, \tilde{\chi}_{1}^{0}$ for the points examined in the $\mathrm{SU}(5) \times \mathrm{U}(1)$ model. . . . 120

5.9 Input and output parameters for the SUGRA points scanned. . . . . . . . . . . . . . 124

5.10 Input and output parameters for the SUGRA points scanned. . . . . . . . . . . . . 125

5.11 Output parameters for the SUGRA points scanned. . . . . . . . . . . . . . . 126

5.12 Output parameters for the SUGRA points scanned (cont.). . . . . . . . . . . . . 127

5.13 Output parameters for the SUGRA points scanned (cont.). . . . . . . . . . . . . 128 
6.1 Limits on $\tilde{\chi}_{1}^{ \pm}$and $\tilde{\chi}_{2}^{0}$ masses in the SiMSSM framework. . . . . . . . . . . 131

6.2 Limits on $\tilde{\chi}_{1}^{ \pm}$and $\tilde{\chi}_{2}^{0}$ masses in the $\mathrm{SU}(5) \times \mathrm{U}(1)$ framework. . . . . . . . . 131

6.3 Limit on $\tilde{\chi}_{1}^{ \pm}$and $\tilde{\chi}_{2}^{0}$ masses in the Minimal SUGRA model. . . . . . . . . . . . 131

6.4 Lower limits for several searches during Run II. . . . . . . . . . . . . . . . . 133 


\section{Chapter 1}

\section{Introduction}

phys.ics (fiz'iks) $n$. the science dealing with the properties, changes, interaction, etc. of matter and energy in which energy is considered to be continuous (classical physics), including electricity, heat, optics, mechanics, etc., and now also dealing with the atomic scale of nature in which energy is considered to be discrete (quantum physics), including such branches as atomic, nuclear, and solid-state physics [1]

Particle, or high energy, physics, is the study of quarks and leptons and their interactions. Just as cells compose living organisms, atoms compose molecules and protons, neutrons and electrons compose atoms, the various quarks compose neutrons and protons. The theory describing the quarks and leptons is known as the Standard Model. Just as Mendeleev's periodic table organizes the elements into columns and rows according to composition, the Standard Model groups the quarks and leptons into families by charge and mass. Tables 1.1 and 1.2 list the known particles. In addition to the quarks and leptons, there are particles which mediate the four known forces: the photon $(\gamma)$ mediates the electromagnetic force, the $W^{ \pm}$and $Z^{0}$ mediate the the weak force, the gluon $(g)$ mediates the strong force and the graviton mediates gravity ${ }^{1}$.

The Standard Model is the combination of two other theories, the electroweak theory of Weinberg, Salam and Glashow (WSG), and Quantum Chromodynamics (QCD). QED, the part of the electroweak theory that describes the interactions of electrons, muons and taus with photons, is one of the most successful theories in all of science. The remainder of the WSG theory describes the charged and neutral weak interactions. QCD describes the interaction of quarks via the strong force.

\footnotetext{
${ }^{1}$ The graviton is a postulated particle which has not yet been seen. Since gravity is significantly weaker than the other three forces, its mediator is much more difficult to detect.
} 
Table 1.1: The fermionic particles of the Standard Model. The quarks in the top row have charge $+\frac{2}{3}$ times the electron charge, while the quarks in the second row have charge $-\frac{1}{3}$ times the electron charge. All of the leptons in the third row have charge -1 and all of the neutrinos are neutral. The quarks can interact via any of the four forces, the leptons via all but the strong force, and the neutrinos can interact via the weak and gravitational forces. All particles have been observed experimentally except the $\nu_{\tau}$.

\begin{tabular}{|c|cc|cc|cc|}
\hline quarks & up & $u$ & charm & $c$ & top & $t$ \\
& down & $d$ & strange & $s$ & bottom & $b$ \\
leptons & electron & $e$ & muon & $\mu$ & tau & $\tau$ \\
& electron neutrino & $\nu_{e}$ & muon neutrino & $\nu_{\mu}$ & tau neutrino & $\nu_{\tau}$ \\
\hline
\end{tabular}

Table 1.2: The bosonic particles of the Standard Model. All bosons have been observed except the graviton (G).

\begin{tabular}{|cl|}
\hline Boson & Force Mediated \\
\hline photon $(\gamma)$ & Electromagnetic \\
$W^{ \pm}$ & Charged Weak \\
$Z^{0}$ & Neutral Weak \\
gluon $(g)$ & Strong \\
graviton $(G)$ & Gravity \\
\hline
\end{tabular}

Overall, the Standard Model has been very successful. However, it leaves many questions unanswered. Many theories have been proposed to answer these questions. This dissertation is a search for evidence of physics beyond the Standard Model. Chapter 2 describes the theory examined, Chapter 3 the apparatus used, Chapter 4 the analysis techniques and Chapter 5 the limits reached. Chapter 6 draws some conclusions. 


\section{Chapter 2}

\section{Theory and Motivation}

The Standard Model has proven to be a very effective low energy ${ }^{1}$ theory- a theory that is accurate in the currently accessible energy spectrum. Its two major components, Quantum Chromodynamics (QCD) [2] and the electroweak theory of Weinberg, Salam and Glashow [3], predict the interaction of quarks, leptons and the gauge bosons well. The theory is so successful that the existence of the top quark was accepted long before its discovery. The production cross sections $(\sigma)$ and decay branching ratios for the various interactions are accurately predicted. In fact, there is very little experimental evidence for any physics beyond the Standard Model.

There are, however, many unanswered questions about the Standard Model and the universe in which we live. Many of these questions deal with mass. For example,

- Depending on the parameterization used, either the masses of all of the quarks and leptons or the couplings of the leptons and quarks to the vacuum expectation value of the Higgs boson are free. Why? Why does the Standard Model not predict the masses of the particles? Why are there three generations?

- The mass of the top quark is $176 \mathrm{GeV} / \mathrm{c}^{2}$ [4]. The mass of the other quark in its isospin doublet, the bottom quark, is $\simeq 4.5 \mathrm{GeV} / \mathrm{c}^{2}$. The ratio of the top to bottom mass is almost 40 ! The ratio of charm to strange mass is $\simeq 7.5$ and the ratio of up to down mass is $\simeq 0.5$ [5]. Why is the top quark so massive, and why is it so much more massive than its isospin partner?

- The mediator of the electromagnetic force, the photon, and the mediator of the strong force, the gluon, are both massless. Why are the mediators of the weak force, the $W^{ \pm}$and the $Z^{0}$,

\footnotetext{
1 "Low energy", of course, depends on the beholder. For this dissertation low energy implies energies of the TeV $\left(10^{12} \mathrm{eV}\right)$ scale or less.
} 


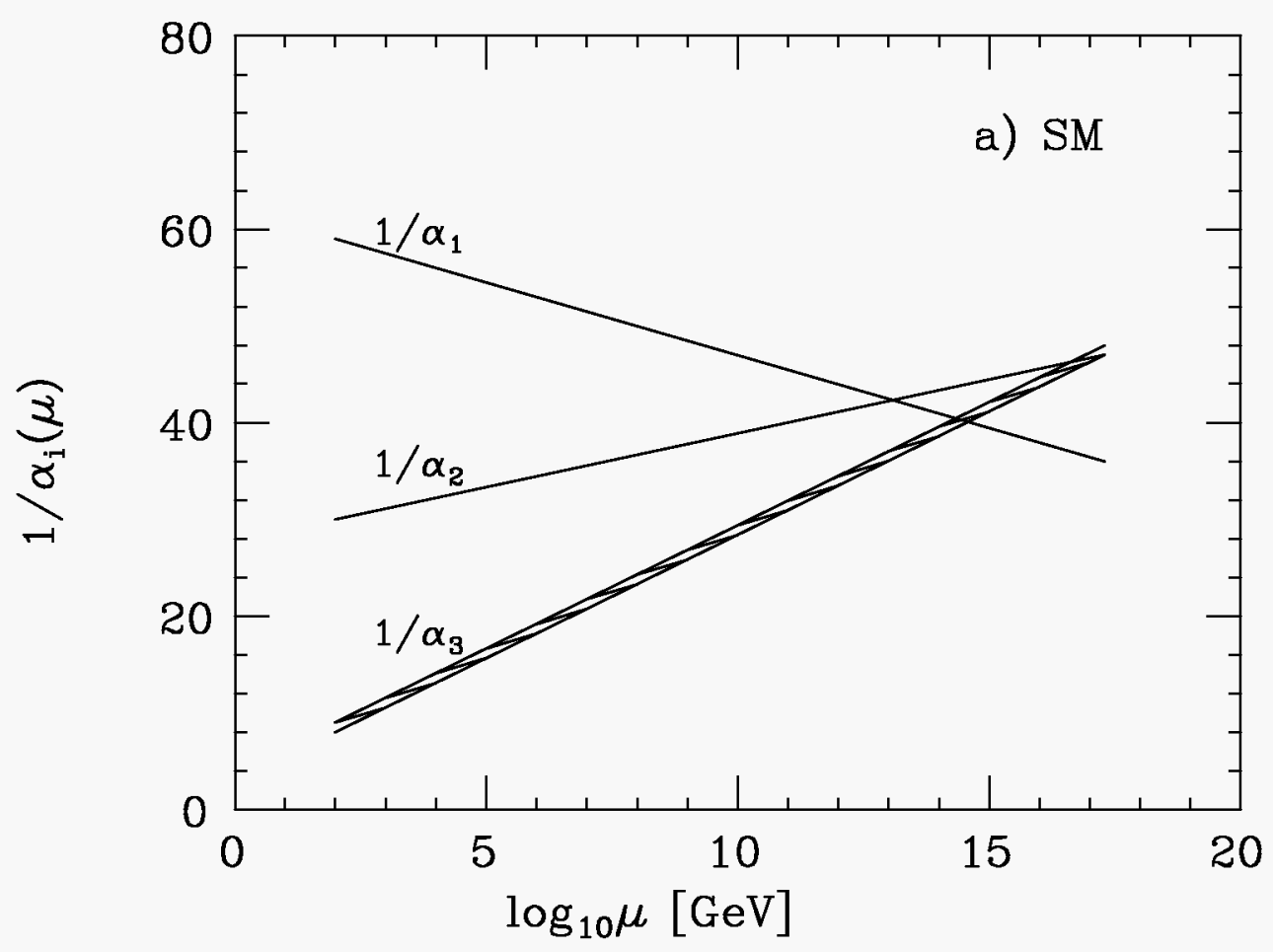

Figure 2.1: The extrapolation of the Standard Model gauge couplings to very high energies. $\alpha_{1}$ is the $\mathrm{SU}(1)_{\text {hypercharge }}$ coupling constant, $\alpha_{2}$ is the $\mathrm{SU}(2)_{\text {weak }}$ coupling constant and $\alpha_{3}$ is the $\mathrm{SU}(3)_{\text {strong }}$ coupling constant. Adapted from [6].

massive? Why are they so massive? What sets the scale for electroweak symmetry breaking?

- The Standard Model does not need to include the Higgs boson. The Higgs mechanism is inserted ad hoc to provide a mechanism for electroweak symmetry breaking and further gives masses to the quarks and leptons. It is a mechanism that works, but it may not be the right one.

- Early physics research led to the realization that electricity and magnetism are different aspects of the same force. The prediction and subsequent discovery of the $W^{ \pm}$and $Z^{0}$ led to the realization that, above certain energies, the electromagnetic and weak forces are different aspects of the same force. However, the SM-based extrapolation of precise measurements at LEP clearly show (Figure 2.1) that the strong, electromagnetic and weak forces do not unify at any energy. They do approach a common value, but do not meet at a point. We currently believe that there must exist a theory of everything in which all the forces unify: a Grand Unified Theory (GUT). This is not possible within the framework of the SM.

- Grand Unified Theories require a scalar particle to provide the mechanism for breaking the 
symmetry at the Grand Unified scale. However, its mass (on the order of the GUT scale: $10^{16} \mathrm{GeV} / \mathrm{c}^{2}$ ) must be specified to great accuracy to provide for the physics at the weak scale. This is known as the fine tuning or hierarchy problem.

- Current measurements of the rate of rotation of some distant galaxies do not match with the visible mass. This appears to imply that there must be some additional, unseen, mass that allows these galaxies to rotate as quickly as they do.

All of these facts lead us to believe that there must be a theory beyond the Standard Model. Many have been proposed, such as quark compositness (or technicolor), left-right symmetric models and SUperSYmmetry (SUSY). Each of these models solves some or all of the above problems and each has their own set of strengths and weaknesses. A weakness common to all of them is a total lack of experimental evidence for any of them. At present SUSY is a very popular theory and is generating interest in both the theoretical and experimental communities.

In the remainder of this Chapter, I will briefly describe the basic principles of SUSY and why SUSY is of interest, describe the particle spectrum, discuss signals and backgrounds germane to this analysis, and discuss naturalness.

\subsection{Overview of Supersymmetry}

The theory behind SUSY is complex and a complete introduction is beyond the scope of this dissertation. A quick search of the SPIRES data base [7] lists over 7,000 papers on the topic of SUSY. A good starting point for further reading can be found in Refs. $[8,9,10]$ and the references within. However, an introduction is appropriate, especially as there are few references that are comprehensible to the experimentalist at first reading: the transition from the SUSY Lagrangian to the constrained model we use is difficult.

\subsubsection{The Minimal Supersymmetric Model}

In the SM, each particle is described by several quantum numbers: spin, charge, mass, isospin, etc. All quarks and leptons are fermions, meaning they have half-integral spin. The mediators of the various forces are all bosons and thus have integral spin. SUSY supposes that for each particle with an integral spin, there exists a particle with the same charge, isospin, etc., but with half-integral spin. Likewise, for each half-integral spin particle there exists an integral spin partner. The names of these new particles are related to the names of the original particles: the scalar partners to the fermions are the sfermions (such as sleptons and squarks) and the partners to the gauge bosons gain an -ino (such as Higgsino). The Minimal Supersymmetric Model (MSSM) gives each particle a superpartner and requires two Higgs doublets and their superpartners. This more than doubles the 
particle spectrum and greatly increases the number of free parameters to over 100. A few reasonable assumptions (described in the following sections) reduce the number of free parameters to $\operatorname{six}^{2}$.

The second Higgs doublet is necessary to cancel anomalies [8]. The second doublet also allows one to use one doublet to give masses to the up-type quarks and the neutrinos, and the other doublet to give masses to the down-type quarks and the charged leptons.

\subsubsection{Reducing the parameter space}

The MSSM in its purest form has over 100 free parameters. One of my complaints about the SM was that it had too many free parameters. By adding SUSY I seem to have made the problem worse! To reduce the number of free parameters we can make certain model assumptions that are either based on observed or desired physics. The resulting model is unfortunately also sometimes known as the MSSM. I shall refer to it as the SiMSSM (Supergravity-inspired MSSM).

\section{Grand Unified Theories (GUTs)}

If we require unification of the coupling constants at the GUT scale ("the GUT hypothesis" provided by supergravity [11]), we are led to the following relationships:

$$
\left.\begin{array}{l}
M_{\widetilde{W}}=\frac{\alpha_{2}}{\alpha_{3}} M_{\tilde{g}} \\
M_{\widetilde{B}}=\frac{5 \alpha_{1}}{3 \alpha_{2}} M_{\widetilde{W}}
\end{array}\right\}
$$

where $\widetilde{W}$ and $\widetilde{B}$ are the weak eigenstates of the SUSY partners to the SM electroweak gauge bosons and the $\alpha_{i}$ are the hypercharge, weak isospin, and strong coupling constants at the appropriate scale, respectively. At the Tevatron scale $(\sqrt{s}=1.8 \mathrm{TeV}) \alpha_{1}=0.00781, \alpha_{2}=0.03397$ and $\alpha_{3}=$ 0.120 [5]. The $5 / 3$ arises from the use of $\mathrm{SU}(5)$ as the unifying group. $\mathrm{SU}(5)$ is the smallest group that can unify $\mathrm{U}(1) \times \mathrm{SU}(@) \times \mathrm{SU}(3)$ [12]. Equation 2.1 causes $\mathrm{M}_{\widetilde{W}}$ and $\mathrm{M}_{\widetilde{B}}$ to be functions of only $\mathrm{M}_{\tilde{g}}$, since the $\alpha_{i}$ are well measured quantities.

\section{Renormalization Group Equations}

We relate the slepton $(\widetilde{\ell})$ and sneutrino $(\widetilde{\nu})$ masses to the squark $(\tilde{q})$ and gluino $(\tilde{g})$ masses through the Renormalization Group Equations as inspired by supergravity models [13]. These relations are made at the GUT scale and must be rescaled (or renormalized) to the $\mathrm{TeV}$ scale where we currently

\footnotetext{
${ }^{2}$ See $e . g$. Ref. [10] for more details.
} 
work:

$$
\left.\begin{array}{l}
M_{\tilde{\ell}_{L}}^{2}=M_{\tilde{q}}^{2}-0.73 M_{\tilde{g}}^{2}-0.27 M_{Z}^{2} \cos 2 \beta \\
M_{\tilde{\ell}_{R}}^{2}=M_{\tilde{q}}^{2}-0.78 M_{\tilde{g}}^{2}-0.23 M_{Z}^{2} \cos 2 \beta \\
M_{\tilde{\nu}_{L}}^{2}=M_{\tilde{q}}^{2}-0.73 M_{\tilde{g}}^{2}+0.5 M_{Z}^{2} \cos 2 \beta
\end{array}\right\}
$$

We assume the masses of all generations of each type of slepton $\left(\widetilde{\ell}_{L}, \widetilde{\ell}_{R}\right.$ and $\left.\widetilde{\nu}_{L}\right)$ to be degenerate. These relations are calculated by averaging similar equations which do not assume degenerate generations [14]. This is acceptable since this analysis is largely affected by the masses of only the first generation squarks. The masses of the $\tilde{\ell}_{s}$ depend mainly on the difference in mass of the $\tilde{q} s$ and $\tilde{g} \mathrm{~s}$.

\section{$R$-parity and the Lightest Supersymmetric Partner}

The MSSM allows the imposition of a global symmetry, so we assign each particle and superparticle a new multiplicative quantum number, $R \equiv(-)^{B+L+2 S}$ where $B$ is the baryon number, $L$ is the lepton number and $S$ is the spin of the particle. Thus, SM particles have $R=+1$ and SUSY particles have $R=-1$. If we require $R$-parity to be conserved, we then require the creation of SUSY particles in pairs. By requiring SUSY particles to be created in pairs, we must then have a lightest sparticle (LSP) that is stable. An electrically or color charged LSP causes many problems [15] so the LSP is generally assumed to be the lightest neutralino $\left(\tilde{\chi}_{1}^{0}\right)$. Experimentally, the LSP can be thought of as a heavy neutrino: it will manifest itself as missing energy. The LSP is also an excellent candidate for cold dark matter [16].

\section{Remaining free parameters}

To continue reducing the number of free parameters, we further assume that the first and second generation squarks are degenerate in mass. This is an acceptable assumption, as this analysis is mainly affected by the masses of the first generation squarks. After making all the above assumptions, we are left with only a handful of free parameters: $\tan \beta, \mu, \mathrm{M}_{\tilde{g}}, \mathrm{M}_{\tilde{q}}, \mathrm{~A}_{T}$, and $\mathrm{M}_{H_{A}} \cdot \tan \beta$ is the ratio of the vacuum expectation values for the two Higgs doublets:

$$
\begin{aligned}
\tan \beta & =\left(\left\langle H_{2}\right\rangle /\left\langle H_{1}\right\rangle\right) \\
v^{2} & =\left\langle H_{1}\right\rangle^{2}+\left\langle H_{2}\right\rangle^{2}
\end{aligned}
$$

where $v=246 \mathrm{GeV}$ is fixed by the $W^{ \pm}$mass. $\tan \beta$ serves mainly to control the Higgsino vs gaugino content of the charginos $\left(\tilde{\chi}_{i}^{ \pm}\right)$and neutralinos $\left(\tilde{\chi}_{i}^{0}\right)$ (see Section 2.2 for explanations of these particles). $\mu$ is the un-mixed Higgsino mass (sometimes referred to as the supersymmetryconserving Higgs mass parameter) and sets the scale for SUSY. It also has an effect on the Higgsino vs gaugino content of the charginos and neutralinos. $\mathrm{M}_{\tilde{g}}$ is the gluino mass and $\mathrm{M}_{\tilde{q}}$ is the common 
squark mass. $A_{T}$ is the Higgs-squark-squark trilinear interaction for the top squark, and $\mathrm{M}_{\mathrm{H}_{\mathrm{A}}}$ is the mass of the pseudoscalar Higgs boson.

This analysis, which is a search for $\tilde{\chi}_{1}^{ \pm} \tilde{\chi}_{2}^{0}$ production, is obviously concerned with their masses and couplings. As will be seen below, once we know the masses and couplings of $\tilde{\chi}_{1}^{ \pm}, \tilde{\chi}_{1}^{0}, \tilde{\chi}_{2}^{0}$ and sleptons (and to some degree, the squarks), we can begin to set limits on the production of SUSY particles.

In the regime where $M_{Z^{0}} \ll\left|M_{i} \pm \mu\right|$ the masses of $\tilde{\chi}_{1}^{ \pm}, \tilde{\chi}_{1}^{0}$ and $\tilde{\chi}_{2}^{0}$ are functions solely of $\mu$, $\tan \beta$ and the mass of the gluino [17]:

$$
\begin{gathered}
m_{\chi_{1}^{ \pm}}=M_{\widetilde{W}}-\frac{M_{W}^{2}\left(M_{\widetilde{W}}+\mu \sin 2 \beta\right)}{\mu^{2}-M_{\widetilde{W}}^{2}} \\
m_{\chi_{1}^{0}}=M_{\widetilde{B}}+\sin ^{2} \theta_{W} \frac{m_{Z}^{2}\left(M_{\widetilde{B}}+\mu \sin 2 \beta\right)}{\left(M_{\widetilde{B}}^{2}-\mu^{2}\right)} \\
m_{\chi_{2}^{0}}=M_{\widetilde{W}}+\cos ^{2} \theta_{W} \frac{m_{Z}^{2}\left(M_{\widetilde{W}}+\mu \sin 2 \beta\right)}{\left(M_{\widetilde{W}}^{2}-\mu^{2}\right)}
\end{gathered}
$$

where $M_{\tilde{B}}$ and $M_{\widetilde{W}}$ are determined using Equation 2.1, $\theta_{W}\left(\sin ^{2} \theta_{W}=0.234\right)$ is the Weinberg mixing angle, $M_{W}\left(=80.33 \mathrm{GeV} / \mathrm{c}^{2}\right)$ is the $W^{ \pm}$mass and $M_{Z}\left(=91.187 \mathrm{GeV} / \mathrm{c}^{2}\right)$ is the $Z^{0}$ mass. The slepton masses are determined by Equations 2.2 and are functions of the squark and gluino masses and $\tan \beta$. Figures 2.2-2.10 show $\mathrm{M}_{\tilde{\chi}_{1}^{ \pm}}, \mathrm{M}_{\tilde{\chi}_{1}^{0}}$ and $\mathrm{M}_{\tilde{\chi}_{2}^{0}}$ as functions of $\tan \beta, \mu$ and $\mathrm{M}_{\tilde{g}}$. Note that the masses of each particle is primarily a function of $\mathrm{M}_{\tilde{g}}$, and not of either $\tan \beta$ or $\mu$.

The Higgsino and gaugino content of the neutralinos can be determined using [17]:

$$
\left(\begin{array}{c}
\tilde{\chi}_{1}^{0} \\
\tilde{\chi}_{2}^{0} \\
\tilde{\chi}_{3}^{0} \\
\tilde{\chi}_{4}^{0}
\end{array}\right)=\left(\begin{array}{cccc}
1 & 0 & \frac{s_{\theta}\left(s_{\beta}+c_{\beta}\right) m_{Z}}{\sqrt{2}\left(\mu-M_{\widetilde{B}}\right)} & \frac{s_{\theta}\left(s_{\beta}-c_{\beta}\right) m_{Z}}{\sqrt{2}\left(\mu+M_{\widetilde{B}}\right)} \\
0 & 1 & -\frac{c_{\theta}\left(s_{\beta}+c_{\beta}\right) m_{Z}}{\sqrt{2}\left(\mu-M_{\widetilde{W}}\right)} & -\frac{c_{\theta}\left(s_{\beta}-c_{\beta}\right) m_{Z}}{\sqrt{2}\left(\mu+M_{\widetilde{W}}\right)} \\
-\frac{s_{\theta}\left(s_{\beta}+c_{\beta}\right) m_{Z}}{\sqrt{2}\left(\mu-M_{\widetilde{B}}\right)} & \frac{c_{\theta}\left(s_{\beta}+c_{\beta}\right) m_{Z}}{\sqrt{2}\left(\mu-M_{\widetilde{W}}\right)} & 1 & 0 \\
-\frac{s_{\theta}\left(s_{\beta}-c_{\beta}\right) m_{Z}}{\sqrt{2}\left(\mu+M_{\widetilde{B}}\right)} & \frac{c_{\theta}\left(s_{\beta}-c_{\beta}\right) m_{Z}}{\sqrt{2}\left(\mu+M_{\widetilde{W}}\right)} & 0 & 1
\end{array}\right)\left(\begin{array}{c}
\widetilde{B} \\
\widetilde{W} \\
\widetilde{H}_{A} \\
\widetilde{H}_{S}
\end{array}\right)
$$

where $s_{x}\left(c_{x}\right)$ is the sine (cosine) of the appropriate angle. It is immediately obvious that the $\tilde{\chi}_{1}^{0}$ is mainly $\tilde{B}$ with some Higgsino content and the $\tilde{\chi}_{2}^{0}$ is mainly $\tilde{W}_{3}$ with some Higgsino content. Figures 2.11-2.15 show the Higgsino fraction of $\tilde{\chi}_{2}^{0}$ as a function of $\tan \beta, \mu$ and $\mathrm{M}_{\tilde{g}}$. 


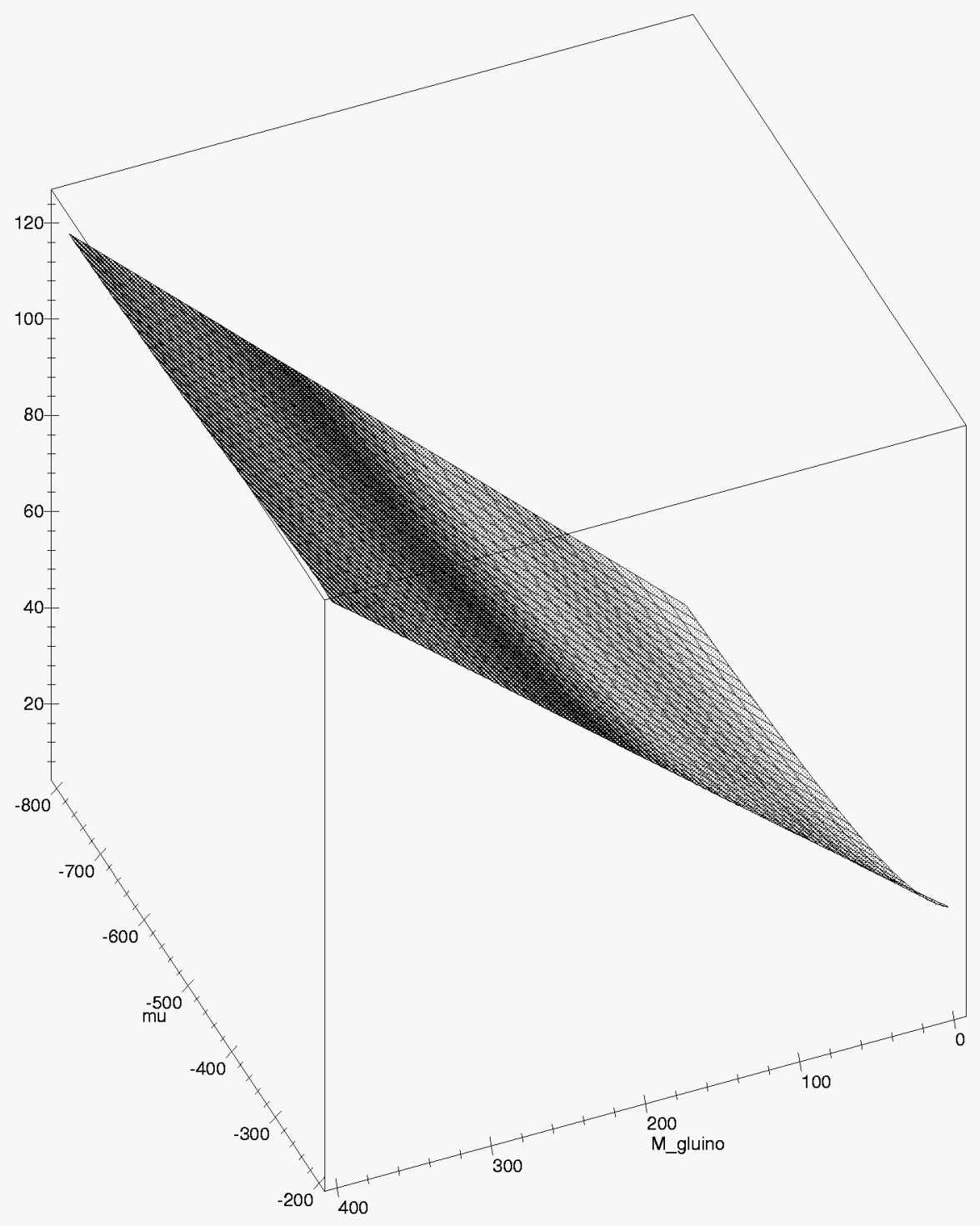

Figure 2.2: $\mathrm{M}_{\tilde{\chi}_{1}^{ \pm}}$(vertical axis; in $\mathrm{GeV} / \mathrm{c}^{2}$ ) as a function of $\mu$ (left axis; in $\mathrm{GeV} / \mathrm{c}^{2}$ ) and $\mathrm{M}_{\tilde{g}}$ (right axis; in $\left.\mathrm{GeV} / \mathrm{c}^{2}\right)$ for $\tan \beta=2$. Note that the $\tilde{\chi}_{1}^{ \pm}$mass has little $\mu$ dependence. The shading in this and the following figures is an artifact of the program used to generate the plots. 


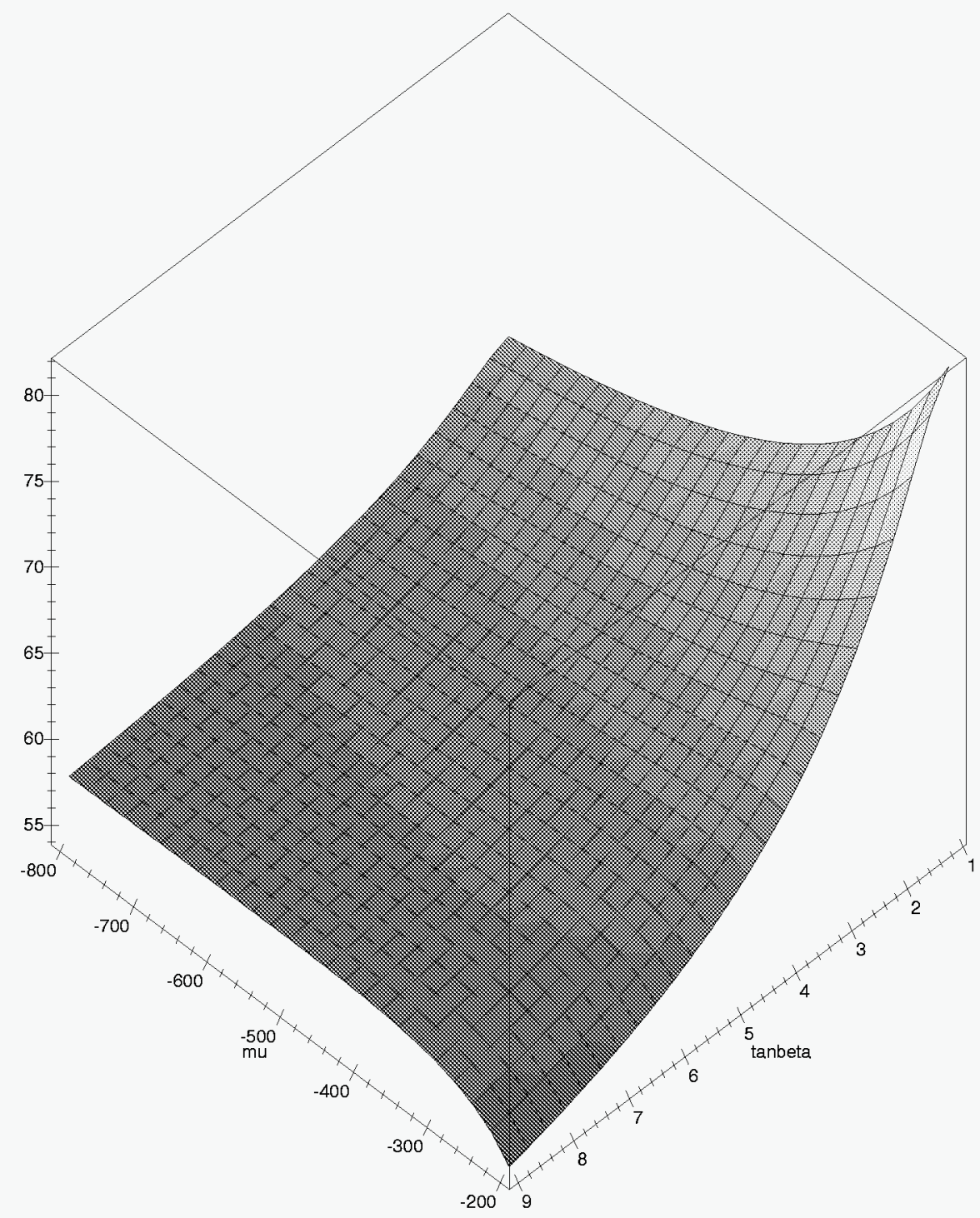

Figure 2.3: $\mathrm{M}_{\tilde{\chi}_{1}^{ \pm}}$(vertical axis; in $\mathrm{GeV} / \mathrm{c}^{2}$ ) as a function of $\mu$ (left axis; in $\mathrm{GeV} / \mathrm{c}^{2}$ ) and $\tan \beta$ (right axis) for $\mathrm{M}_{\tilde{g}}=200 \mathrm{GeV} / \mathrm{c}^{2}$. Note that the vertical axis has a range of only $15 \mathrm{GeV} / \mathrm{c}^{2}$; the $\tilde{\chi}_{1}^{ \pm}$mass has much smaller dependence on $\mu$ and $\tan \beta$ than on $\mathrm{M}_{\tilde{g}}$. 


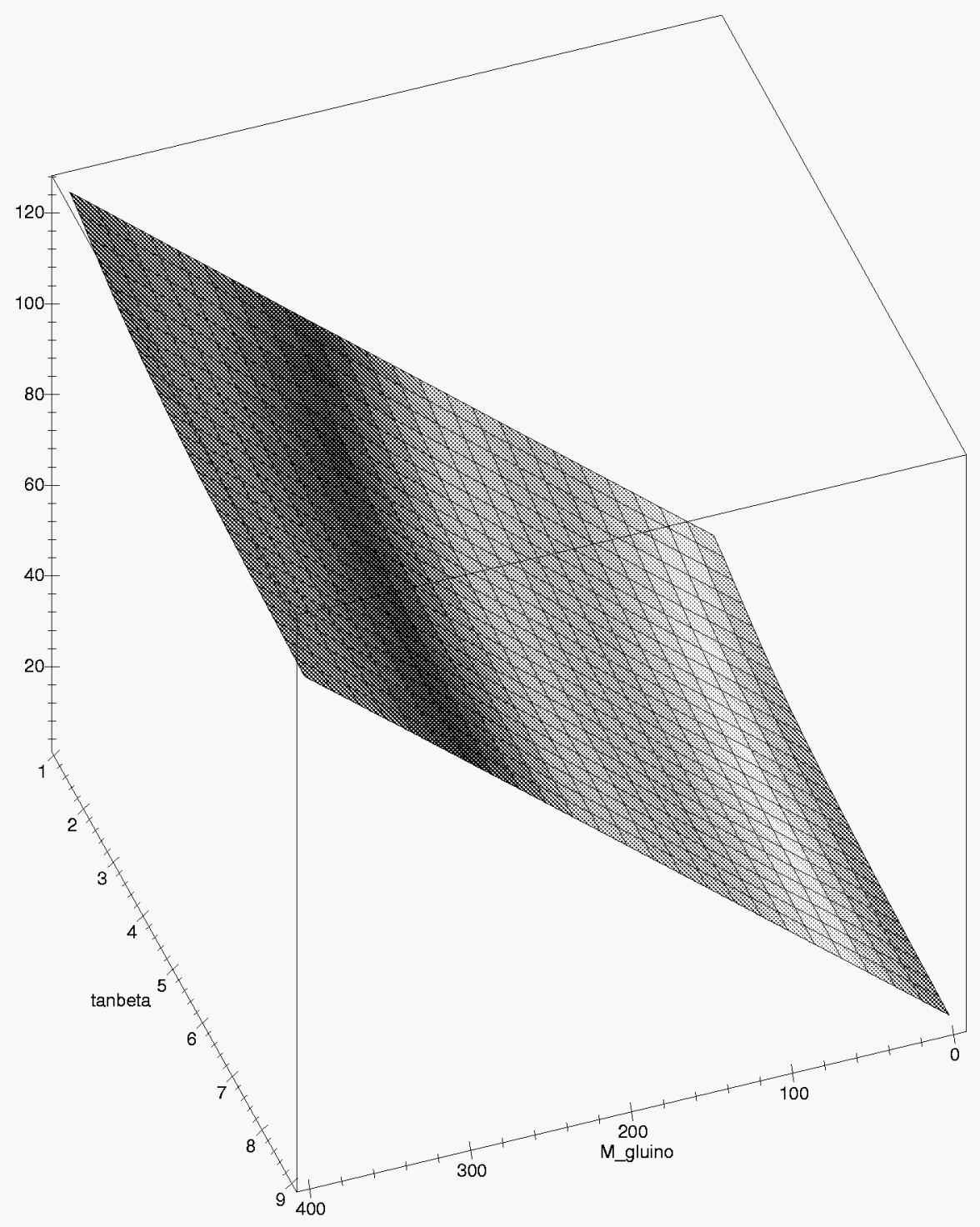

Figure 2.4: $\mathrm{M}_{\tilde{\chi}_{1}^{ \pm}}$(vertical axis; in $\mathrm{GeV} / \mathrm{c}^{2}$ ) as a function of $\tan \beta$ (left axis) and $\mathrm{M}_{\tilde{g}}$ (right axis; in $\mathrm{GeV} / \mathrm{c}^{2}$ ) for $\mu=-400 \mathrm{GeV} / \mathrm{c}^{2}$. Note that the mass has little $\tan \beta$ dependence. 


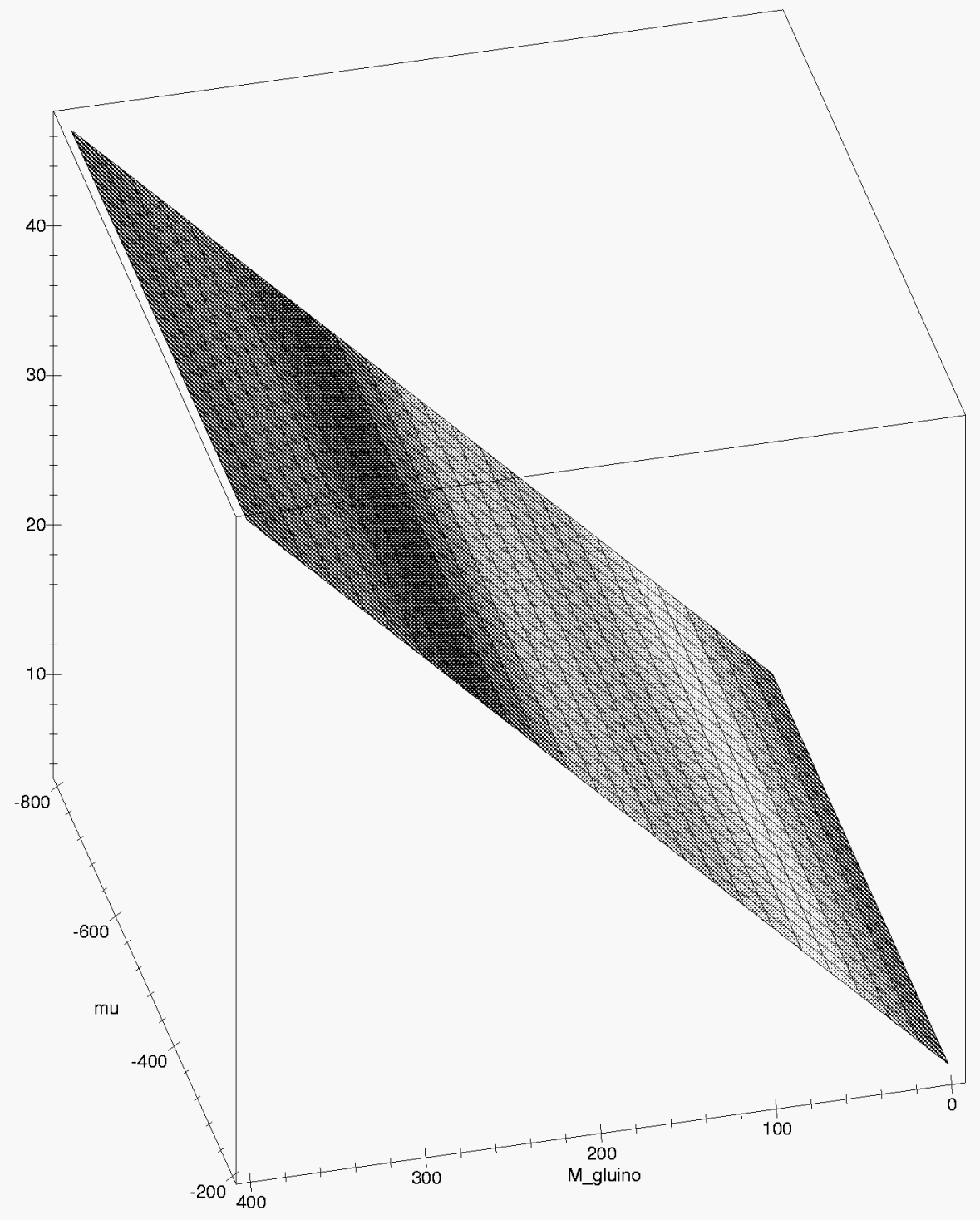

Figure 2.5: $\mathrm{M}_{\tilde{\chi}_{1}^{0}}$ (vertical axis; in $\mathrm{GeV} / \mathrm{c}^{2}$ ) as a function of $\mu$ (left axis; in $\mathrm{GeV} / \mathrm{c}^{2}$ ) and $\mathrm{M}_{\tilde{g}}$ (right axis; in $\mathrm{GeV} / \mathrm{c}^{2}$ ) for $\tan \beta=2$. Note that the mass is largely independent of $\mu$. 


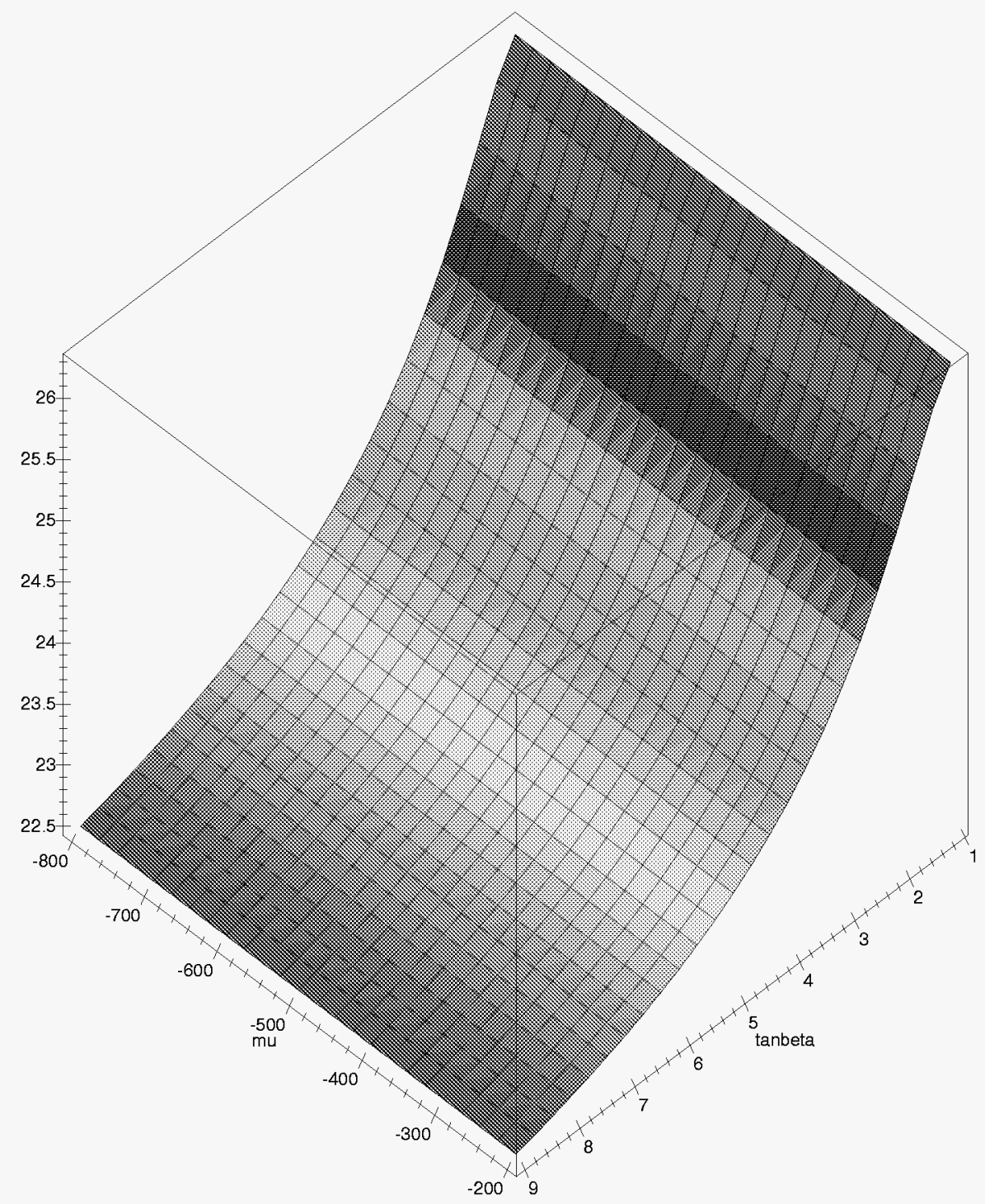

Figure 2.6: $\mathrm{M}_{\tilde{\chi}_{1}^{0}}$ (vertical axis; in $\mathrm{GeV} / \mathrm{c}^{2}$ ) as a function of $\mu$ (left axis; in $\mathrm{GeV} / \mathrm{c}^{2}$ ) and $\tan \beta$ (right axis) for $\mathrm{M}_{\tilde{g}}=200 \mathrm{GeV} / \mathrm{c}^{2}$. Note that the mass is independent of $\mu$ and only weakly depends on $\tan \beta$ (the vertical scale only spans $4 \mathrm{GeV} / \mathrm{c}^{2}$ ). 


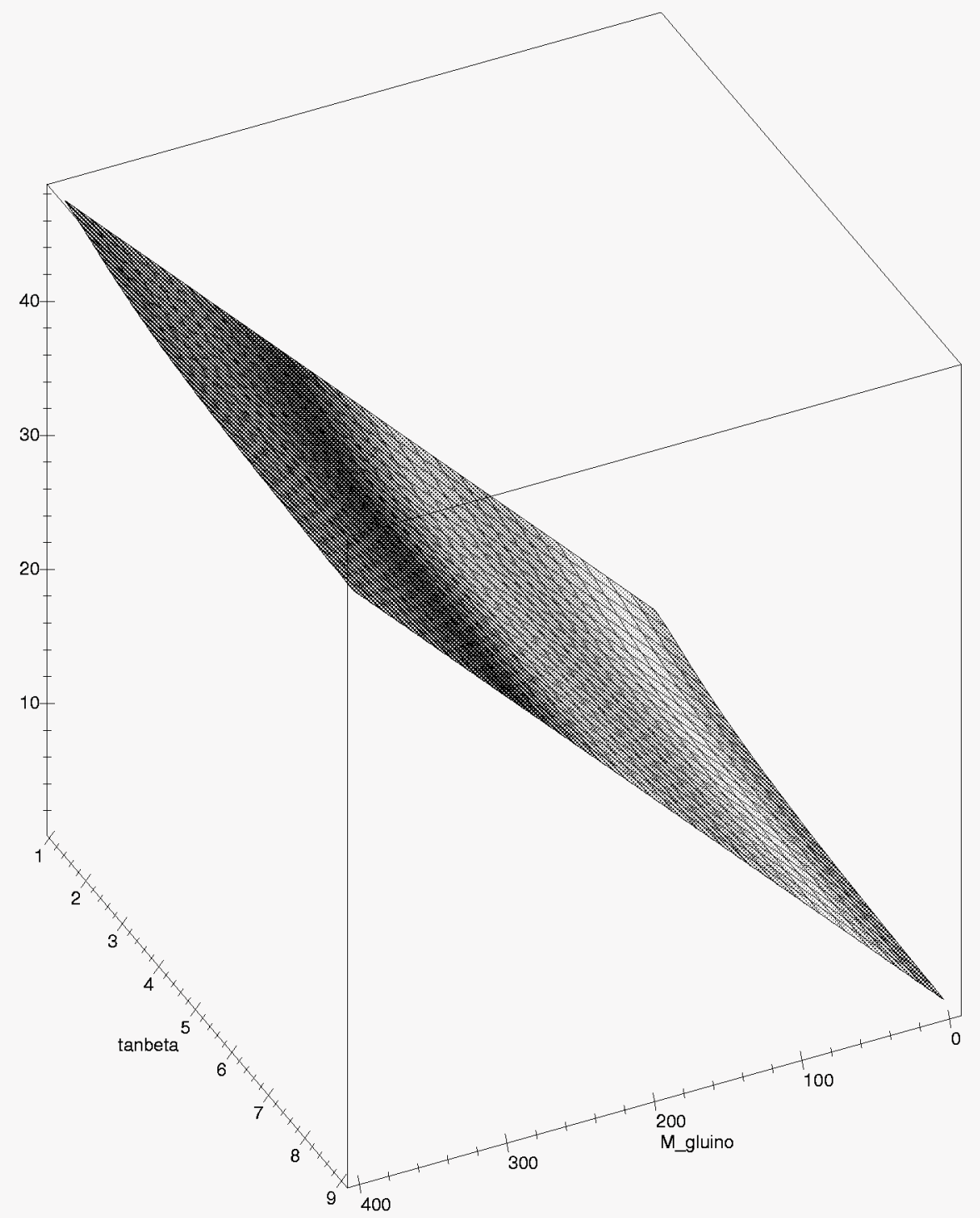

Figure 2.7: $\mathrm{M}_{\tilde{\chi}_{1}^{0}}$ (vertical axis; in $\mathrm{GeV} / \mathrm{c}^{2}$ ) as a function of $\tan \beta$ (left axis) and $\mathrm{M}_{\tilde{g}}$ (right axis; in $\mathrm{GeV} / \mathrm{c}^{2}$ ) for $\mu=-400 \mathrm{GeV} / \mathrm{c}^{2}$. 


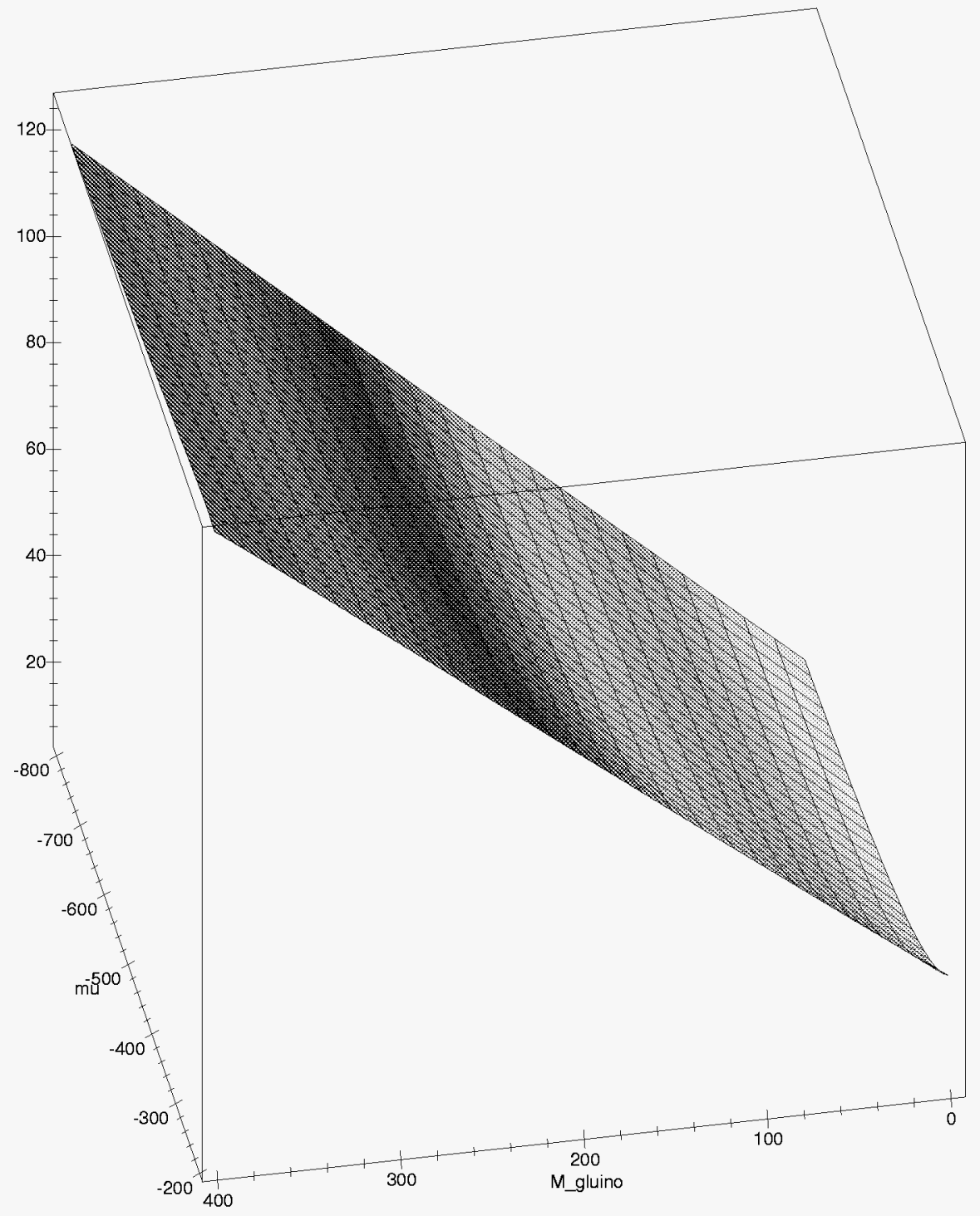

Figure 2.8: $\mathrm{M}_{\tilde{\chi}_{2}^{0}}$ (vertical axis; in $\mathrm{GeV} / \mathrm{c}^{2}$ ) as a function of $\mu$ (left axis; in $\mathrm{GeV} / \mathrm{c}^{2}$ ) and $\mathrm{M}_{\tilde{g}}$ (right axis; in $\mathrm{GeV} / \mathrm{c}^{2}$ ) for $\tan \beta=2$. 


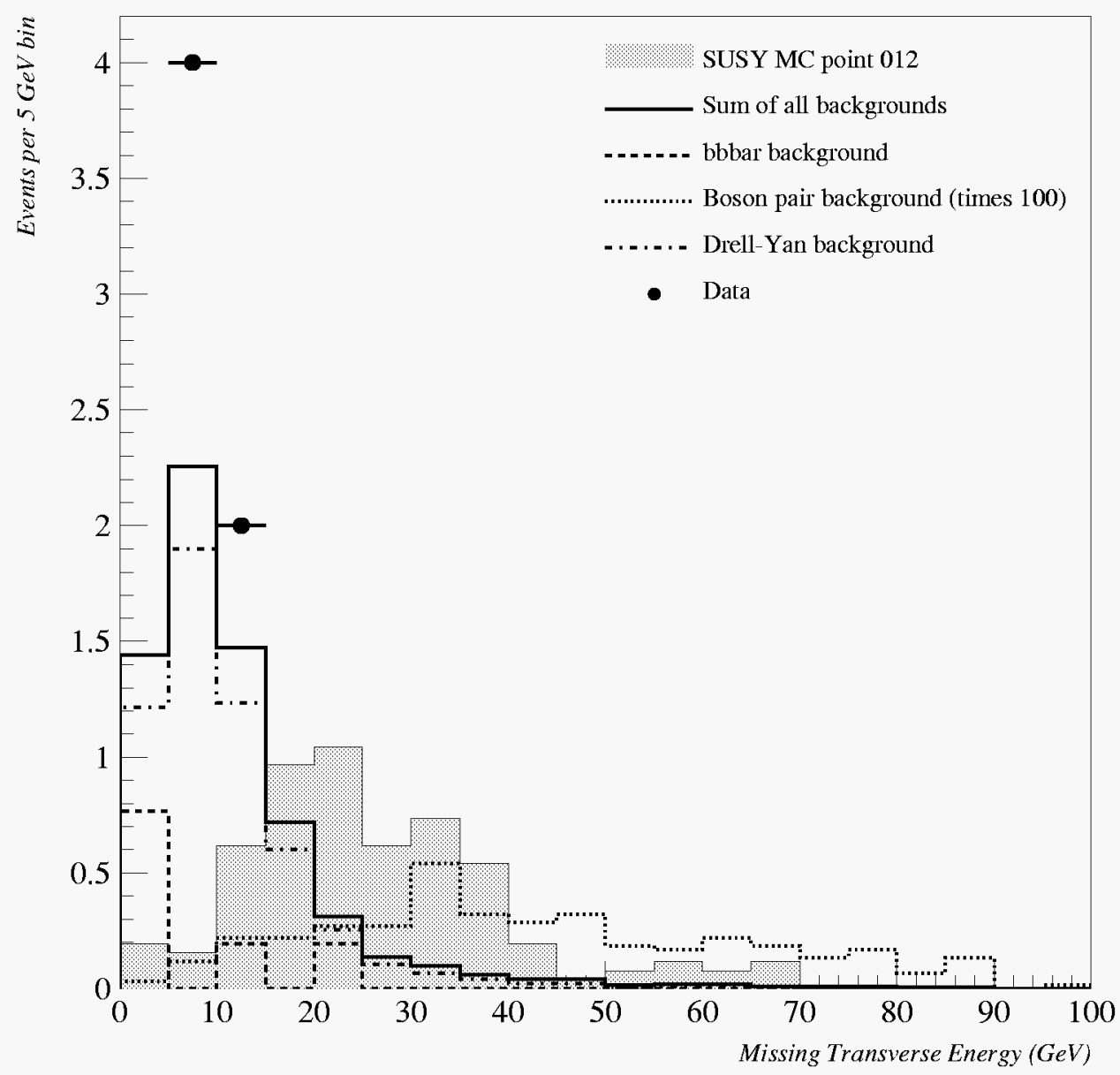

Figure 4.28: Missing transverse energy for each of the types of background and a sample signal point (012). The dashed line is $b \bar{b}$, the dot-dash line is Drell-Yan, the dotted line is boson pair production and the solid line is the sum. The filled histogram is MC signal point 012. Each histogram is normalized such that the area of the histogram is equal to the number of events expected after all cuts but before the $\not_{T}$ cut. The boson pair histogram is multiplied by 100 so it is visible. The data are indicated by circles $(\bullet)$. 
Table 4.9: Number of expected background events after each cut, for contributions from $b \bar{b} / c \bar{c}$, boson pair, Drell-Yan and $t \bar{t}$. All numbers are scaled by the Monte Carlo correction factor discussed in Chapter 3. The fake lepton rate (0.00289/event) is applied to all dilepton backgrounds. As can be seen, the isolation and $\Delta R$ cuts reduce the heavy flavor contribution, the $Z^{0}$ mass cut removes the boson pair and Drell-Yan contributions, and the $\Delta \phi_{\ell \ell}$ cut further reduces the Drell-Yan contribution. The $E_{T}$ cut reduces the total expected background to 0.99 events.

\begin{tabular}{|lrrrr|r|}
\hline & \multicolumn{5}{c|}{ Events remaining after each cut } \\
Cut & $b \bar{b} / c \bar{c}$ & Boson pair & Drell-Yan & $t \bar{t}$ & Sum \\
\hline 3 lepton requirement & 14.83 & 0.58 & 20.46 & 0.05 & 35.92 \\
good run requirement & 14.83 & 0.58 & 20.46 & 0.05 & 35.92 \\
cosmic removal & 14.83 & 0.58 & 20.46 & 0.05 & 35.92 \\
$\left|z_{\text {vertex }}\right|<60 \mathrm{~cm}$ & 14.60 & 0.52 & 19.57 & 0.04 & 34.73 \\
Vertex Requirements & 14.22 & 0.52 & 19.57 & 0.04 & 34.35 \\
$I S O<2$ & 3.17 & 0.48 & 18.77 & 0.02 & 22.44 \\
$\left|\mathrm{Q}_{1}+\mathrm{Q}_{2}+\mathrm{Q}_{3}\right|<3$ & 3.17 & 0.48 & 18.77 & 0.02 & 22.44 \\
Require $e^{+} e^{-}$or $\mu^{+} \mu^{-}$ & 2.71 & 0.46 & 18.30 & 0.02 & 21.49 \\
$\Delta R_{\ell \ell}>0.4$ & 1.70 & 0.45 & 18.21 & 0.01 & 20.37 \\
$\Delta \phi_{\ell_{1} \ell_{2}}<170^{\circ}$ & 1.70 & 0.38 & 7.86 & 0.00 & 9.94 \\
$Z^{0}$ removal $\left(75-105 \mathrm{GeV} / \mathrm{c}^{2}\right)$ & 1.70 & 0.04 & 5.81 & 0.00 & 7.55 \\
$\Upsilon$ removal $\left(9-11 \mathrm{GeV} / \mathrm{c}^{2}\right)$ & 1.70 & 0.04 & 5.49 & 0.00 & 7.23 \\
$\mathrm{~J} / \psi$ removal $\left(2.9-3.1 \mathrm{GeV} / \mathrm{c}^{2}\right)$ & 1.15 & 0.04 & 5.49 & 0.00 & 6.68 \\
$\not_{T}>15 \mathrm{GeV}$ & 0.00 & 0.02 & 0.97 & 0.00 & 0.99 \\
\hline
\end{tabular}

\subsubsection{Estimate of Expected Background}

We generate a large quantity of Monte Carlo background for each type of event: at least $60 \mathrm{pb}^{-1} \mathrm{for}$ $b \bar{b} / c \bar{c}, 100-400 \mathrm{pb}^{-1}$ for each of the Drell-Yan types, $5,000 \mathrm{pb}^{-1}$ for $t \bar{t}$ and $2,000-7,000 \mathrm{pb}^{-1}$ for boson pair. We examine each type of background with our analysis code and apply the lepton ID cuts and additional cuts described above. Table 4.9 lists the number of events expected after each cut from each type of background. Table 4.10 compares the sum from background to the number of data events and a sample MC signal point. As can be seen, the data and the MC background agree quite well. After all cuts, we expect to see $0.99 \pm 0.2$ events and see none.

After all cuts, no events remain in the data and the number of events before the final cuts are applied agree well with the expected background. We see no evidence for Supersymmetry. 
Table 4.10: Number of expected background, data and signal Monte Carlo events left after each cut. Below the line the data and background predictions agree quite well. Above the line the cuts remove both predictable background and unpredictable background (such as cosmic rays and multiple interactions). This is especially true for the $\Delta R$ cut- we expect to remove 1 event and instead remove 14. Examination of these events shows they are clearly cosmic rays. After all cuts, we expect 0.99 events from background and see none. For the sample Monte Carlo signal point 012, we expect 4.47 events after all cuts.

\begin{tabular}{|lrr|c|}
\hline Cut & Background & Data & Signal MC \\
\hline 3 lepton requirement & 35.92 & 247 & 8.44 \\
good run requirement & 35.92 & 232 & 8.44 \\
cosmic removal & 35.92 & 70 & 8.44 \\
$\left|z_{\text {vertex }}\right|<60 \mathrm{~cm}$ & 34.73 & 66 & 8.01 \\
Vertex Requirements & 34.35 & 59 & 8.01 \\
$I S O<2$ & 22.44 & 23 & 6.89 \\
$\left|\mathrm{Q}_{1}+\mathrm{Q}_{2}+\mathrm{Q}_{3}\right|<3$ & 22.44 & 23 & 6.89 \\
Require $e^{+} e^{-}$or $\mu^{+} \mu^{-}$ & 21.49 & 23 & 6.89 \\
$\Delta R_{\ell \ell}>0.4$ & 20.37 & 9 & 6.81 \\
\hline$\Delta \phi_{\ell_{1} \ell_{2}}<170^{\circ}$ & 9.94 & 8 & 6.19 \\
$Z^{0}$ removal $\left(75-105 \mathrm{GeV} / \mathrm{c}^{2}\right)$ & 7.55 & 7 & 5.95 \\
$\Upsilon$ removal $\left(9-11 \mathrm{GeV} / \mathrm{c}^{2}\right)$ & 7.23 & 7 & 5.49 \\
$\mathrm{~J} / \psi$ removal $\left(2.9-3.1 \mathrm{GeV} / \mathrm{c}^{2}\right)$ & 6.68 & 6 & 5.49 \\
$\mathscr{E}_{T}>15 \mathrm{GeV}$ & 0.99 & 0 & 4.47 \\
\hline
\end{tabular}




\section{Chapter 5}

\section{Limits on the SiMSSM parameter space}

We see no evidence for Supersymmetry. However, it is instructive to examine a few models and set limits on the production of $\tilde{\chi}_{1}^{ \pm}$and $\tilde{\chi}_{2}^{0}$. We can then find the naturalness of SUSY with our limits, and can also compare our sensitivity to that of other experiments. I will describe the Monte Carlo simulation techniques, the limit setting method, and the limits on the SUGRA-inspired MSSM, the $\mathrm{SU}(5) \times \mathrm{U}(1)$ Supergravity model, and SUGRA.

\subsection{Monte Carlo Simulation techniques}

Simulating both background and SUSY signal events is a two stage process. First, we use a Monte Carlo program to generate the events. For this analysis, we use ISAJET v7.06 (for much of the background calculation) and ISAJET v7.20 (for all of the signal calculation) [36]. There are no substantial differences between the versions; v7.20 contains bug fixes which were corrected manually in v7.06. ISAJET is a general Monte Carlo program that can be used to generate events for any Standard Model process as well as many different SM extensions. We use it for all the background processes (see Section 4.6) and to generate a wide variety of points in SiMSSM space.

A generated event cannot be examined using the analysis code. The information is limited to the $P_{T}, \eta$ and $\phi$ for each particle and the decay chains. We use QFL (see Section 3.2.7) to include detector effects (such as resolution and smearing). As mentioned in Section 3.2.7, QFL is over-efficient for lepton identification so we apply an overall correction factor of $0.864 /$ event. After the events generated with ISAJET are simulated with QFL, they are identical in structure to data taken with the detector.

The simulated events are examined with the analysis program, with one addition. Data that is recorded must pass through the trigger system. QFL simulation does not include a simulation of 
the trigger. We use a trigger simulation program written specifically for SUSY analyses, MC_WGT.

\subsubsection{Trigger Simulation}

There is no specific trigger path which events must follow to enter our data set. This has both advantages and disadvantages. Since this is a search for new physics, allowing all possible signal events into the data is the proper technique. However, this means events can follow any one of many trigger paths. For the dilepton data set on which this analysis is based, many different triggers contribute to the data set. To properly simulate a trigger, we must include several effects: the actual trigger efficiency and both static and dynamic prescales.

The trigger efficiency for each trigger is taken from a variety of CDF Notes [38]. This information is coded (in either $500 \mathrm{MeV}$ or $1 \mathrm{GeV}$ lepton $E_{T}\left(P_{T}\right)$ bins) into the trigger simulation routine MC_WGT. Each event is passed to this routine. The $E_{T}\left(P_{T}\right)$ and $\eta$ for each lepton in the event is examined to see which possible Level 1, Level 2 and Level 3 triggers it could have passed. For many detector regions, there are several possible triggers which could be used. The trigger which returns the highest probability at each level is the probability that that lepton would pass that trigger level. The values for different detector regions and lepton species are ORed together ${ }^{1}$ and this number is the probability that this event would pass that trigger level. The values returned from each trigger level are multiplied and the total is the probability that this particular event would have entered the data set.

To calculate the static and dynamic prescales, we use SIGMON [39]. SIGMON examines the online LUMMON ${ }^{2}$ data files to find the actual luminosity recorded for each trigger on a run-by-run basis. This can be compared to the luminosity recorded for each run to determine the effective prescale of each trigger for each run. We divide the Run IB data into three bins according to the average instantaneous luminosity for each run $\left(\mathcal{L}<1.7 \times 10^{30} \mathrm{~s}^{-1} \mathrm{~cm}^{-2}, 1.7<\mathcal{L}<4 \times 10^{30} \mathrm{~s}^{-1} \mathrm{~cm}^{-2}\right.$ and $\mathcal{L}>4 \times 10^{30} \mathrm{~s}^{-1} \mathrm{~cm}^{-2}$ ) and find the effective prescale for each trigger in each $\mathcal{L}$ bin (Table $5.1^{3}$ ). We then calculate a weighted average of each of the prescale bins as a function of the percent of the total integrated luminosity from Run IB in each bin. The low instantaneous $\mathcal{L}$ bin has $43 \%$

${ }^{1}$ If trigger $a$ has efficiency $\epsilon_{a}$ and trigger $b$ has efficiency $\epsilon_{b}$ the total efficiency is

$$
\epsilon_{a+b}=\epsilon_{a}+\epsilon_{b}\left(1-\epsilon_{a}\right) .
$$

${ }^{2}$ LUMMON is an online program that monitors the amount of data recorded by each trigger during a run. It also controls the dynamic prescales.

${ }^{3}$ The names of the triggers can easily be converted to a meaningful statement. For example, the trigger CEM_8_CFT_7_5_XCES requires at least $8 \mathrm{GeV}$ in the CEM, a CFT track with $P_{T}$ above $7.5 \mathrm{GeV} / \mathrm{c}$ and a good hit in the CES. Numbers written as words ('TWO') indicate the number of objects required in a particular detector. 5DEG indicates how close a muon stub must be to matching the extrapolation of a CTC track. 
Table 5.1: The Level 2 triggers used in this analysis and the effective prescale as a function of instantaneous luminosity. For this analysis, low $\mathcal{L}$ means $\mathcal{L}<1.7 \times 10^{30} \mathrm{~s}^{-1} \mathrm{~cm}^{-2}$ (43\% of the data), middle is $1.7<\mathcal{L}<4 \times 10^{30} \mathrm{~s}^{-1} \mathrm{~cm}^{-2}$ (41\% of the data) and high is $\mathcal{L}>4 \times 10^{30} \mathrm{~s}^{-1} \mathrm{~cm}^{-2}(16 \%$ of the data).

\begin{tabular}{|l|rrr|r|}
\hline Trigger & Low $\mathcal{L}$ & Middle $\mathcal{L}$ & High $\mathcal{L}$ & Average \\
\hline CEM_8_CFT_7_5_XCES & 1.11 & 1.29 & 1.55 & 1.21 \\
TWO_CMU_TWO_CFT_2_2 & 1.00 & 1.00 & 1.00 & 1.00 \\
CMUP_CFT_12_5DEG & 1.00 & 1.00 & 1.00 & 1.00 \\
CEM_5_CFT_4_7_CMU_2_7 & 1.72 & 2.19 & 3.35 & 2.14 \\
CMX_CMU_TWO_CFT_2_2 & 1.05 & 1.39 & 3.02 & 1.50 \\
TWO_CMU_ONE_CFT_2_2_6TOW & 1.11 & 1.26 & 1.56 & 1.24 \\
CMNP_CFT_12_5DEG & 1.35 & 7.58 & 31.27 & 8.69 \\
CMUP_CFT_7_5_5DEG & 1.18 & 2.52 & 6.16 & 2.53 \\
CEM_16_CFT_12 & 1.00 & 1.00 & 1.00 & 1.00 \\
CMX_CFT_12_5DEG & 5.25 & 5.70 & 16.67 & 7.26 \\
\hline
\end{tabular}

of the data, the middle instantaneous $\mathcal{L}$ bin $41 \%$, and the high instantaneous $\mathcal{L}$ bin $16 \%$. This final number is the effective prescale for the entire IB data set for each trigger. It is entered in the MC_WGT routine and applied to each event, depending on which trigger that event passes.

This technique for including the prescale effect as a part of the trigger efficiency simplifies several calculations. This also automatically applies the prescale to both background and signal Monte Carlo studies.

To minimize the statistical uncertainty in our Monte Carlo studies, we generate numbers of events corresponding to large $\int \mathcal{L} d t$. We maintain a database listing the $\int \mathcal{L} d t$ for each sample and use this normalize to the amount of data present in Run IB. This constant is also included as part of the trigger efficiency.

The final trigger efficiency returned for each event is a product of four numbers: a weighting factor for the $\int \mathcal{L} d t$, the Level 1 trigger efficiency, the Level 2 trigger efficiency (including the prescale) and the Level 3 trigger efficiency.

\section{$5.2 \quad$ Limit setting method}

We generate and analyze a statistically significant number of events for a particular point in SiMSSM space to determine if we should see evidence for $\tilde{\chi}_{1}^{ \pm} \tilde{\chi}_{2}^{0}$ production at that point. We use the formula 


$$
\sigma_{\tilde{\chi}_{1}^{ \pm} \tilde{\chi}_{2}^{0}} \cdot \operatorname{BR}\left(\tilde{\chi}_{1}^{ \pm} \tilde{\chi}_{2}^{0} \rightarrow 3 \ell+X\right)>\frac{N_{e x p}}{\epsilon^{t o t} \cdot \int \mathcal{L} d t}
$$

to compare the predicted production cross section $\left(\sigma_{\tilde{\chi}_{1}^{ \pm} \tilde{\chi}_{2}^{0}}\right)$ times branching ratio to trileptons $\left(\mathrm{BR}\left(\tilde{\chi}_{1}^{ \pm} \tilde{\chi}_{2}^{0} \rightarrow 3 \ell+X\right)\right)$ with the number of expected events $\left(\mathrm{N}_{\text {exp }}\right)$ divided by the total event acceptance $\left(\epsilon^{t o t}\right)$ times the integrated luminosity.

\subsection{1 $\mathrm{N}_{\text {exp }}$ : Systematic Uncertainty}

The number of expected events is calculated by taking a Poisson distribution for having seen 0 events and convolving it with a Gaussian ${ }^{4}$ whose width is determined by the total systematic uncertainty. The systematic uncertainty has several components:

- Uncertainty in the total integrated luminosity: $8 \%$ [40].

- Uncertainty in the trigger efficiency [41]. We determine the trigger efficiency for a particular trigger by studying electron or muon events which pass triggers other than the one we are examining. We then impose the trigger conditions and count the number of events passing. We find that the total trigger efficiencies for electrons and muons are [42, 43]

$$
\begin{aligned}
\epsilon_{e}^{\text {trig }} & =\left(87.3_{-4.9}^{+3.8}\right) \% \\
\epsilon_{\mu}^{\text {trig }} & =(87.1 \pm 2.9) \%
\end{aligned}
$$

In a multi-lepton event analysis, we must take into account the possibility of events with multiple trigger-leptons: (a) one trigger lepton and (b) two trigger leptons. [Note that there were no three lepton triggers available in Run IB, so we do not have to worry about that case.] We obtain a conservative estimate of the trigger efficiency uncertainty by assuming all dilepton events are in case (a). The electron trigger has the largest uncertainty and so we use that value. This situation gives a trigger efficiency uncertainty of $\pm 5.6 \%$.

- Uncertainty in the trilepton finding efficiency:

\footnotetext{
${ }^{4}$ The number of expected events for having seen 0 using a Poisson distribution at a $95 \%$ Confidence Level is 3.0 . A Poisson distribution can only take integer values. However, we can be more precise if we combine the information from the Poisson distribution with a Gaussian distribution. A Gaussian distribution is continuous. For more details see pg. 166 of Ref. [5].
} 
- Uncertainty in the degradation of the Isolation cut: $\pm 8 \%$ [44]. This is important as we combine Run IA and IB data sets to increase the integrated luminosity (see Section 5.2.3). Run IB had much higher instantaneous luminosity, which degraded the efficiency of the isolation cut.

- Uncertainty in the Monte Carlo correction factor from Section 3.2.7: $\pm 2 \%$. We calculate this by taking the combination of three leptons (one tight and two loose) that give us the largest correction factor using the information in Table 3.2. For this analysis that combination is a tight CEM electron and two loose CMX muons $(0.864 \pm 0.017$ : $0.017 / 0.864=2 \%$ )

- Uncertainty in the trilepton finding efficiency: $\pm 2 \%$. This is calculated by taking the combination of lepton finding efficiencies from Table 3.2 that results in the largest uncertainty. For this analysis that combination is a tight CEM electron and two loose CMIO muons.

- Uncertainty in the structure functions: $\pm 7 \%$. We used CTEQ-3L for all of the signal Monte Carlo generation and compared the results from it and an average of CTEQ-3L, GRV-94lo and MRS-D0'. The largest deviation between the two is our systematic uncertainty.

- Uncertainty in the fit for the detection efficiency (see Section 5.2.2): $\pm 13 \%$.

The uncertainties combine in quadrature for a total systematic uncertainty of $\pm 20 \%$. This yields $N_{e x p}=3.2$ at a Confidence Level of $95 \%$.

\subsection{2 $\epsilon^{t o t}$ : Detection Efficiency}

The detection efficiency is a combination of geometric acceptance, trigger efficiency and kinematic acceptance. Naïvely, one would expect the detection efficiency to be mainly a function of the $\tilde{\chi}_{1}^{ \pm}$, $\tilde{\chi}_{1}^{0}$ and $\tilde{\chi}_{2}^{0}$ masses: since $\mathrm{M}_{\tilde{\chi}_{1}^{ \pm}} \approx \mathrm{M}_{\tilde{\chi}_{2}^{0}} \approx 2 \mathrm{M}_{\tilde{\chi}_{1}^{0}}$, higher $\tilde{\chi}_{1}^{ \pm}$and $\tilde{\chi}_{2}^{0}$ masses result in stiffer (more energetic), easier to detect leptons. Figure 5.1 shows the detection efficiency as a function of $\mathrm{M}_{\tilde{\chi}_{1}^{ \pm}}$ for $\mu=-200 \mathrm{GeV} / \mathrm{c}^{2}, \tan \beta=2$ and $\mathrm{M}_{\tilde{q}}=\mathrm{M}_{\tilde{g}}$. The detection efficiency clearly rises as a function of $\mathrm{M}_{\tilde{\chi}_{1}^{ \pm}}$. Figure 5.2 shows the detection efficiency for a variety of points in SiMSSM space where we varied $\mu, \mathrm{M}_{\tilde{q}} / \mathrm{M}_{\tilde{g}}$ and $\tan \beta$. We fit a straight line to these points to get a function for the detection efficiency as a function of $\mathrm{M}_{\tilde{\chi}_{1}^{ \pm}}$. Figure 5.3 is a comparison between the fit to all points and a variety of subsets.

We found that the detection efficiency was not completely model independent: changing $\tan \beta$ changes the acceptance. Figure 5.4 shows the fit for points where we constrained $\tan \beta$ to be 2. The quality of the fit clearly improves. Because of this, we use the fit for SiMSSM points where $\tan \beta=2$ and the individual acceptances for other points. 


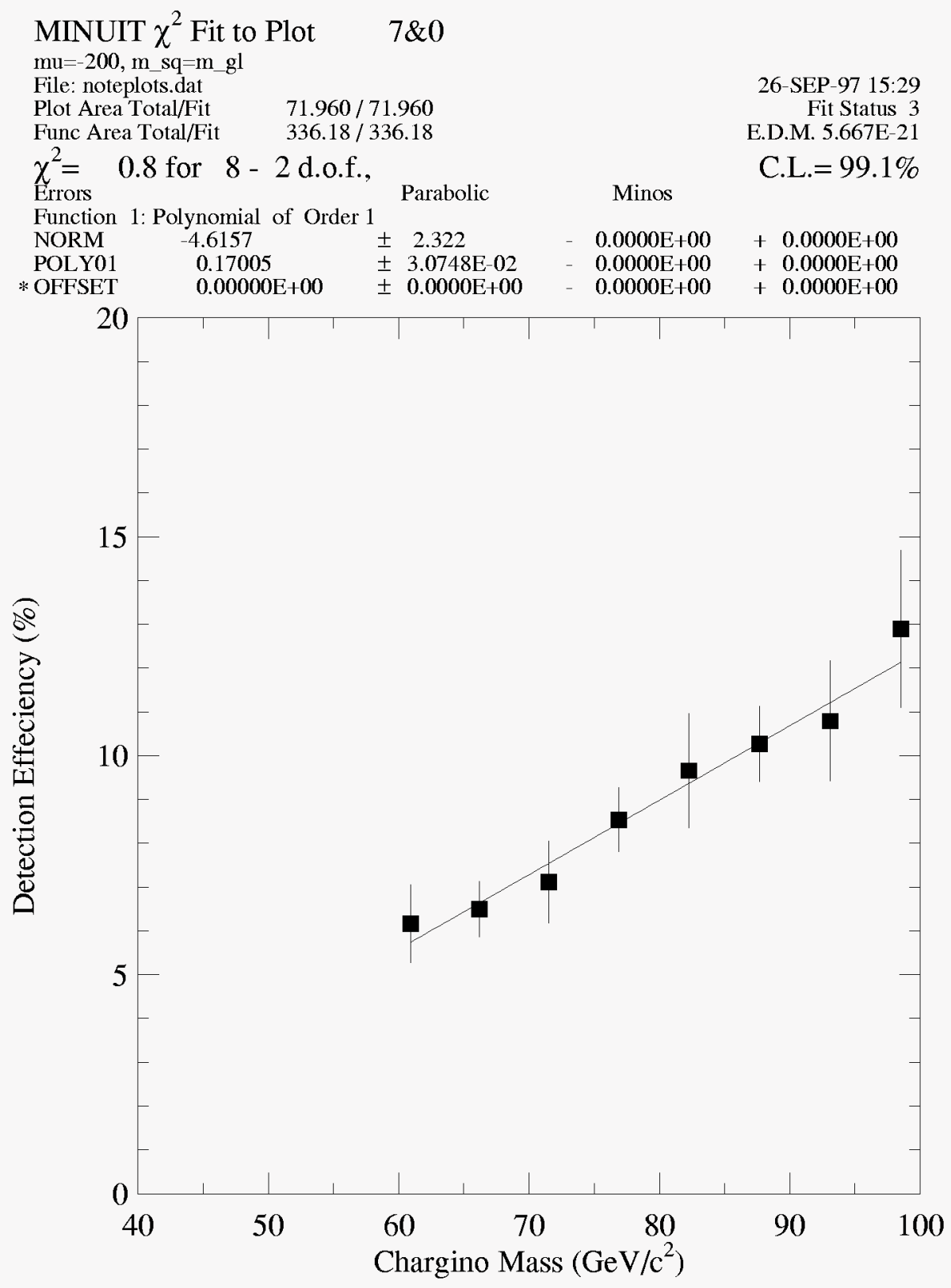

Figure 5.1: The detection efficiency as a function of $\mathrm{M}_{\tilde{\chi}_{1}^{ \pm}}$. For this plot we set $\mu=-200 \mathrm{GeV} / \mathrm{c}^{2}$, $\tan \beta=2$ and $\mathrm{M}_{\tilde{q}}=\mathrm{M}_{\tilde{g}}$. The detection efficiency is clearly a function of the chargino mass. 


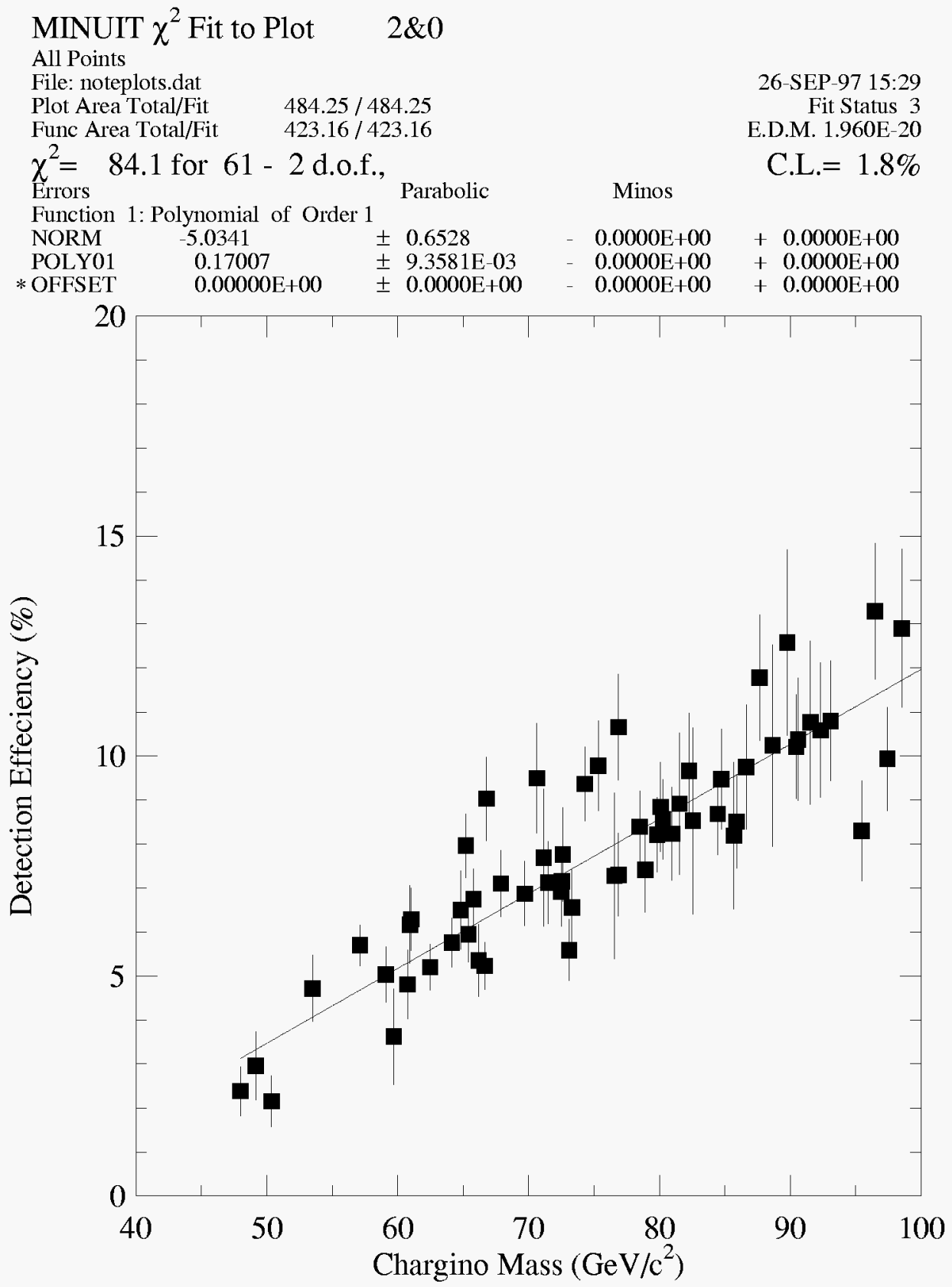

Figure 5.2: The detection efficiency as a function of $\mathrm{M}_{\tilde{\chi}_{1}^{ \pm}}$for a variety of points in SiMSSM space. 


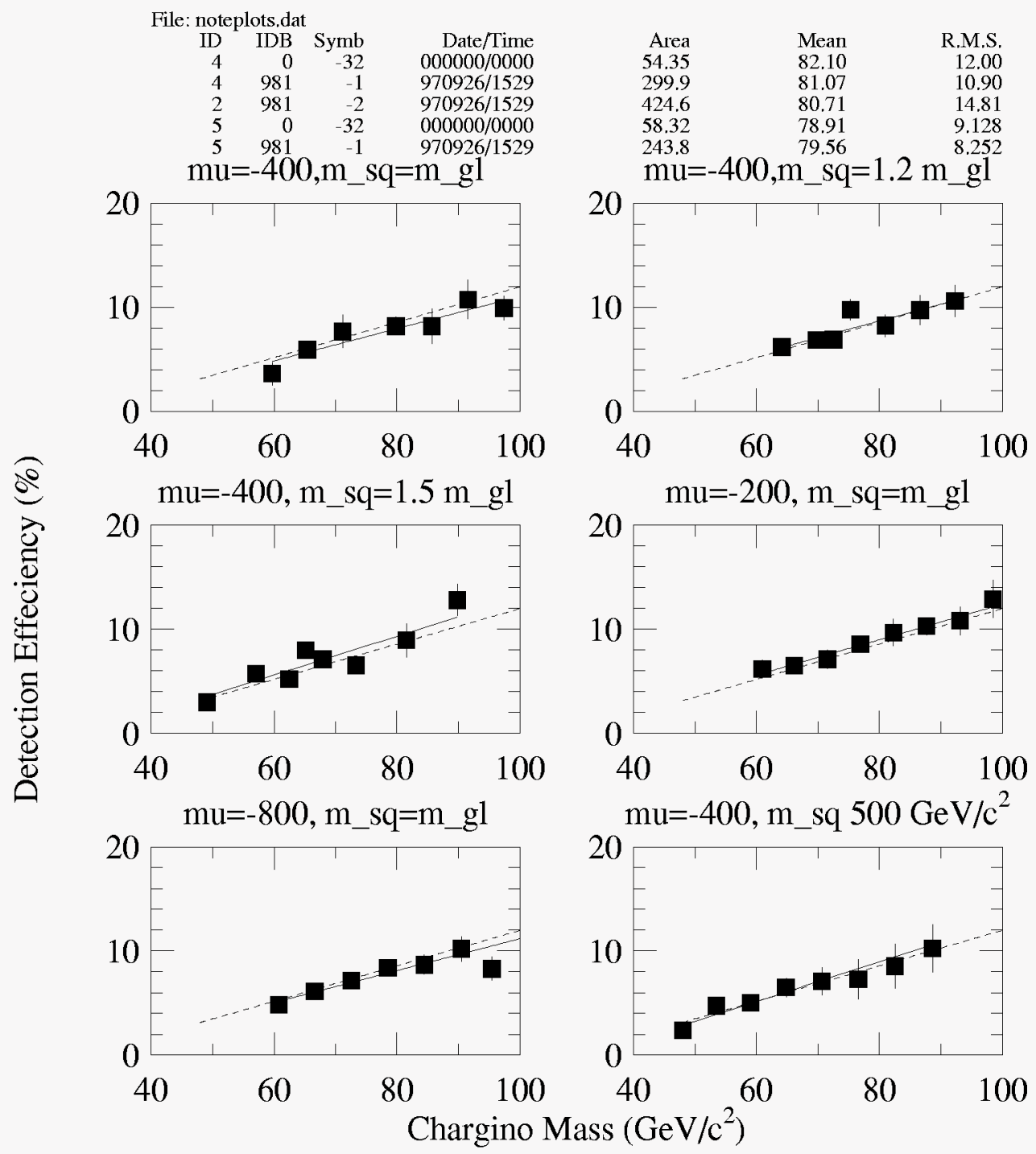

Figure 5.3: The detection efficiency as a function of $\mathrm{M}_{\tilde{\chi}_{1}^{ \pm}}$comparing individual SiMSSM points to an overall fit. The fit agrees well with the individual points, so we use the overall fit for each of these points. In each plot the solid line is the fit to that particular set of points and the dashed line is a fit to all points with $\tan \beta=2$. 


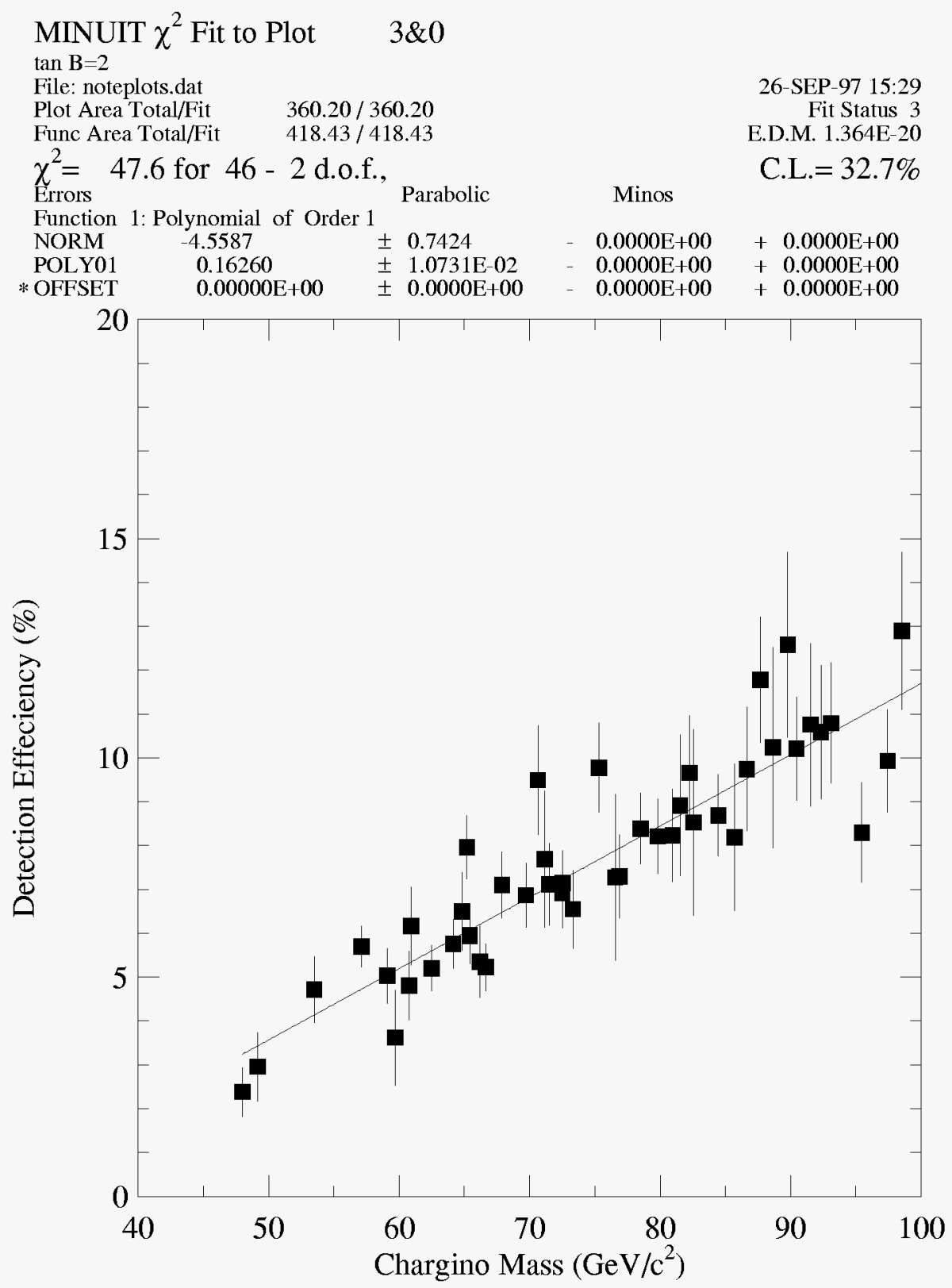

Figure 5.4: The detection efficiency as a function of $\mathrm{M}_{\tilde{\chi}_{1}^{ \pm}}$for a variety of points in SiMSSM space, constraining $\tan \beta=2$. The quality of the fit is better than when $\tan \beta$ is unconstrained. 
It is appropriate to wonder why we want a functional form for the detection efficiency. To determine the detection efficiency, we must generate and simulate at least 20,000 events. This can take some time. If the detection efficiency is independent of the model parameters, than we need only calculate the cross section and branching ratio for a particular SiMSSM point. This is a much less time consuming process. For points where $\tan \beta=2$, we use (see Figure 5.4)

$$
\epsilon_{\text {tot }}=-4.56+0.16 M_{\tilde{\chi}_{1}^{ \pm}} .
$$

For all other points we use the $\epsilon_{\text {tot }}$ determined from Monte Carlo for that particular point. If we determine $\epsilon_{\text {tot }}$ for each SiMSSM point, the total systematic uncertainty drops from $20 \%$ to $15 \%$. However, this only reduces $N_{e x p}$ from 3.2 to 3.1. The increased analysis speed is a great benefit, with only a small decrease in our limit.

\subsection{3 $\int \mathcal{L} d t:$ Integrated Luminosity}

This analysis is primarily concerned with the Run IB data. However, a similar analysis was done using the Run IA data [45]. That analysis used identical cuts (but did not apply the $\mathbb{E}_{T}$ cut) and had no events remaining. Thus, we can combine the $87.7 \mathrm{pb}^{-1}$ Run IB data with the $19.1 \mathrm{pb}^{-1}$ of Run IA data for a total of $107 \mathrm{pb}^{-1}$. We have no candidate events in the entire sample.

\subsection{Limits on the SiMSSM}

It is impractical for us to try to examine the entire SiMSSM parameter space, even after reducing to the six free parameters discussed in Chapter 2. We can further reduce the number of free parameters to four by noting that this analysis is insensitive to the top trilinear coupling $\left(A_{T}\right)$ and the mass of the pseudoscalar Higgs boson $\left(H_{A}\right)$. We set $A_{T}=\mu / \tan \beta^{5}$ and $M_{H_{A}}=500 \mathrm{GeV} / \mathrm{c}^{2}{ }^{6}{ }^{6}$ The remaining parameters are $\mu, \tan \beta, \mathrm{M}_{\tilde{q}}$ and $\mathrm{M}_{\tilde{g}}$. Since the $\tilde{\ell}$ mass is mainly a function of $\Delta\left(M_{\tilde{q}}, M_{\tilde{g}}\right)$ we replace $\mathrm{M}_{\tilde{q}}$ with $\mathrm{M}_{\tilde{q}} / \mathrm{M}_{\tilde{g}}$. However, we still need guidance to determine which regions of parameter space we should examine.

This analysis yields the strongest limits when the leptonic branching ratios of the $\tilde{\chi}_{1}^{ \pm}$and $\tilde{\chi}_{2}^{0}$ are maximized. This occurs for two reasons: small Higgsino content of the $\tilde{\chi}_{1}^{ \pm}$and $\tilde{\chi}_{2}^{0}$ (as Higgsino-like $\tilde{\chi}_{1}^{ \pm}$and $\tilde{\chi}_{2}^{0}$ prefer to decay to quarks), and small $\tilde{\ell}$ masses (as light, but still virtual, sleptons can enhance the leptonic branching ratio). A quick look at Figures 2.12 and 2.15 reveals gaugino-like $\tilde{\chi}_{1}^{ \pm}$and $\tilde{\chi}_{2}^{0}$ for $|\mu| \gtrsim 200 \mathrm{GeV} / \mathrm{c}^{2}$ or $1 \lesssim \tan \beta \lesssim 10$. The $\tilde{\chi}_{1}^{ \pm}$and $\tilde{\chi}_{2}^{0}$ masses increase

\footnotetext{
${ }^{5}$ We use $A_{T}=\mu / \tan \beta$ as it gives the smallest stop splitting. This is not relevant to this analysis (as we ignore all but the first generation of squarks) but is used for consistency with other CDF analyses.

${ }^{6} \mathrm{We}$ use $M_{H_{A}}=500 \mathrm{GeV} / \mathrm{c}^{2}$ to remove any possibility of decay to Higgs. Any value $>100 \mathrm{GeV} / \mathrm{c}^{2}$ would be acceptable.
} 
with $|\mu|$, resulting in small production cross sections. We thus only study $|\mu|<1000 \mathrm{GeV} / \mathrm{c}^{2}$. As discussed in Section 2.3, large $\tilde{q}$ masses reduce the $t$-channel production mechanism, thus increasing the overall $\tilde{\chi}_{1}^{ \pm} \tilde{\chi}_{2}^{0}$ production cross section. However, since the $\widetilde{\ell}$ masses are determined mainly by the difference in $\tilde{q}$ and $\tilde{g}$ masses (Equation 2.2), increasing the $\tilde{q}$ mass yields heavier $\tilde{\ell}$. That in turn reduces the branching ratio to leptons. The reduction in branching ratio is a larger effect than the increase of production cross section. We thus want to keep the $\tilde{q}$ to $\tilde{g}$ mass ratio less than 2 . Finally, if the squarks are too much lighter than the gluinos, the renormalization group equations (Equation 2.2) break down. These formulae are useful for $\mathrm{M}_{\tilde{q}} / \mathrm{M}_{\tilde{g}}>0.90$. When the squarks are lighter than the gluinos, the sleptons are lighter than the $\tilde{\chi}_{1}^{ \pm}$and $\tilde{\chi}_{2}^{0}$. This results in two-body decays of the $\tilde{\chi}_{1}^{ \pm}$and $\tilde{\chi}_{2}^{0}$. However, for $0.90<\mathrm{M}_{\tilde{q}} / \mathrm{M}_{\tilde{g}} \lesssim 0.95$ the lightest slepton is a sneutrino and invisible decays of the $\tilde{\chi}_{1}^{ \pm}$and $\tilde{\chi}_{2}^{0}$ dominate. $\mathrm{M}_{\tilde{\chi}_{1}^{ \pm}}$and $\mathrm{M}_{\tilde{\chi}_{2}^{0}}$ are mainly functions of $\mathrm{M}_{\tilde{g}}$ so we set $\mathrm{M}_{\tilde{g}}$ to values that yield $45 \mathrm{GeV} / \mathrm{c}^{2} \lesssim \mathrm{M}_{\tilde{\chi}_{1}^{ \pm}} \lesssim 100 \mathrm{GeV} / \mathrm{c}^{2}$.

To summarize, we search the SiMSSM parameter space in the regions

- $-200 \mathrm{GeV} / \mathrm{c}^{2} \leq \mu \leq-1000 \mathrm{GeV} / \mathrm{c}^{2}$,

- $1.1 \leq \tan \beta \leq 8$,

- $0.95 \leq \mathrm{M}_{\tilde{q}} / \mathrm{M}_{\tilde{g}} \leq 2$, and

- $150 \mathrm{GeV} / \mathrm{c}^{2} \leq \mathrm{M}_{\tilde{g}} \leq 340 \mathrm{GeV} / \mathrm{c}^{2}$.

Our limits for the SiMSSM parameter space outside these regions are weaker and thus less interesting. For all points we examine we find $\mathrm{M}_{\tilde{\chi}_{1}^{ \pm}} \approx \mathrm{M}_{\tilde{\chi}_{2}^{0}} \approx 2 \mathrm{M}_{\tilde{\chi}_{1}}$.

In Figures 5.5-5.16 we present the 95\% Confidence Level (C.L.) upper limit curve and compare it to the predicted cross section times branching ratio for a variety of points in SiMSSM space. The $95 \%$ C.L. upper limit curve is a line indicating the minimum cross section times branching ratio we can detect as a function of $\tilde{\chi}_{1}^{ \pm}$mass $^{7}$. Above the line enough events would be produced so we could detect them; below it we cannot. This line is the right hand side of Equation 5.1. The cross section times branching ratio lines are calculated using ISAJET 7.20 and are the production cross section for $p \bar{p} \rightarrow \tilde{\chi}_{1}^{ \pm} \tilde{\chi}_{2}^{0}$ times the branching ratio $\tilde{\chi}_{1}^{ \pm} \tilde{\chi}_{2}^{0} \rightarrow 3 \ell+X$, where $\ell$ is either $e$ or $\mu$. It is important to realize the branching ratio is the sum of all four possible $e$ or $\mu$ combinations (eee, $e e \mu, e \mu \mu, \mu \mu \mu$ ). Figure 5.5 shows the 95\% C.L. upper limit curve (solid line) in comparison to cross section times branch ratio curves (dashed lines) for $\mathrm{M}_{\tilde{q}} / \mathrm{M}_{\tilde{g}}=1,1.2,1.5$ and 2.0. The mass lower limit and $\sigma_{\tilde{\chi}_{1}^{ \pm} \tilde{\chi}_{2}^{0}} \cdot \operatorname{BR}\left(\tilde{\chi}_{1}^{ \pm} \tilde{\chi}_{2}^{0} \rightarrow 3 \ell+X\right)$ upper limit can be read off the graph where the dashed and solid lines intersect. Clearly, as the squarks become heavier than the gluinos, the limit degrades.

\footnotetext{
${ }^{7}$ The $95 \%$ indicates we would detect events in 95 experiments out of 100 performed; it does not mean we are $95 \%$ sure about our result.
} 
For the case where $\mathrm{M}_{\tilde{q}} / \mathrm{M}_{\tilde{g}}=0.95$ we have two-body decays of the $\tilde{\chi}_{1}^{ \pm}$and $\tilde{\chi}_{2}^{0}$, so the acceptances are somewhat different. Figure 5.6 shows we set no limit for $\mathrm{M}_{\tilde{q}} / \mathrm{M}_{\tilde{g}}=0.95$. Figure 5.7 is the lower limit on $\mathrm{M}_{\tilde{\chi}_{1}^{ \pm}}$as a function of $\mathrm{M}_{\tilde{q}} / \mathrm{M}_{\tilde{g}}$. For all of these points we hold both $\tan \beta$ and $\mu$ constant and set them to 2 and $-400 \mathrm{GeV} / \mathrm{c}^{2}$, respectively. We also compare our $\sigma_{\tilde{\chi}_{1}^{ \pm} \tilde{\chi}_{2}^{0}} \cdot \operatorname{BR}\left(\tilde{\chi}_{1}^{ \pm} \tilde{\chi}_{2}^{0} \rightarrow 3 \ell+X\right)$ limit to that of D0 $[46]^{8}$. Their limit is weaker than ours for two reasons: they have somewhat poorer muon detection abilities, and use a higher $P_{T}$ threshold for all of their leptons. It is very important to realize that every experiment makes slightly different assumptions about models and so limits cannot always be easily compared. D0 only examines SUGRA models (see below for that comparison).

Figure 5.8 compares the $95 \%$ C.L. line to a variety of $\mu$ values: $-200,-400$ and $-800 \mathrm{GeV} / \mathrm{c}^{2}$. For this plot we set $\tan \beta=2$ and $\mathrm{M}_{\tilde{q}} / \mathrm{M}_{\tilde{g}}=2$. Recall that $|\mu|$ is the unmixed Higgsino mass, so as $\mu$ increases the Higgsino content of the $\tilde{\chi}_{1}^{ \pm}$and $\tilde{\chi}_{2}^{0}$ will decrease. However, as $|\mu|$ gets large, the masses of the $\tilde{\chi}_{1}^{ \pm}$and $\tilde{\chi}_{2}^{0}$ tends to increase. This decreases the production cross section, reducing our limit. This can be seen in Figure 5.8. Figure 5.9 is the lower limit on $\mathrm{M}_{\tilde{\chi}_{1}^{ \pm}}$as a function of $\mu$. For comparison, we indicate the ALEPH limit [47]. It is important to note that the ALEPH limit includes searches for hadronic final states of the $\tilde{\chi}_{1}^{ \pm}$and $\tilde{\chi}_{2}^{0}$, as well as the leptonic signature we use. Also, the LEP experiments (since LEP in an $e^{+} e^{-}$machine) are more sensitive to low values of $|\mu|$ while Tevatron experiments are more sensitive to high values of $|\mu|$. As with the D0 limit, ALEPH makes somewhat different model assumptions. They examine only SUGRA, which (see below) favors low $|\mu|$ values. However, their kinematic limit is quite model independent and can be compared with our limit.

Having varied both $\mathrm{M}_{\tilde{q}} / \mathrm{M}_{\tilde{g}}$ and $\mu$, we now vary $\tan \beta$. Since the acceptance changes with $\tan \beta$, each $95 \%$ C.L. curve is on a separate plot. We show plots for $\tan \beta=1.1,1.5,2,4$ and 8 . For these points we set $\mathrm{M}_{\tilde{q}} / \mathrm{M}_{\tilde{g}}$ and $\mu$ to 1 and $-400 \mathrm{GeV} / \mathrm{c}^{2}$, respectively. Figures 5.10-5.14 show the individual $\sigma \cdot B R$ plots for each $\tan \beta$ point and Figure 5.15 summarizes the lower limit on $\mathrm{M}_{\tilde{\chi}_{1}^{ \pm}}$as a function of $\tan \beta$. As expected, the limit decreases as $\tan \beta$ increases.

We have also examined the $\tilde{\chi}_{1}^{ \pm}$mass limit for high $\tilde{q}$ mass. This region is interesting since, as mentioned above, the $t$-channel virtual $\tilde{q}$ exchange interferes destructively with the $s$-channel $W^{ \pm}$exchange. Regions of high $\tilde{q}$ mass effectively turn off $t$-channel production. Figure 5.16 shows the limit on $\mathrm{M}_{\tilde{\chi}_{1}^{ \pm}}$for $\mathrm{M}_{\tilde{q}}=500 \mathrm{GeV} / \mathrm{c}^{2}$. For this plot we fix $\mathrm{M}_{\tilde{q}}$ and allow $\mathrm{M}_{\tilde{g}}$ to vary freely. Again, high $\tilde{q}$ masses lead to high $\tilde{\ell}$ masses, which lead to somewhat degraded limits.

Tables 5.2-5.6 list the input $\left(\mathrm{M}_{\tilde{q}}, \mathrm{M}_{\tilde{g}}, \tan \beta\right.$ and $\left.\mu\right)$ and the relevant output parameters $\left(\mathrm{M}_{\tilde{\chi}_{1}^{0}}\right.$, $\mathrm{M}_{\tilde{\chi}_{2}^{0}}, \mathrm{M}_{\tilde{\chi}_{1}^{ \pm}}, \mathrm{M}_{\widetilde{\ell}_{L}}, \mathrm{M}_{\widetilde{\nu}_{L}}, \mathrm{M}_{\widetilde{\ell}_{R}}$ and the branching ratios to leptons) for each point examined in SiMSSM

${ }^{8} \mathrm{D} 0$ plots their limit for an average branching ratio to leptons; we use a sum of branching ratios to $e$ or $\mu$. Thus, their result is scaled by a factor of 4 for comparison with ours. 


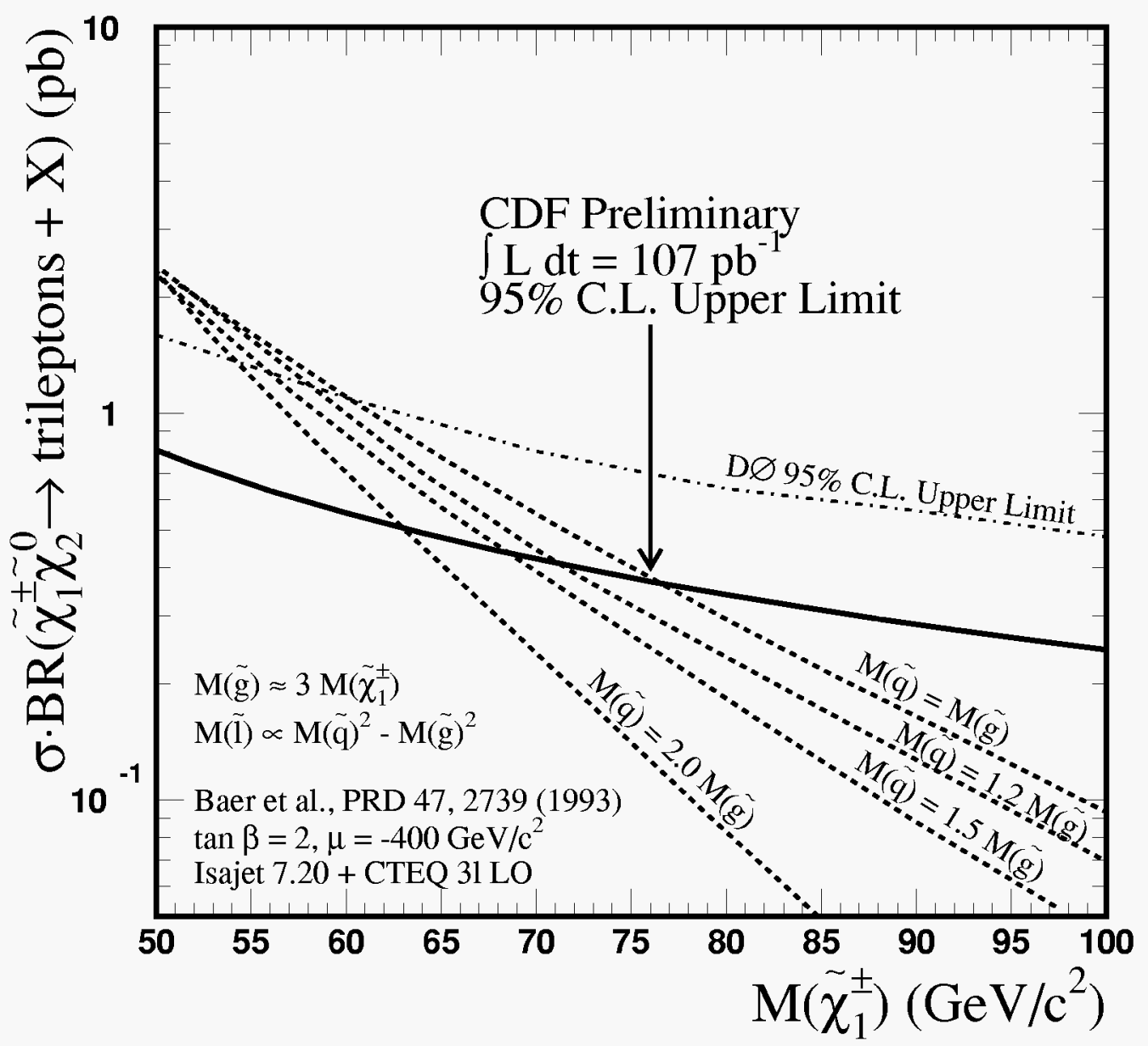

Figure 5.5: Production cross section times branching ratio to leptons $(e$ or $\mu)$ for $\tilde{\chi}_{1}^{ \pm} \tilde{\chi}_{2}^{0}$ production as a function of $\tilde{\chi}_{1}^{ \pm}$mass for $\tan \beta=2$, and $\mu=-400 \mathrm{GeV} / \mathrm{c}^{2}$. The dashed lines are the theoretical prediction and the solid line is the $95 \%$ Confidence Level limit (using the fit to all $\tan \beta=2$ points). We use ISAJET 7.20 with the CTEQ 3L structure functions to generate the Monte Carlo. As expected, the limit worsens as the squarks become much heavier than the gluinos. For comparison, we indicate the D0 95\% C.L. limit [46]. 


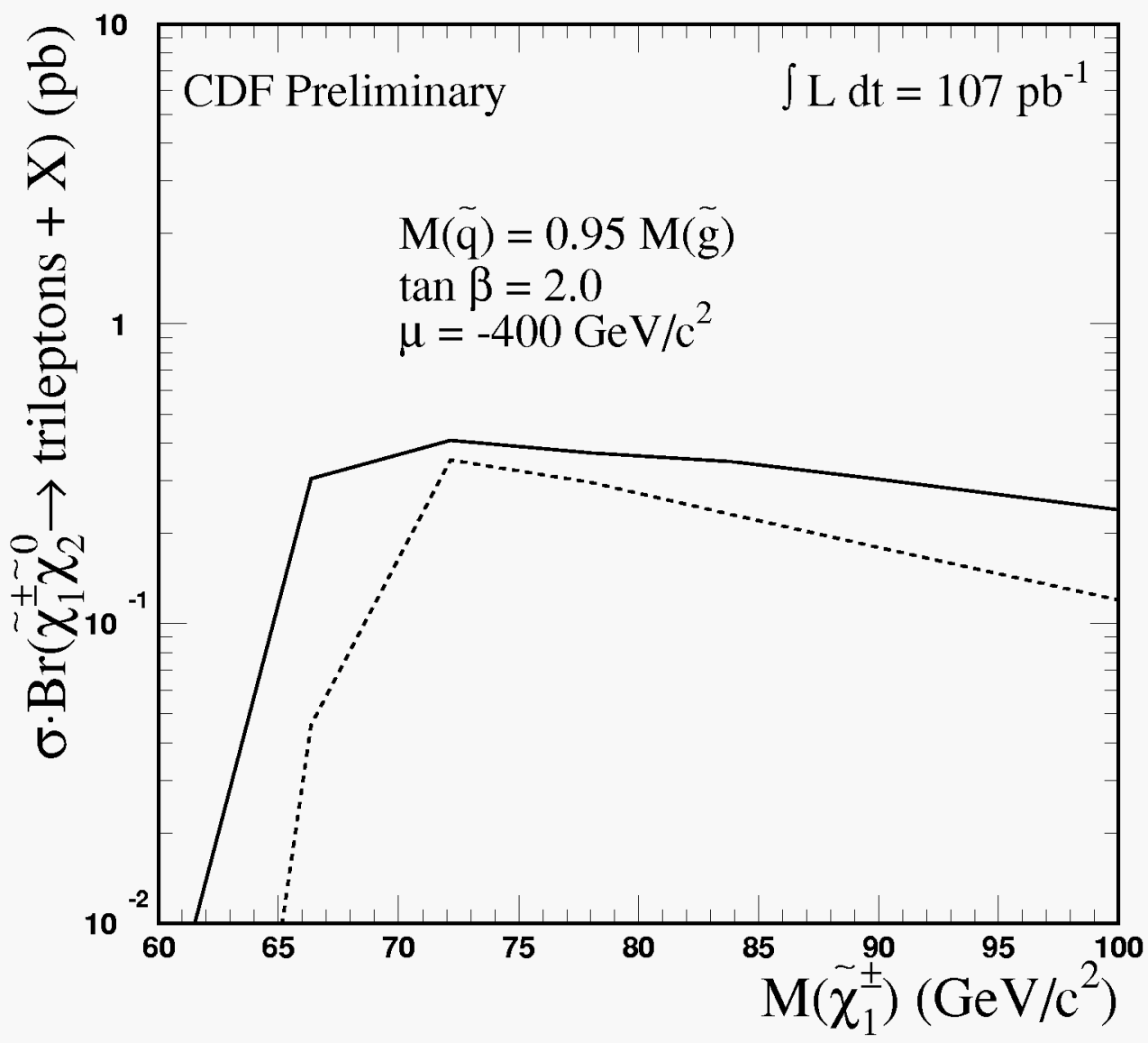

Figure 5.6: Production cross section times branching ratio to leptons $(e$ or $\mu)$ for $\tilde{\chi}_{1}^{ \pm} \tilde{\chi}_{2}^{0}$ production as a function of $\tilde{\chi}_{1}^{ \pm}$mass for $\tan \beta=2, \mathrm{M}_{\tilde{q}} / \mathrm{M}_{\tilde{q}}=0.95$ and $\mu=-400 \mathrm{GeV} / \mathrm{c}^{2}$. The dashed line is the theoretical prediction and the solid line is the 95\% Confidence Level limit. We use ISAJET 7.20 with the CTEQ 3L structure functions to generate the Monte Carlo. We do not set a mass limit at this point; at low chargino masses the sneutrino is lighter than the sleptons so invisible decays dominate. 


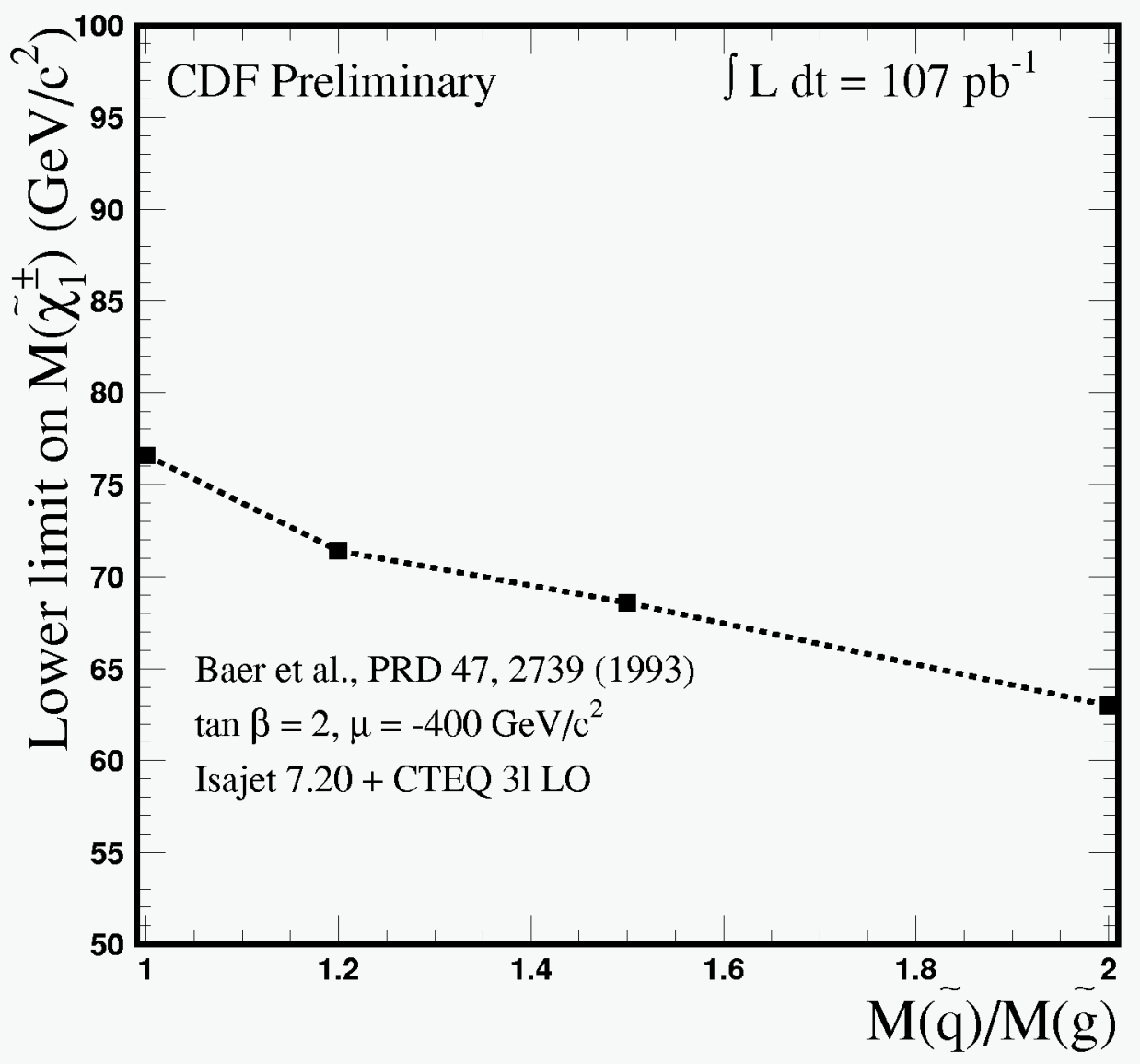

Figure 5.7: Limit on $\mathrm{M}_{\tilde{\chi}_{1}^{ \pm}}$as a function of $\mathrm{M}_{\tilde{q}} / \mathrm{M}_{\tilde{g}}$. The limit decreases as the $\tilde{q} \mathrm{~s}$ become much more massive than the $\tilde{g}$ s since the $\tilde{\ell}$ masses depend on the difference between them. Heavier $\tilde{\ell}_{\mathrm{s}}$ lead to fewer decays via virtual $\widetilde{\ell}_{s}$ and thus a smaller branching ratio to leptons. 


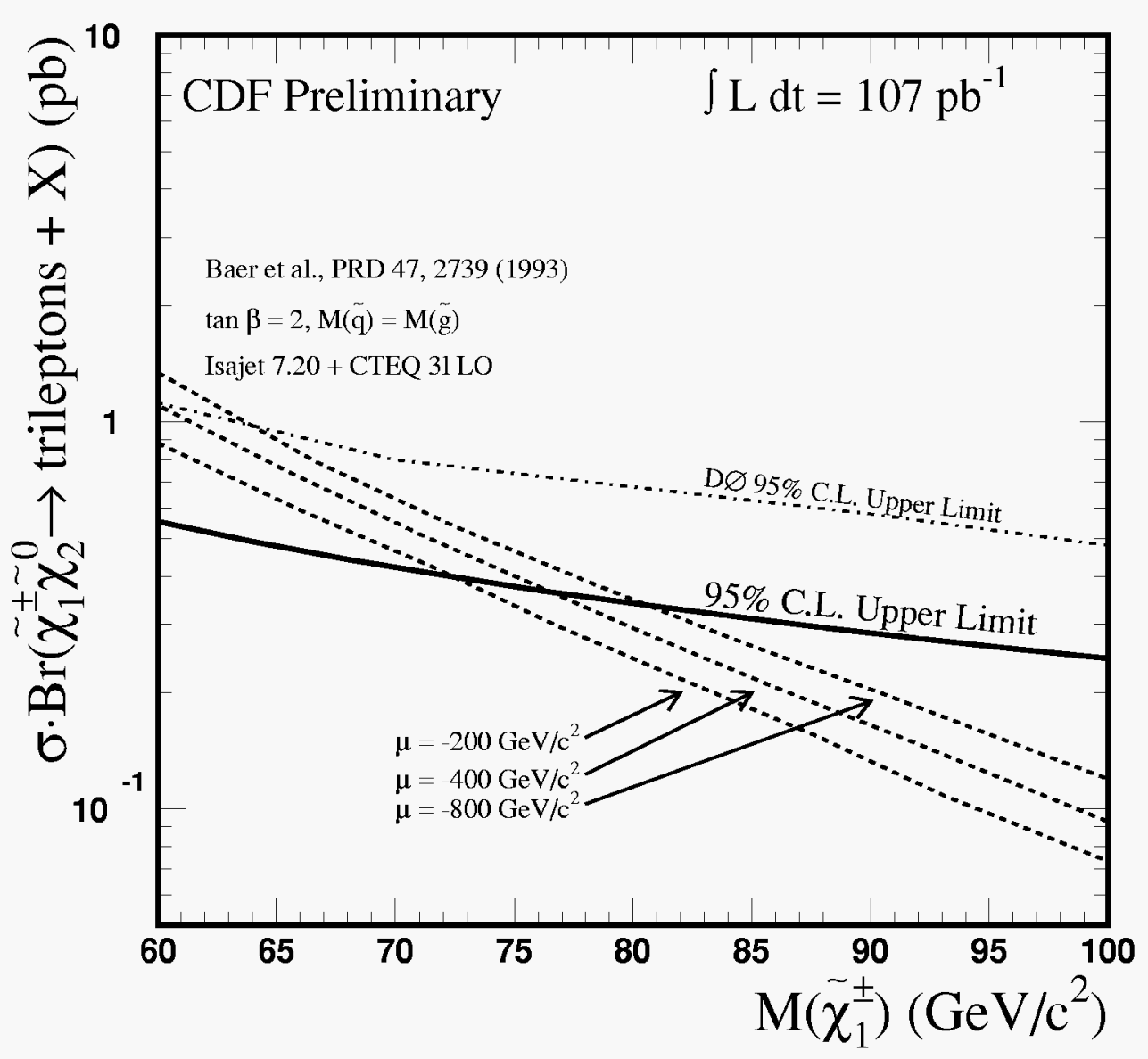

Figure 5.8: Production cross section times branching ratio to leptons $\left(e\right.$ or $\mu$ ) for $\tilde{\chi}_{1}^{ \pm} \tilde{\chi}_{2}^{0}$ production as a function of $\tilde{\chi}_{1}^{ \pm}$mass for $\tan \beta=2$, and $\mathrm{M}_{\tilde{q}}=\mathrm{M}_{\tilde{g}}$. The dashed lines are the theoretical prediction and the solid line is the $95 \%$ Confidence Level limit (using the fit to all $\tan \beta=2$ points). We use ISAJET 7.20 with the CTEQ 3L structure functions to generate the Monte Carlo. For comparison, we indicate the D0 95\% C.L. limit [46]. 


\section{Search for SUSY using $\mathbf{p} \overline{\mathbf{p}} \rightarrow \mathbf{e e e}, \mathbf{e e} \mu, \mathbf{e} \mu \mu, \mu \mu \mu$}

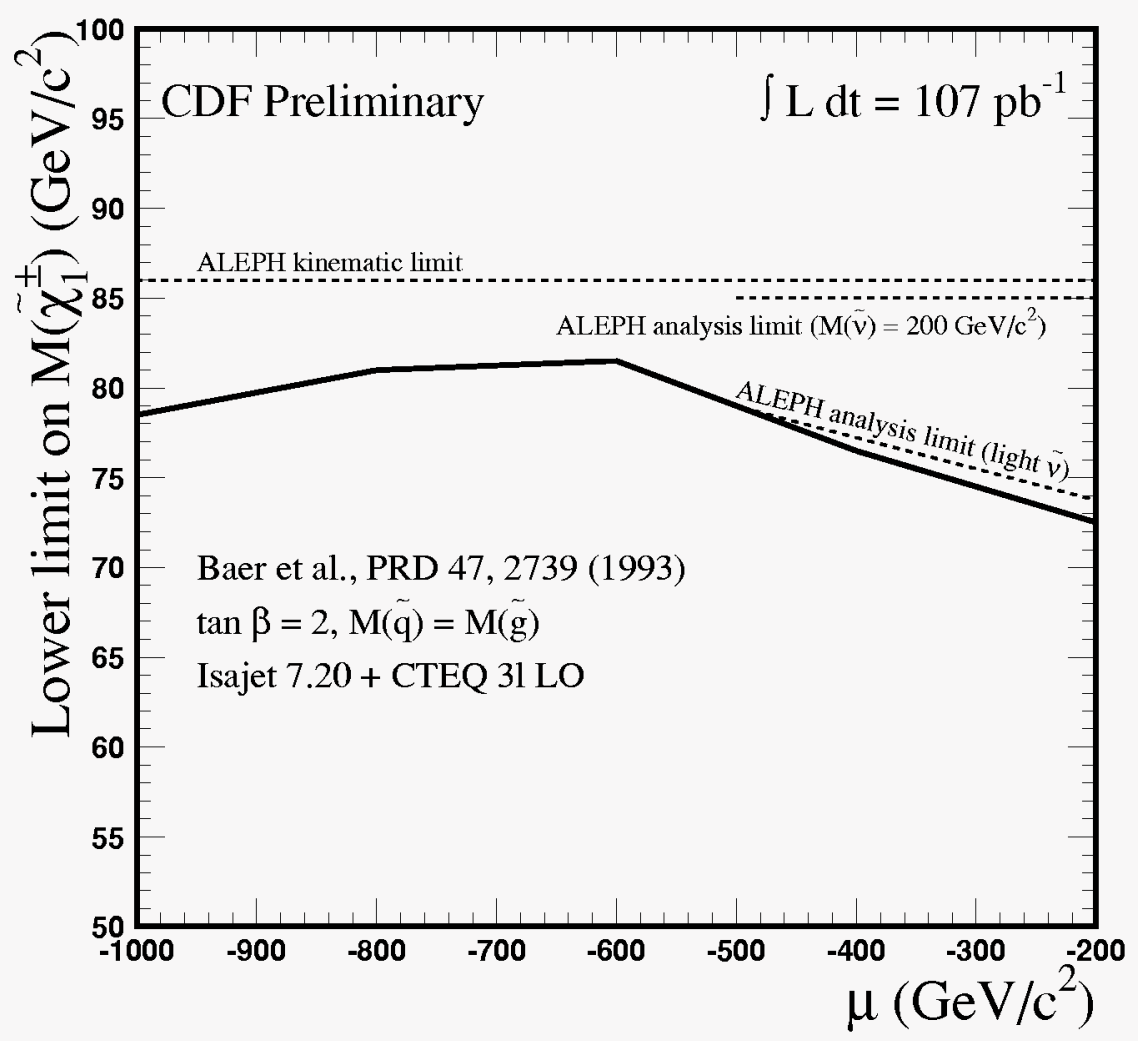

Figure 5.9: The solid curve is the limit on $\mathrm{M}_{\tilde{\chi}_{1}^{ \pm}}$as a function of $\mu$. The maximum limit is 81.5 $\mathrm{GeV} / \mathrm{c}^{2}$, for $\mu=-600 \mathrm{GeV} / \mathrm{c}^{2}$. For comparison, we show the ALEPH limit (dashed lines) [47]. The kinematic limit is half the LEP-II center-of-mass energy. It is important to realize that ALEPH searches for several final states, not only the trilepton state searched for in this analysis. Just as the $s$ - and $t$-channels interfere at the Tevatron, so do they at LEP (where the production mechanism is via virtual sneutrino exchange). Thus, higher $\widetilde{\nu}$ masses result in higher production cross sections, but since ALEPH searches for all possible final states, they do not have the degradation in branching ratio we do. For the best comparison, we include the ALEPH limit for light $\widetilde{\nu}$. Also, ALEPH only examines SUGRA, which tends to keep $|\mu|<500 \mathrm{GeV} / \mathrm{c}^{2}$. 


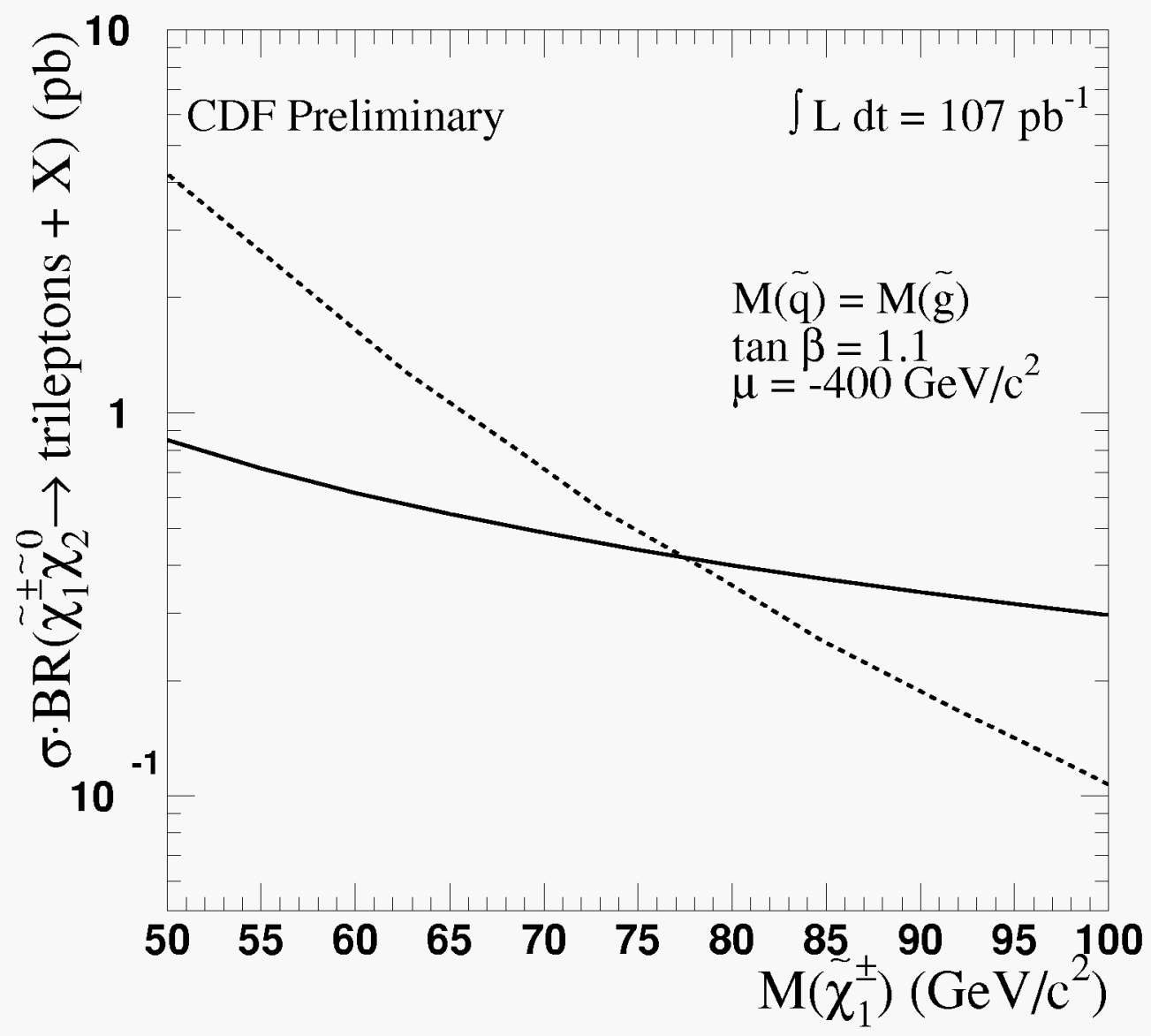

Figure 5.10: Production cross section times branching ratio to leptons $(e$ or $\mu)$ for $\tilde{\chi}_{1}^{ \pm} \tilde{\chi}_{2}^{0}$ production as a function of $\tilde{\chi}_{1}^{ \pm}$mass for $\tan \beta=1.1, \mathrm{M}(\tilde{q})=\mathrm{M}(\tilde{g})$ and $\mu=-400 \mathrm{GeV} / \mathrm{c}^{2}$. The dashed line is the theoretical prediction and the solid line is the $95 \%$ Confidence Level limit. We use ISAJET 7.20 with the CTEQ 3L structure functions to generate the Monte Carlo. 


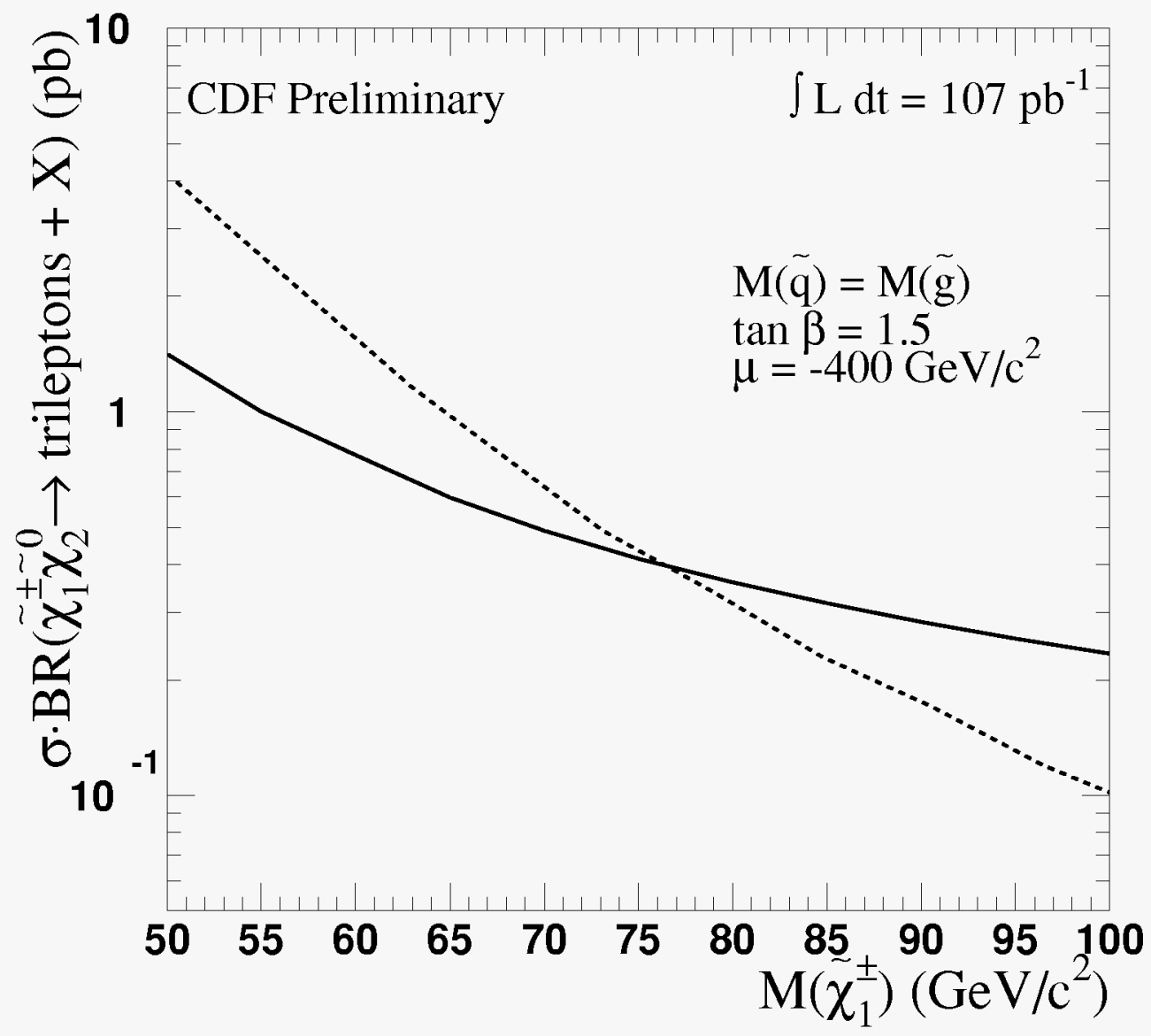

Figure 5.11: Production cross section times branching ratio to leptons $(e$ or $\mu)$ for $\tilde{\chi}_{1}^{ \pm} \tilde{\chi}_{2}^{0}$ production as a function of $\tilde{\chi}_{1}^{ \pm}$mass for $\tan \beta=1.5, \mathrm{M}(\tilde{q})=\mathrm{M}(\tilde{g})$ and $\mu=-400 \mathrm{GeV} / \mathrm{c}^{2}$. The dashed line is the theoretical prediction and the solid line is the $95 \%$ Confidence Level limit. We use ISAJET 7.20 with the CTEQ 3L structure functions to generate the Monte Carlo. 


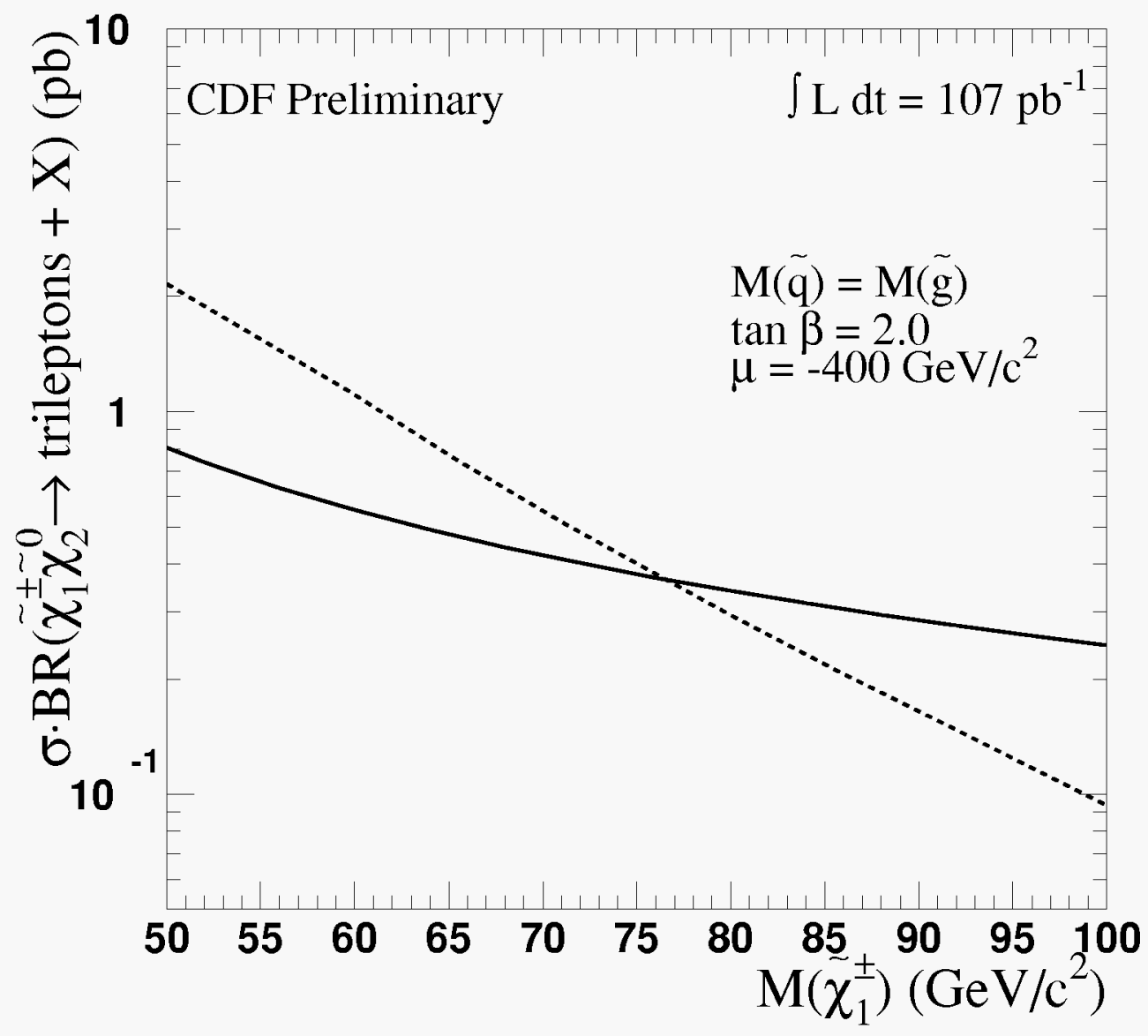

Figure 5.12: Production cross section times branching ratio to leptons $(e$ or $\mu)$ for $\tilde{\chi}_{1}^{ \pm} \tilde{\chi}_{2}^{0}$ production as a function of $\tilde{\chi}_{1}^{ \pm}$mass for $\tan \beta=2, \mathrm{M}(\tilde{q})=\mathrm{M}(\tilde{g})$ and $\mu=-400 \mathrm{GeV} / \mathrm{c}^{2}$. The dashed line is the theoretical prediction and the solid line is the $95 \%$ Confidence Level limit. We use ISAJET 7.20 with the CTEQ 3L structure functions to generate the Monte Carlo. 


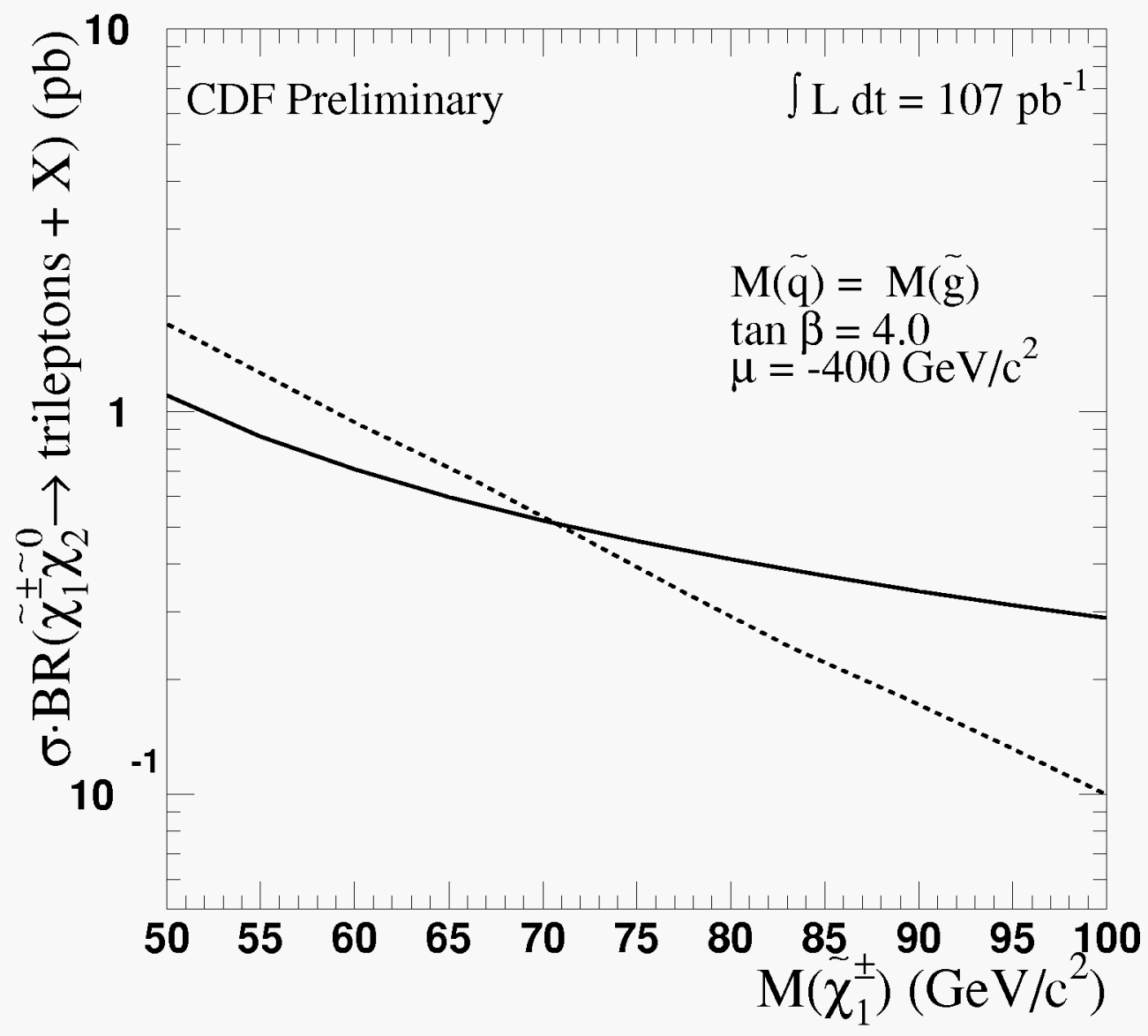

Figure 5.13: Production cross section times branching ratio to leptons $(e$ or $\mu)$ for $\tilde{\chi}_{1}^{ \pm} \tilde{\chi}_{2}^{0}$ production as a function of $\tilde{\chi}_{1}^{ \pm}$mass for $\tan \beta=4, \mathrm{M}(\tilde{q})=\mathrm{M}(\tilde{g})$ and $\mu=-400 \mathrm{GeV} / \mathrm{c}^{2}$. The dashed line is the theoretical prediction and the solid line is the $95 \%$ Confidence Level limit. We use ISAJET 7.20 with the CTEQ 3L structure functions to generate the Monte Carlo. 


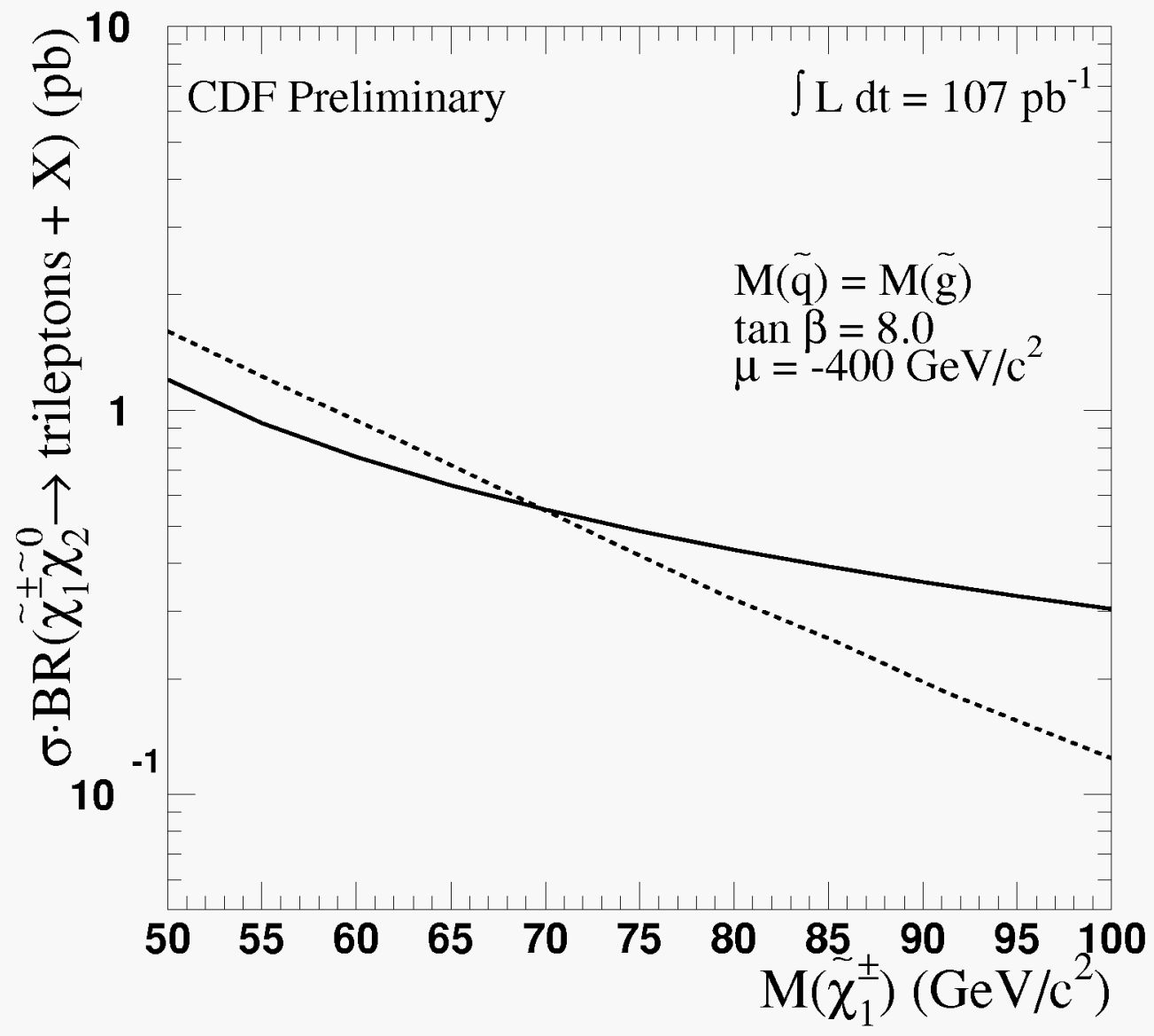

Figure 5.14: Production cross section times branching ratio to leptons $(e$ or $\mu)$ for $\tilde{\chi}_{1}^{ \pm} \tilde{\chi}_{2}^{0}$ production as a function of $\tilde{\chi}_{1}^{ \pm}$mass for $\tan \beta=8, \mathrm{M}(\tilde{q})=\mathrm{M}(\tilde{g})$ and $\mu=-400 \mathrm{GeV} / \mathrm{c}^{2}$. The dashed line is the theoretical prediction and the solid line is the $95 \%$ Confidence Level limit. We use ISAJET 7.20 with the CTEQ 3L structure functions to generate the Monte Carlo. 


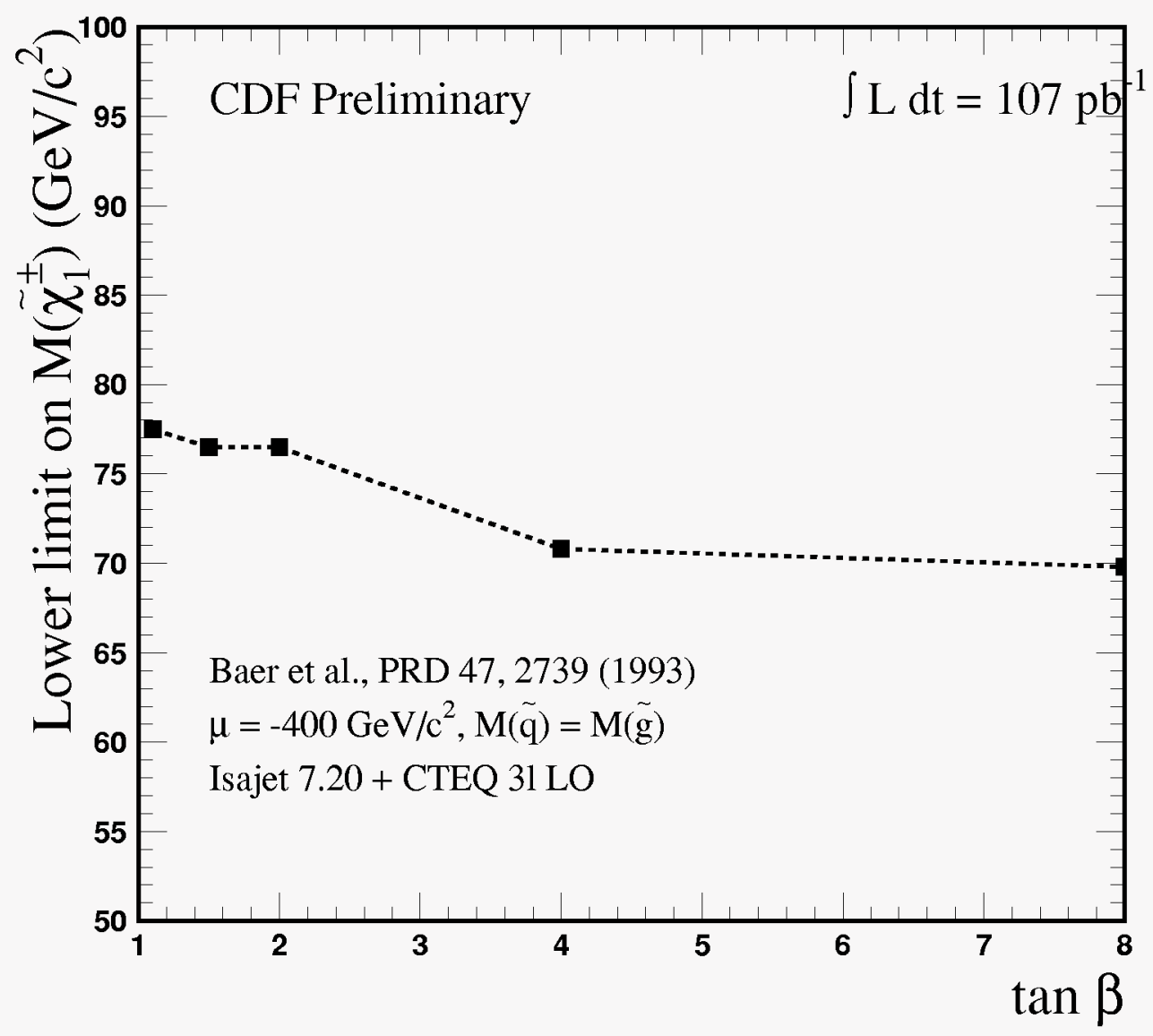

Figure 5.15: Lower limit on $\mathrm{M}\left(\tilde{\chi}_{1}^{ \pm}\right)$as a function of $\tan \beta$ for, $\mathrm{M}(\tilde{q})=\mathrm{M}(\tilde{g})$ and $\mu=-400 \mathrm{GeV} / \mathrm{c}^{2}$. As expected, the limit decreases as $\tan \beta$ increases. 


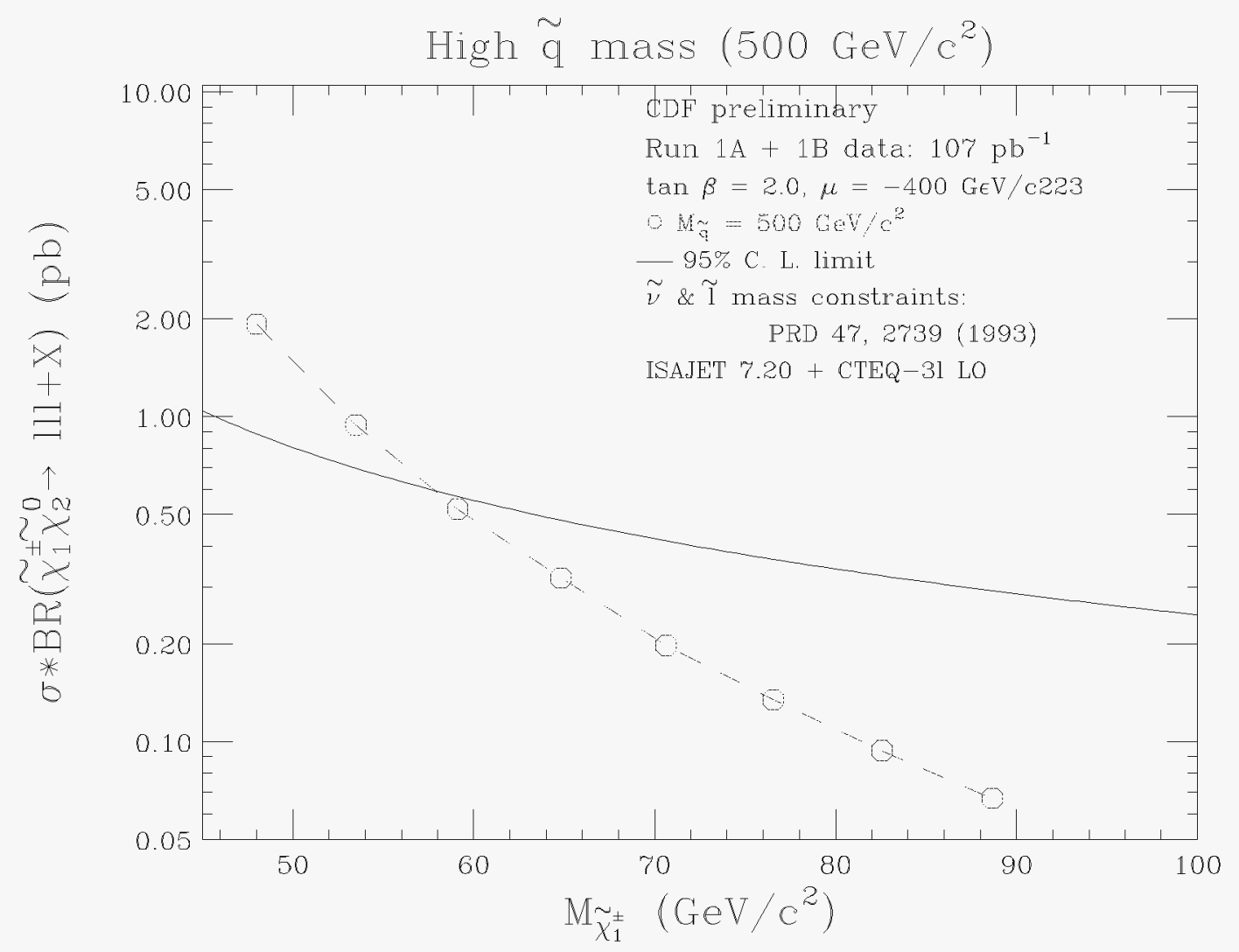

Figure 5.16: Production cross section times branching ratio to leptons ( $e$ or $\mu$ ) for $\tilde{\chi}_{1}^{ \pm} \tilde{\chi}_{2}^{0}$ production as a function of $\tilde{\chi}_{1}^{ \pm}$mass for $\tan \beta=2$ and $\mu=-400 \mathrm{GeV} / \mathrm{c}^{2}$ and $\mathrm{M}_{\tilde{q}}=500 \mathrm{GeV} / \mathrm{c}^{2}$. The dashed line is the theoretical prediction and the solid line is the $95 \%$ Confidence Level limit (using the fit to all $\tan \beta=2$ points). We use ISAJET 7.20 with the CTEQ 3L structure functions to generate the Monte Carlo. 
Table 5.2: Input and output parameters for the SiMSSM points scanned. All masses are in $\mathrm{GeV} / \mathrm{c}^{2}$ and all $\sigma$ are in picobarns.

\begin{tabular}{|c|c|c|c|c|c|c|c|c|c|c|}
\hline & \multicolumn{2}{|c|}{ Input } & \multicolumn{8}{|c|}{ Output } \\
\hline Set & $\begin{array}{c}\tilde{q} \\
(\mathrm{GeV})\end{array}$ & $\begin{array}{c}\tilde{g} \\
\left.V / \mathrm{c}^{2}\right)\end{array}$ & $\begin{array}{cccc}\tilde{\chi}_{1}^{0} & \tilde{\chi}_{2}^{0} & \tilde{\chi}_{1}^{ \pm} & \widetilde{\ell}_{L} \\
& & \left(\mathrm{GeV} / \mathrm{c}^{2}\right)\end{array}$ & $\widetilde{\nu}$ & & $\begin{array}{r}\mathrm{BR} \rightarrow \\
\tilde{\chi}_{2}^{0} \\
\end{array}$ & $\begin{array}{r}\mathrm{BR} \rightarrow \\
\tilde{\chi}_{1}^{ \pm}\end{array}$ & $\begin{array}{c}\mathrm{BR} \rightarrow 3 \ell \\
\text { Total }\end{array}$ & $\sigma$ & $\sigma \cdot \mathrm{BR}$ \\
\hline \multicolumn{11}{|c|}{$\mathrm{M}_{\tilde{q}} \simeq \mathrm{M}_{\tilde{g}}, \mu=-400 \mathrm{GeV} / \mathrm{c}^{2}, \tan \beta=2.0$} \\
\hline 14.1 & 180 & 179 & .759 .7101 .8 & 80.7 & 92.6 & 0.321 & 0.461 & 0.148 & 7.66 & 1.13 \\
\hline 11 & 200 & 199 & 9.766 .365 .4111 .5 & 92.7 & 101.3 & 0.348 & 0.433 & 51 & .98 & 0.75 \\
\hline 12 & 199 & 200 & $3.966 .7 \quad 65.8 \quad 108.4$ & 88.9 & 97.7 & 0.331 & 0.455 & 1 & .80 & 0.72 \\
\hline 14.2 & 220 & 219 & $2.771 .971 .1 \quad 121.4$ & 104.4 & 110.2 & 0.364 & 0.409 & & .42 & 0.5 \\
\hline 13 & 250 & 249 & 6.3 & 121.4 & 123.6 & 0.377 & 0.377 & & 09 & 0.3 \\
\hline 14 & 249 & 250 & 0.2 & 117.8 & 120.0 & 0.370 & 0.393 & & 03 & 0.3 \\
\hline 14.3 & 270 & 269 & 5.7 & 132.6 & 132.7 & 0.381 & 0.359 & & .53 & 0.21 \\
\hline 14.4 & 290 & 289 & 6.4 & 143.6 & 141.8 & 0.384 & 0.342 & & .15 & 0.15 \\
\hline 14.5 & 310 & 309 & \begin{tabular}{|llll}
46.497 .9 & 97.4 & 166.6
\end{tabular} & 154.6 & 150.9 & 0.384 & 0.326 & 0.125 & 0.88 & 0.11 \\
\hline \multicolumn{11}{|c|}{$\operatorname{High} \mathrm{M}_{\tilde{q}}\left(\mathrm{M}_{\tilde{q}}=500 \mathrm{GeV} / \mathrm{c}^{2}\right), \mu=-400 \mathrm{GeV} / \mathrm{c}^{2}, \tan \beta=2.0$} \\
\hline 15 & 500 & 160 & 20.5 & 170.1 & 480.8 & 0.212 & 0.222 & 0.047 & 1.08 & 1.93 \\
\hline 16 & 500 & 180 & 11.2 & J.1 & 475.3 & 0.198 & 222 & & 1.50 & 0.94 \\
\hline 17 & 500 & 200 & 1.3 & 401.2 & 469.0 & 0.187 & 0.222 & & 12.58 & 0.52 \\
\hline 18 & 500 & 220 & 34.8 & 400.0 & 462.0 & 0.178 & 0.222 & & 8.11 & 0.32 \\
\hline 19 & 500 & 240 & .6457 .5 & 453.3 & 454.1 & 0.171 & 0.221 & & 5.23 & 0.20 \\
\hline 19.1 & 500 & 260 & 3.6449 .4 & 440 & 445.4 & 0.166 & 0.221 & & 2.61 & 0.09 \\
\hline 19.2 & 500 & 280 & $\begin{array}{llll}38.7 & 83.2 & 82.6 & 440.6\end{array}$ & 436.2 & 435.9 & 0.163 & 0.221 & & 3.67 & 0.13 \\
\hline 19.3 & 500 & 300 & \begin{tabular}{|llll}
41.8 & 89.2 & 88.6 & 430.9 \\
\end{tabular} & 426.4 & 425.4 & 0.162 & 0.221 & 0.036 & 1.87 & 0.07 \\
\hline \multicolumn{11}{|c|}{$\mathrm{M}_{\tilde{q}}=1.2 \mathrm{M}_{\tilde{g}}, \mu=-400 \mathrm{GeV} / \mathrm{c}^{2}, \tan \beta=2.0$} \\
\hline 34.1 & 180 & 150 & $.751 .5 \quad 50.3 \quad 131.6$ & 116.1 & 126.5 & 0.421 & 0.292 & 0.122 & 23.96 & 2.94 \\
\hline 30 & 240 & 200 & $064.1 \quad 172.5$ & 161.0 & 166.0 & 0.418 & 0.261 & 09 & 6.40 & 0.70 \\
\hline 31 & 264 & 220 & $.070 .5 \quad 69.7 \quad 189.0$ & 178.5 & 181.9 & 0.411 & 0.253 & 04 & 4.37 & 0.45 \\
\hline 32 & 276 & 230 & $\begin{array}{llll}5 & 73.3 & 72.5 & 197.3\end{array}$ & 187.3 & 189.9 & 0.407 & 0.249 & 02 & 3.68 & 0.37 \\
\hline 33 & 288 & 240 & 1.976 .075 .3205 .5 & 196.0 & 197.9 & 0.403 & 0.246 & 99 & 3.16 & 0.31 \\
\hline 34 & 312 & 260 & |37.9 81.681 .0222 .1 & 213.3 & 213.9 & 0.392 & 0.241 & 0.094 & 2.34 & 0.22 \\
\hline 34.2 & 336 & 280 & \begin{tabular}{|llll}
40.8 & 87.2 & 86.6 & 238.8
\end{tabular} & 230.6 & 230.0 & 0.379 & 0.237 & 0.090 & 1.85 & 0.17 \\
\hline 34.3 & 360 & 300 & $43.792 .8 \quad 92.3 \quad 255.4$ & 247.8 & 246.1 & 0.363 & 0.233 & 0.084 & 1.31 & 0.11 \\
\hline
\end{tabular}

space.

\subsection{Limits on other models}

The limits we set above are rather general. We can also examine somewhat more specific models and set limits on them. We examine two models: a $\mathrm{SU}(5) \times \mathrm{U}(1)$ Supergravity model and a somewhat more general supergravity model known simply as SUGRA.

\subsection{1 $\mathrm{SU}(5) \times \mathrm{U}(1)$ Supergravity model}

The string-derived $\mathrm{SU}(5) \times \mathrm{U}(1)$ one parameter supergravity model [48] predicts 
Table 5.3: Input and output parameters for the SiMSSM points scanned. All masses are in $\mathrm{GeV} / \mathrm{c}^{2}$ and all $\sigma$ are in picobarns.

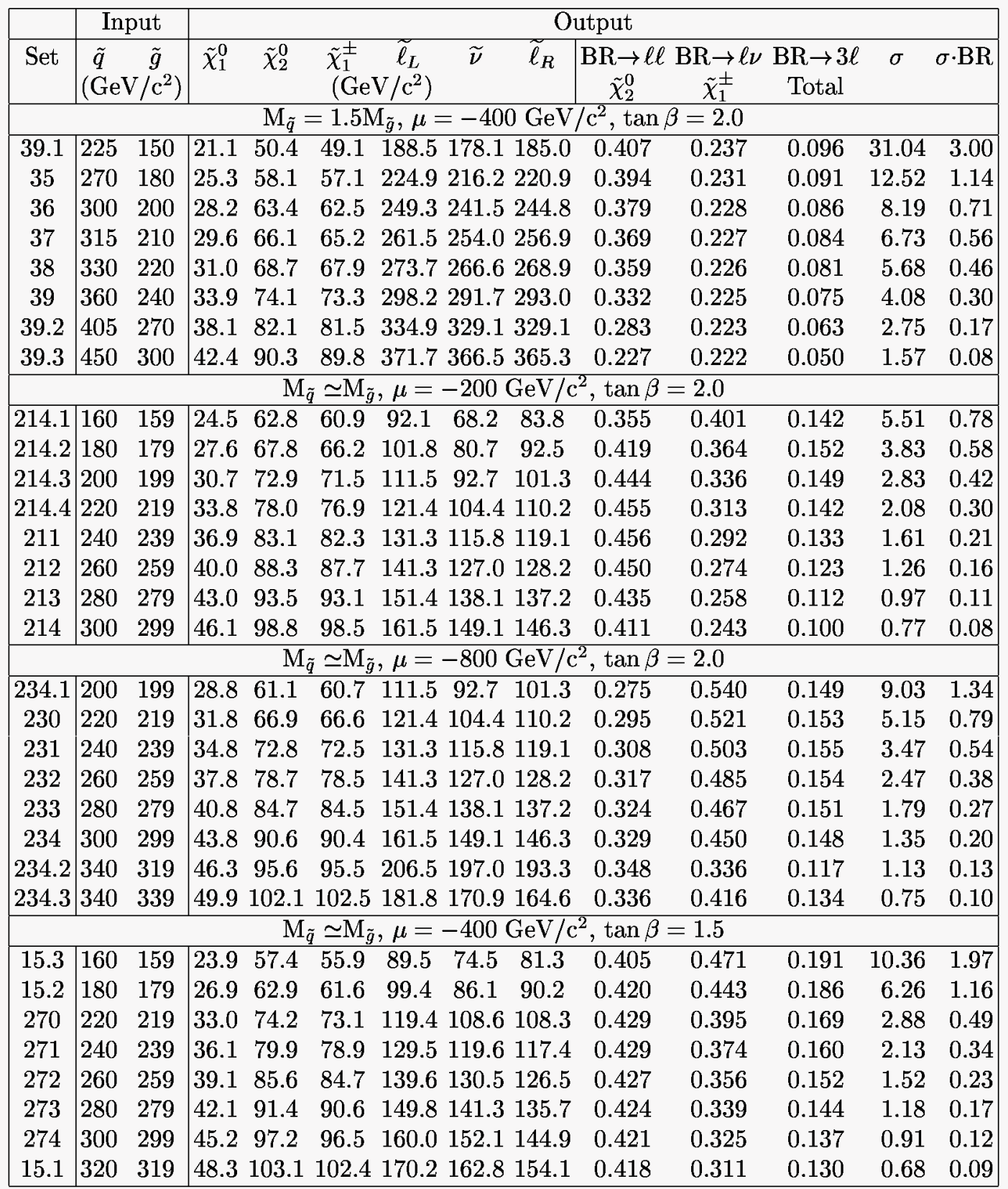


Table 5.4: Input and output parameters for the SiMSSM points scanned. All masses are in $\mathrm{GeV} / \mathrm{c}^{2}$ and all $\sigma$ are in picobarns.

\begin{tabular}{|c|c|c|c|c|c|c|c|c|c|c|c|c|c|}
\hline & \multicolumn{2}{|c|}{ Input } & \multicolumn{11}{|c|}{ Output } \\
\hline Set & $\begin{array}{c}\tilde{q} \\
(\mathrm{GeI}\end{array}$ & $\begin{array}{c}\tilde{g} \\
\left.V / c^{2}\right)\end{array}$ & $\tilde{\chi}_{1}^{0}$ & $\tilde{\chi}_{2}^{0}$ & $\begin{array}{l}\tilde{\chi}_{1}^{ \pm} \\
(\mathrm{Ge})\end{array}$ & $\begin{array}{c}\tilde{\ell}_{L} \\
\left.\mathrm{~V} / \mathrm{c}^{2}\right)\end{array}$ & $\widetilde{\nu}$ & & $\begin{array}{r}\mathrm{BR} \rightarrow \\
\tilde{\chi}_{2}^{0}\end{array}$ & $\begin{array}{l}3 \mathrm{R} \rightarrow \\
\tilde{\chi}_{1}^{ \pm}\end{array}$ & $\begin{array}{c}\mathrm{BR} \rightarrow 3 \ell \\
\text { Total }\end{array}$ & $\sigma$ & $\sigma \cdot \mathrm{BR}$ \\
\hline \multicolumn{14}{|c|}{ High $\mathrm{M}_{\tilde{q}}\left(\mathrm{M}_{\tilde{q}}=500\right) \mathrm{GeV} / \mathrm{c}^{2}, \mu=-400 \mathrm{GeV} / \mathrm{c}^{2}, \tan \beta=1.5$} \\
\hline 277 & 500 & 200 & 26.6 & 62.4 & 61.0 & 471.3 & 467.2 & 469.0 & 0.261 & 0.222 & 0.058 & 10.47 & 0.61 \\
\hline 278 & 500 & 220 & 29.7 & 68.0 & 66.8 & 464.2 & 461.6 & 461.5 & 0.255 & 0.222 & 0.057 & 6.79 & 0.39 \\
\hline 279 & 500 & 240 & 32.7 & 73.7 & 72.6 & 457.0 & 454.3 & 453.7 & 0.250 & 0.222 & 0.056 & 4.57 & 0.2 \\
\hline \multicolumn{14}{|c|}{$\mathrm{M}_{\tilde{q}} \simeq \mathrm{M}_{\tilde{g}}, \mu=-400 \mathrm{GeV} / \mathrm{c}^{2}, \tan \beta=1.1$} \\
\hline 11.4 & 160 & 159 & 24.0 & 58.8 & 57.1 & 85.8 & 82.2 & 77.9 & 0.517 & 0.476 & 246 & 8.89 & 2.19 \\
\hline 11.3 & 180 & 179 & 27.0 & 64.3 & 62.8 & 96.0 & 92.8 & 87.1 & 0.507 & 0.448 & 0.227 & 5.58 & 1.27 \\
\hline 11.2 & 200 & 199 & 30.1 & 69.9 & 68.5 & 106.3 & 103.4 & 96.4 & 0.496 & 0.422 & 0.209 & 3.85 & 0.81 \\
\hline 280 & 220 & 219 & 33.1 & 75.5 & 74.3 & 116.6 & 114.0 & 105.7 & 0.488 & 0.398 & 0.194 & 2.86 & 0.56 \\
\hline 281 & 240 & 239 & 36.2 & 81.2 & 80.1 & 126.9 & 124.5 & 115.0 & 0.478 & 0.377 & 0.180 & 2.12 & 0.38 \\
\hline 282 & 260 & 259 & 39.3 & 87.0 & 85.9 & 137.3 & 13 & 124.3 & 0.469 & 0.359 & 0.168 & 1.51 & 0.25 \\
\hline 283 & 280 & 279 & 42.3 & 92.7 & 91.8 & 147.6 & 145.5 & 133.7 & 0.457 & 0.342 & 0.156 & 1.08 & 0.17 \\
\hline 284 & 300 & 299 & 45.4 & 98.6 & 97.7 & 158.0 & 156.0 & 143.0 & 0.446 & 0.328 & 0.146 & 0.83 & 0.12 \\
\hline 11.1 & 320 & 319 & 48.4 & 104.4 & 103.6 & 168.3 & 166.5 & 152.3 & 0.434 & 0.315 & 0.137 & 0.64 & 0.09 \\
\hline \multicolumn{14}{|c|}{$\mathrm{M}_{\tilde{q}} \simeq \mathrm{M}_{\tilde{g}}, \mu=-1000 \mathrm{GeV} / \mathrm{c}^{2}, \tan \beta=2$} \\
\hline $1 \mathrm{k} .2$ & 220 & 219 & 31.5 & 65.7 & 65.5 & 121.3 & 104.3 & 110.1 & 0.278 & 0.554 & 0.154 & 5.72 & 0.88 \\
\hline $1 \mathrm{k} .3$ & 240 & 239 & 34.5 & 71.6 & 71.5 & 131.3 & 115.7 & 119.1 & 0.293 & 0.538 & 0.157 & 3.87 & 0.61 \\
\hline $1 \mathrm{k} .4$ & 260 & 259 & 37.5 & 77.6 & 77.4 & 141.3 & 127.0 & 128.1 & 0.303 & 0.522 & 0.158 & 2.64 & 0.41 \\
\hline $1 \mathrm{k} .5$ & 280 & 279 & 40.5 & 83.6 & 83.4 & 151.4 & 138.1 & 137.2 & 0.311 & 0.506 & 0.157 & 1.85 & 0.29 \\
\hline $1 \mathrm{k} .6$ & 300 & 299 & 43.5 & 89.6 & 89.4 & 161.5 & 149.1 & 146.3 & 0.316 & 0.490 & 0.155 & 1.41 & 0.21 \\
\hline $1 \mathrm{k} .7$ & 320 & 319 & 46.5 & 95.6 & 95.4 & 171.6 & 160.0 & 155.4 & 0.321 & 0.474 & 0.152 & 1.05 & 0.16 \\
\hline $1 \mathrm{k} .8$ & 340 & 339 & 49.6 & 101.6 & 101.5 & 181.8 & 170.9 & 164.6 & 0.324 & 0.458 & 0.148 & 0.77 & 0.11 \\
\hline \multicolumn{14}{|c|}{$\mathrm{M}_{\tilde{q}} \simeq \mathrm{M}_{\tilde{g}}, \mu=-600 \mathrm{GeV} / \mathrm{c}^{2}, \tan \beta=2$} \\
\hline 0.1 & 200 & 199 & \begin{tabular}{|l|}
29.1 \\
\end{tabular} & 62.9 & 62.4 & 111.5 & 92.7 & 101.2 & 0.301 & 0.496 & 0.149 & 6.79 & 1.02 \\
\hline .2 & 220 & 219 & 32.1 & 68.7 & 68.2 & 121.3 & 104.3 & 110.1 & 0.319 & 0.474 & 0.151 & 4.54 & 0.68 \\
\hline 3 & 240 & 239 & 35.1 & 74.5 & 74.1 & 131.3 & 115.7 & 119.1 & 0.331 & 0.453 & 0.150 & 3.10 & 0.46 \\
\hline 4 & 260 & 259 & 38.1 & 80.4 & 80.0 & 141.3 & 127.0 & 128.1 & 0.339 & 0.433 & 0.147 & 2.16 & 0.31 \\
\hline & 280 & 279 & 41.1 & 86.3 & 85.9 & 151.4 & 138.1 & 137.2 & 0.345 & 0.415 & 0.143 & 1.56 & 0.22 \\
\hline & 300 & 299 & 44.2 & 92.2 & 91.9 & 161.5 & 149.1 & 146.3 & 0.348 & 0.397 & 0.138 & 1.19 & 0.16 \\
\hline & 320 & 319 & 47.2 & 98.2 & 97.9 & 171.6 & 160.0 & 155.4 & 0.351 & 0.380 & 0.133 & 0.90 & 0.12 \\
\hline 600.8 & 10 & 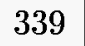 & 50.3 & 104.1 & 103.9 & 181.0 & 160.9 & 4.6 & 353 & 0.364 & 0.128 & 0.68 & 0.08 \\
\hline
\end{tabular}


Table 5.5: Input and output parameters for the SiMSSM points scanned. All masses are in $\mathrm{GeV} / \mathrm{c}^{2}$ and all $\sigma$ are in picobarns. Note that the $\tilde{\chi}_{2}^{0}$ branching ratio is quite small for the cases where the $\tilde{q}$ is lighter than the $\tilde{g}$.

\begin{tabular}{|c|c|c|c|c|c|c|c|c|c|c|c|c|c|}
\hline \multirow{3}{*}{\begin{tabular}{|l} 
Set \\
\end{tabular}} & \multicolumn{2}{|c|}{ Input } & \multicolumn{11}{|c|}{ Output } \\
\hline & \multirow{2}{*}{\multicolumn{2}{|c|}{$\begin{array}{|cc|}\tilde{q} & \tilde{g} \\
\left(\mathrm{GeV} / \mathrm{c}^{2}\right) \\
\end{array}$}} & \multirow[t]{3}{*}{$\tilde{\chi}_{1}^{0}$} & & \multirow{2}{*}{\multicolumn{2}{|c|}{$\begin{array}{cc}\tilde{\chi}_{1}^{ \pm} & \widetilde{\ell}_{L} \\
\left(\mathrm{GeV} / \mathrm{c}^{2}\right)\end{array}$}} & \multirow[t]{2}{*}{$\widetilde{u}$} & & \multirow{2}{*}{\multicolumn{3}{|c|}{$\begin{array}{ccc}\mathrm{BR} \rightarrow \ell \ell & \mathrm{BR} \rightarrow \ell \nu & \mathrm{BR} \rightarrow 3 \ell \\
\tilde{\chi}_{2}^{0} & \tilde{\chi}_{1}^{ \pm} & \text {Total } \\
\end{array}$}} & \multirow[t]{2}{*}{$\sigma$} & \multirow[t]{2}{*}{$\sigma \cdot \mathrm{BR}$} \\
\hline & & & & & & & & & & & & & \\
\hline \multicolumn{12}{|c|}{$\mathrm{M}_{\tilde{q}}=2 \mathrm{M}_{\tilde{g}}, \mu=-400 \mathrm{GeV} / \mathrm{c}^{2}, \tan \beta=2$} & & \\
\hline 20.1 & 300 & 150 & 20.3 & 48.9 & 47.7 & 273.7 & 266.6 & 271.2 & 0.359 & 0.224 & 0.080 & 39.52 & 3.18 \\
\hline 20.2 & 340 & 170 & 23.0 & 53.9 & 52.8 & 309.5 & 303.3 & 306.9 & 0.336 & 0.223 & 0.074 & 21.38 & 1.60 \\
\hline 20.3 & 380 & 190 & 25.7 & 58.9 & 57.9 & 345.5 & 339.9 & 342.6 & 0.302 & 0.222 & 0.067 & 13.15 & 0.88 \\
\hline 20.4 & 420 & 210 & 28.5 & 64.0 & 63.1 & 381.5 & 376.4 & 378.3 & 0.259 & 0.222 & 0.057 & 8.63 & 0.49 \\
\hline 20.5 & 460 & 230 & 31.2 & 69.1 & 68.3 & 417.5 & 412.9 & 414.1 & 0.213 & 0.221 & 47 & 6.04 & 0.28 \\
\hline 20.6 & 500 & 250 & 34.0 & 74.3 & 73.5 & 453.5 & 449.3 & 449.8 & 0.168 & 0.221 & 7 & 4.39 & 0.16 \\
\hline 20.7 & 540 & 270 & 36.7 & 79.5 & 78.8 & 489.6 & 485.6 & 485.6 & 0.128 & 0.221 & 0.028 & 3.27 & 0.09 \\
\hline \multicolumn{14}{|c|}{$\mathrm{M}_{\tilde{q}} \sim \mathrm{M}_{\tilde{g}}, \mu=-400 \mathrm{GeV} / \mathrm{c}^{2}, \tan \beta=4$} \\
\hline 4.8 & 160 & 159 & 23.0 & 49.2 & 48.9 & 95.5 & 59.0 & 87.0 & 0.090 & 0.606 & & 29.56 & 1.62 \\
\hline 4.2 & 200 & 199 & 28.9 & 60.4 & 60.2 & 114.3 & 86.2 & 103.9 & 0.204 & 0.539 & & 8.44 & 0.93 \\
\hline 4.3 & 220 & 219 & 31.8 & 66.1 & 65.9 & 123.9 & 98.6 & 112.6 & 0.237 & 0.514 & 0.122 & 5.56 & 0.68 \\
\hline 4.4 & 240 & 239 & 34.8 & 71.8 & 71.7 & 133.7 & 110.6 & 121.4 & 0.261 & 0.492 & 0.128 & 3.74 & 0.48 \\
\hline 4.5 & 260 & 259 & 37.8 & 77.6 & 77.5 & 143.6 & 122.3 & 130.2 & 0.278 & 0.471 & 0.131 & 2.58 & 0.34 \\
\hline 4.6 & 280 & 279 & 40.8 & 83.4 & 83.3 & 153.5 & 133.8 & 139.2 & 0.290 & 0.450 & 31 & 1.84 & 0.24 \\
\hline 4.7 & 300 & 299 & 43.8 & 89.2 & 89.1 & 163.5 & 145.1 & 148.2 & 0.298 & 0.429 & & 1.40 & 0.18 \\
\hline 4.10 & 320 & 319 & 46.8 & 95.0 & 955.0 & 173.5 & 156.3 & 157.2 & 0.304 & 0.408 & & 1.06 & 0.13 \\
\hline 4.11 & 340 & 339 & 49.8 & 100.9 & 100.9 & 183.5 & 167.4 & 166.3 & 0.306 & 0.387 & 0.118 & 0.80 & 0.10 \\
\hline \multicolumn{14}{|c|}{$\mathrm{M}_{\tilde{q}} \sim \mathrm{M}_{\tilde{g}}, \mu=-400 \mathrm{GeV} / \mathrm{c}^{2}, \tan \beta=8$} \\
\hline 8.1 & 180 & 179 & 25.9 & 51.0 & 51.0 & 105.8 & 70.6 & 96.2 & 0.100 & 0.637 & 0.064 & 23.54 & 1.51 \\
\hline 8.2 & 200 & 199 & 28.1 & 56.7 & 56.7 & 115.2 & 84.0 & 104.7 & 0.144 & 0.627 & 0.090 & 12.45 & 1.12 \\
\hline 8.3 & 220 & 219 & 31.0 & 62.4 & 62.4 & 124.8 & 96.7 & 113.3 & 0.178 & 0.616 & 10 & 7.58 & 0.83 \\
\hline 8.5 & 260 & 259 & 36.9 & 73.8 & 73.9 & 144.2 & 120.8 & 130.9 & 0.224 & 0.596 & 0.133 & 3.34 & 0.45 \\
\hline 8.6 & 280 & 279 & 39.9 & 79.6 & 79.7 & 154.1 & 132.5 & 139.8 & 0.238 & 0.584 & 0.139 & 2.34 & 0.33 \\
\hline 8.7 & 300 & 299 & 42.9 & 85.4 & 85.5 & 164.0 & 143.9 & 148.7 & 0.249 & 0.570 & 0.142 & 1.75 & 0.25 \\
\hline 8.9 & 320 & 319 & 45.9 & 91.3 & 91.3 & 174.0 & 155.2 & 157.7 & 0.256 & 0.552 & 0.141 & 1.30 & 0.18 \\
\hline 8.10 & 340 & 339 & 48.9 & 97.1 & 97.1 & 184.1 & 166.4 & 166.8 & 0.260 & 0.530 & 0.138 & 0.97 & 0.13 \\
\hline \multicolumn{14}{|c|}{$\mathrm{M}_{\tilde{q}}=0.9 \mathrm{M}_{\tilde{g}}, \mu=-400 \mathrm{GeV} / \mathrm{c}^{2}, \tan \beta=2$} \\
\hline 2 & 180 & 200 & 30.6 & 67.8 & 67.0 & 67.4 & 26.6 & 48.5 & 0.006 & 0.667 & 0.004 & 4.03 & 0.01 \\
\hline 3 & 198 & 220 & 33.6 & 73.6 & 72.9 & 72.2 & 37.1 & 51.0 & 0.008 & 0.667 & 0.005 & 2.80 & 0.01 \\
\hline 4 & 216 & 240 & 36.7 & 79.5 & 78.8 & 77.2 & 46.0 & 53.6 & 0.011 & 0.667 & 0.007 & 1.93 & 0.01 \\
\hline 5 & 234 & 260 & 39.8 & 85.3 & 84.7 & 82.2 & 54.0 & 56.3 & 0.016 & 0.667 & 0.010 & 1.38 & 0.01 \\
\hline 6 & 252 & 280 & 42.9 & 91.3 & 90.7 & 87.3 & 61.5 & 59.2 & 0.022 & 0.667 & 0.014 & 1.05 & 0.02 \\
\hline 7 & 270 & 300 & 46.0 & 97.2 & 96.7 & 92.4 & 68.6 & 62.0 & 0.028 & 0.667 & 0.019 & 0.80 & 0.02 \\
\hline
\end{tabular}


Table 5.6: Input and output parameters for the SiMSSM points scanned. All masses are in $\mathrm{GeV} / \mathrm{c}^{2}$ and all $\sigma$ are in picobarns.

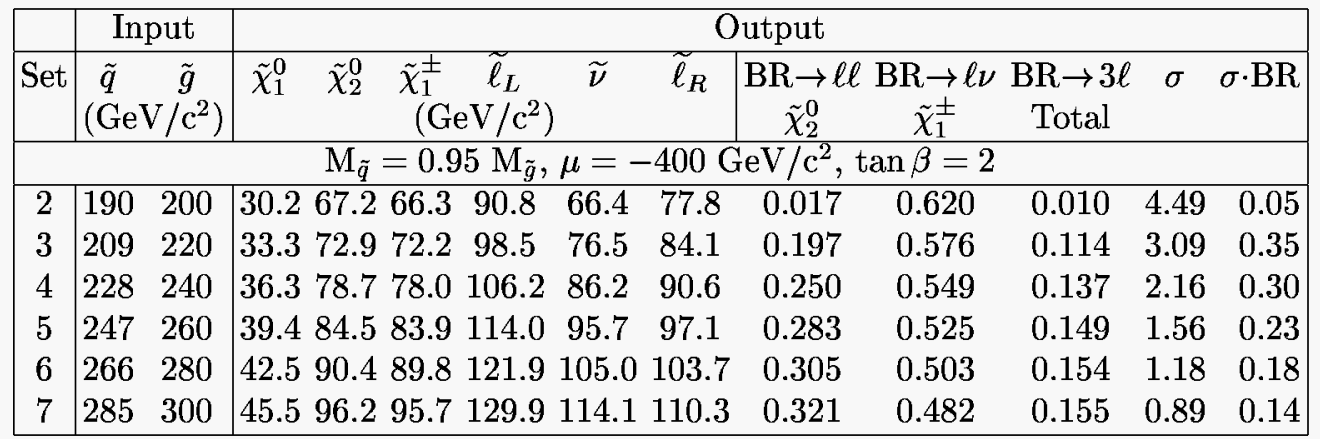

1. a top quark mass of $\approx 175 \mathrm{GeV} / \mathrm{c}^{2}$;

2. light $\tilde{\chi}_{1}^{ \pm}$and $\tilde{\chi}_{2}^{0}: \mathrm{M}_{\tilde{\chi}_{1}^{ \pm}} \approx \mathrm{M}_{\tilde{\chi}_{2}^{0}} \leq 90 \mathrm{GeV} / \mathrm{c}^{2}$

3. light $\widetilde{\ell}_{R}: \mathrm{M}_{\widetilde{\ell}_{R}} \approx 45-50 \mathrm{GeV} / \mathrm{c}^{2}<\mathrm{M}_{\tilde{\chi}_{2}^{0}}<\mathrm{M}_{\widetilde{\ell}_{L}}$

4. $\mathrm{M}_{\tilde{q}} \approx 0.98 \mathrm{M}_{\tilde{g}}$.

In this model, the trilepton signal is nearly maximized because the $\tilde{\chi}_{2}^{0}$ decays exclusively to dileptons with dominant two-body decay modes $\tilde{\chi}_{2}^{0} \rightarrow \ell^{ \pm} \widetilde{\ell}_{R}^{\mp}(B R=66 \%$ for $e$ and $\mu)$ and $\widetilde{\ell}_{R} \rightarrow \ell \tilde{\chi}_{1}^{0}(B R=100 \%)$. This results in a final state with three leptons and $\mathscr{E}_{T}$ (Figures 2.18 and 2.20). Since we observe zero trilepton events, we set limits on this model using ISAJET ${ }^{9}$ with parameters supplied by J. Lopez (as listed in Table 5.7) [48].

Figure 5.17 shows the acceptance for this model as a function of $\tilde{\chi}_{1}^{ \pm}$mass. The acceptance decreases for large $\tilde{\chi}_{1}^{ \pm}$masses as the $\tilde{\chi}_{1}^{0}$ mass approaches the $\tilde{\ell}_{R}$ mass (see Table 5.8) and carries away much of the energy, resulting in soft leptons. As shown in Figure 5.18, we exclude

$$
\begin{aligned}
M_{\tilde{\chi}_{1}^{ \pm}} & <80.5 \mathrm{GeV} / \mathrm{c}^{2} \\
\sigma_{\tilde{\chi}_{1}^{ \pm} \tilde{\chi}_{2}^{0}} \cdot B R\left(\tilde{\chi}_{1}^{ \pm} \tilde{\chi}_{2}^{0} \rightarrow 3 \ell+X\right) & >0.4 \mathrm{pb} .
\end{aligned}
$$

This is comparable to the ALEPH limit of $83 \mathrm{GeV} / \mathrm{c}^{2}$ [47]. We do not entirely exclude the $\mathrm{SU}(5) \times \mathrm{U}(1)$ Supergravity model. However, our analysis excludes the model for small and moderate $\tilde{\chi}_{1}^{ \pm}$masses and leaves only a small window for $\tilde{\chi}_{1}^{ \pm} \mathrm{s}$ heavier than $80.5 \mathrm{GeV} / \mathrm{c}^{2}$.

\footnotetext{
${ }^{9}$ We use ISAJET 7.06 for this section of this analysis. It does not have explicit Supergravity input parameters. However, J. Lopez calculated the necessary input parameters for use with ISAJET 7.06.
} 


\section{Search for SUSY using $\bar{p} \bar{p} \rightarrow$ eee, ee $\mu, e \mu \mu, \mu \mu \mu$}

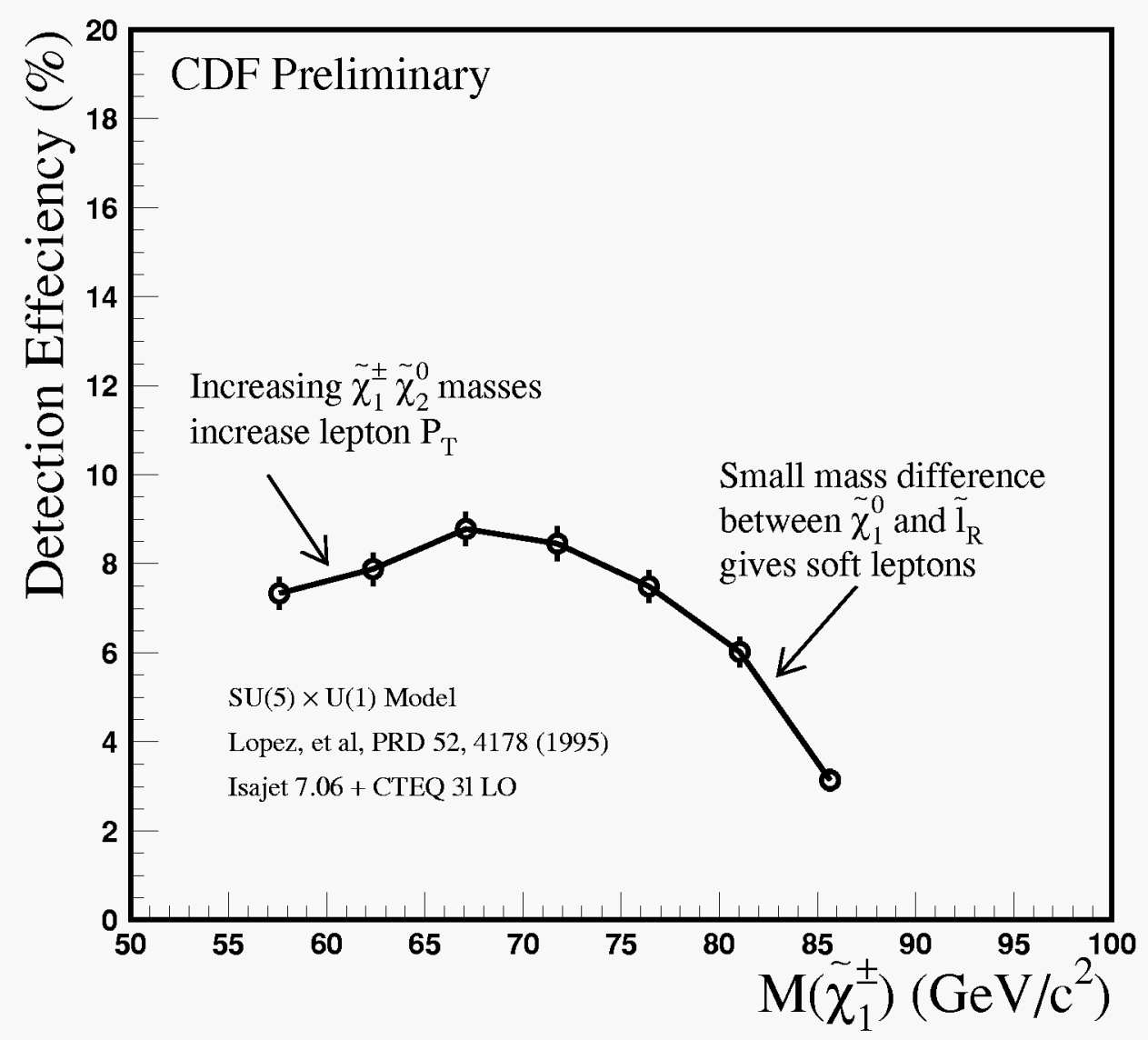

Figure 5.17: The acceptance for all four trilepton modes for $\mathrm{SU}(5) \times \mathrm{U}(1)$ model production of trileptons. The acceptance decreases for large $\tilde{\chi}_{1}^{ \pm}$masses as the $\tilde{\chi}_{1}^{0}$ mass approaches the $\tilde{\ell}_{R}$ mass and carries away much of the energy, resulting in soft leptons. The error bars indicate the statistical uncertainty. 


\section{Search for SUSY using $p \bar{p} \rightarrow$ eee, ee $\mu, e \mu \mu, \mu \mu \mu$}

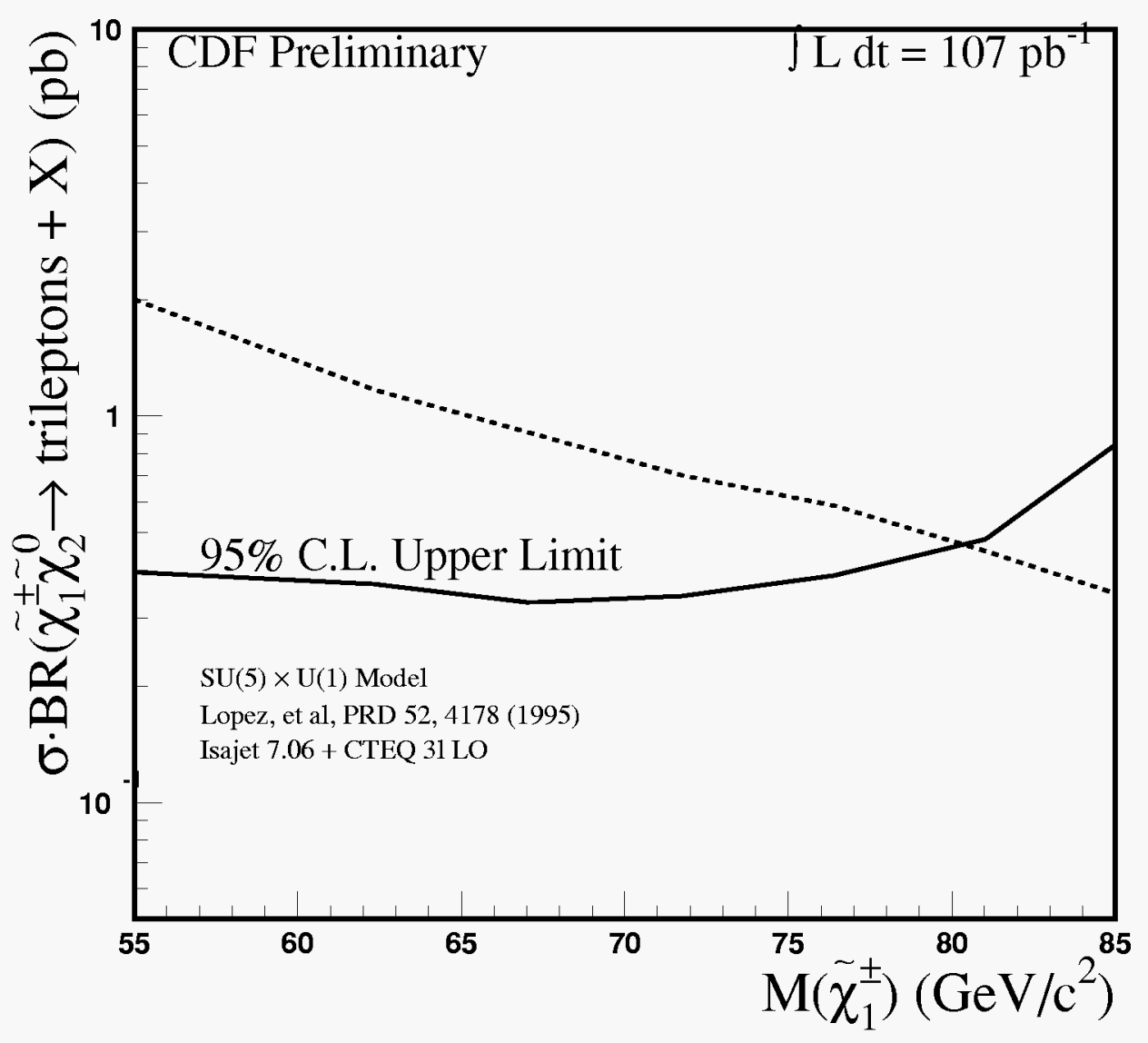

Figure 5.18: $\sigma \cdot B R$ versus $\tilde{\chi}_{1}^{ \pm}$mass for $\mathrm{SU}(5) \times \mathrm{U}(1)$ model production of trileptons. This sets a mass limit of $80.5 \mathrm{GeV} / \mathrm{c}^{2}$ and a cross section times branching ratio limit of $0.4 \mathrm{pb}$. 
Table 5.7: The input and output parameters used to examine the $\mathrm{SU}(5) \times \mathrm{U}(1)$ model. These values are courtesy of Jorge Lopez. Note that $\tan \beta=1.4$ for the entire set and all masses are in $\mathrm{GeV} / \mathrm{c}^{2}$.

\begin{tabular}{|c|c|c|c|c|c|c|c|c|c|c|c|}
\hline $\mathrm{M}_{\tilde{g}}$ & $\mathrm{M}_{\tilde{q}}$ & $\mathrm{M}_{\tilde{\ell}_{L}}$ & $\mathrm{M}_{\tilde{\ell}_{R}}$ & $\mathrm{M}_{\tilde{\nu}_{L}}$ & $\mathrm{M}_{\tilde{t}_{L}}$ & $\mathrm{M}_{\tilde{t}_{R}}$ & $\mathrm{~A}_{t}$ & $\mathrm{M}_{\tilde{b}_{L}}$ & $\mathrm{M}_{\tilde{b}_{R}}$ & $\mu$ & $\mathrm{M}_{H_{A}}$ \\
\hline 310.2 & 302.9 & 107.1 & 44.4 & 85.2 & 197.0 & 394.6 & -211.7 & 328.7 & 308.9 & 242.3 & 295.0 \\
\hline 322.9 & 315.6 & 111.0 & 45.1 & 89.9 & 205.0 & 405.5 & -220.4 & 342.0 & 321.4 & 252.2 & 306.6 \\
\hline 335.7 & 328.3 & 114.8 & 45.8 & 94.6 & 213.1 & 416.5 & -229.1 & 355.4 & 333.9 & 262.1 & 318.3 \\
\hline 348.4 & 341.0 & 118.7 & 46.6 & 99.2 & 221.4 & 427.5 & -237.8 & 368.7 & 346.5 & 271.9 & 329.8 \\
\hline 361.2 & 353.6 & 122.7 & 47.3 & 103.8 & 229.7 & 438.5 & -246.5 & 382.1 & 359.0 & 281.7 & 341.5 \\
\hline 373.9 & 366.3 & 126.6 & 48.1 & 108.3 & 238.3 & 449.6 & -255.2 & 395.5 & 371.5 & 291.3 & 352.8 \\
\hline 386.6 & 378.9 & 130.5 & 48.8 & 112.9 & 246.8 & 460.7 & -263.9 & 408.9 & 384.1 & 301.0 & 364.3 \\
\hline
\end{tabular}

Table 5.8: Masses of the $\tilde{\chi}_{1}^{ \pm}, \tilde{\chi}_{2}^{0}, \tilde{\chi}_{1}^{0}$ for the points examined in the $\mathrm{SU}(5) \times \mathrm{U}(1)$ model. For comparison, the difference in masses between the $\tilde{\chi}_{1}^{ \pm}$and the $\widetilde{\ell}_{R}$ and the detection efficiency are shown. We have reasonable efficiency as long as the mass difference is $\geq 5 \mathrm{GeV} / \mathrm{c}^{2}$. All masses are in $\mathrm{GeV} / \mathrm{c}^{2}$ and the efficiency is in \%.

\begin{tabular}{|c|r|r|r|r|r|}
\hline $\mathrm{M}_{\tilde{g}}$ & $\mathrm{M}_{\tilde{\chi}_{1}^{ \pm}}$ & $\mathrm{M}_{\tilde{\chi}_{2}^{0}}$ & $\mathrm{M}_{\tilde{\chi}_{1}^{0}}$ & $\mathrm{M}_{\tilde{\ell}_{R}}-\mathrm{M}_{\tilde{\chi}_{1}^{0}}$ & efficiency \\
\hline 310.2 & 57.5 & 67.0 & 29.1 & 15.2 & 7.3 \\
322.9 & 62.3 & 70.8 & 32.1 & 12.9 & 7.9 \\
335.7 & 67.0 & 74.7 & 35.0 & 10.7 & 8.8 \\
348.4 & 71.7 & 78.6 & 37.8 & 8.7 & 8.5 \\
361.2 & 76.4 & 82.6 & 40.6 & 6.7 & 7.5 \\
373.9 & 81.0 & 86.7 & 43.2 & 4.8 & 6.0 \\
386.6 & 85.6 & 90.8 & 45.8 & 3.0 & 3.1 \\
\hline
\end{tabular}

\subsubsection{SUGRA}

The SiMSSM as we have examined it only unifies the electromagnetic, weak and strong forces. Supergravity (or SUGRA) [49] also unifies gravity with the other three forces [13, 14]. SUGRA has a nice feature in that gauge symmetry-breaking is induced at the weak scale as a requirement of the model. However, it is a more constrained model than the SiMSSM so limits set using SUGRA are not as general. It has four and one-half free parameters: $m_{0}$, the common scalar mass; $m_{1 / 2}$, the common gaugino mass; the sign of $\mu ; \tan \beta$; and $A_{t}$. As seen in Section 5.3, the trilepton analysis strongly depends on the squark to gluino mass ratio and $\mu$. These parameters are not free in the context of SUGRA which makes it more difficult to understand a limit at a particular point in SUGRA space.

SUGRA is currently quite popular, so, despite its limitations, we have examined several 
points in SUGRA space. Figure 5.19 shows the detection efficiency as a function of $\mathrm{M}_{\tilde{\chi}_{1}^{ \pm}}$. Since the squark to gluino mass ratio and $\mu$ are now set inside the model, it is impossible to plot limits as a function of them. Therefore, we plot many different theoretical $\sigma_{\tilde{\chi}_{1}^{ \pm} \tilde{\chi}_{2}^{0}} \cdot \operatorname{BR}\left(\tilde{\chi}_{1}^{ \pm} \tilde{\chi}_{2}^{0} \rightarrow 3 \ell+X\right)$ points on the same plot for comparison with a single 95\% C.L. limit curve in Figure 5.20. As before, we use ISAJET 7.20 with the CTEQ31 parton distribution function. The values of $\mu$ tend to be rather low, and as seen in Figure 5.9 that degrades our limit setting ability. We set $\tan \beta=2, \mathrm{~A}=0$ and require $\mu<0$. We exclude

$$
\begin{aligned}
M_{\tilde{\chi}_{1}^{ \pm}} & <62 \mathrm{GeV} / \mathrm{c}^{2} \\
\sigma_{\tilde{\chi}_{1}^{ \pm} \tilde{\chi}_{2}^{0}} \cdot B R\left(\tilde{\chi}_{1}^{ \pm} \tilde{\chi}_{2}^{0} \rightarrow 3 \ell+X\right) & >0.5 \mathrm{pb} .
\end{aligned}
$$

Tables 5.9-5.13 list the input and output parameters used. 


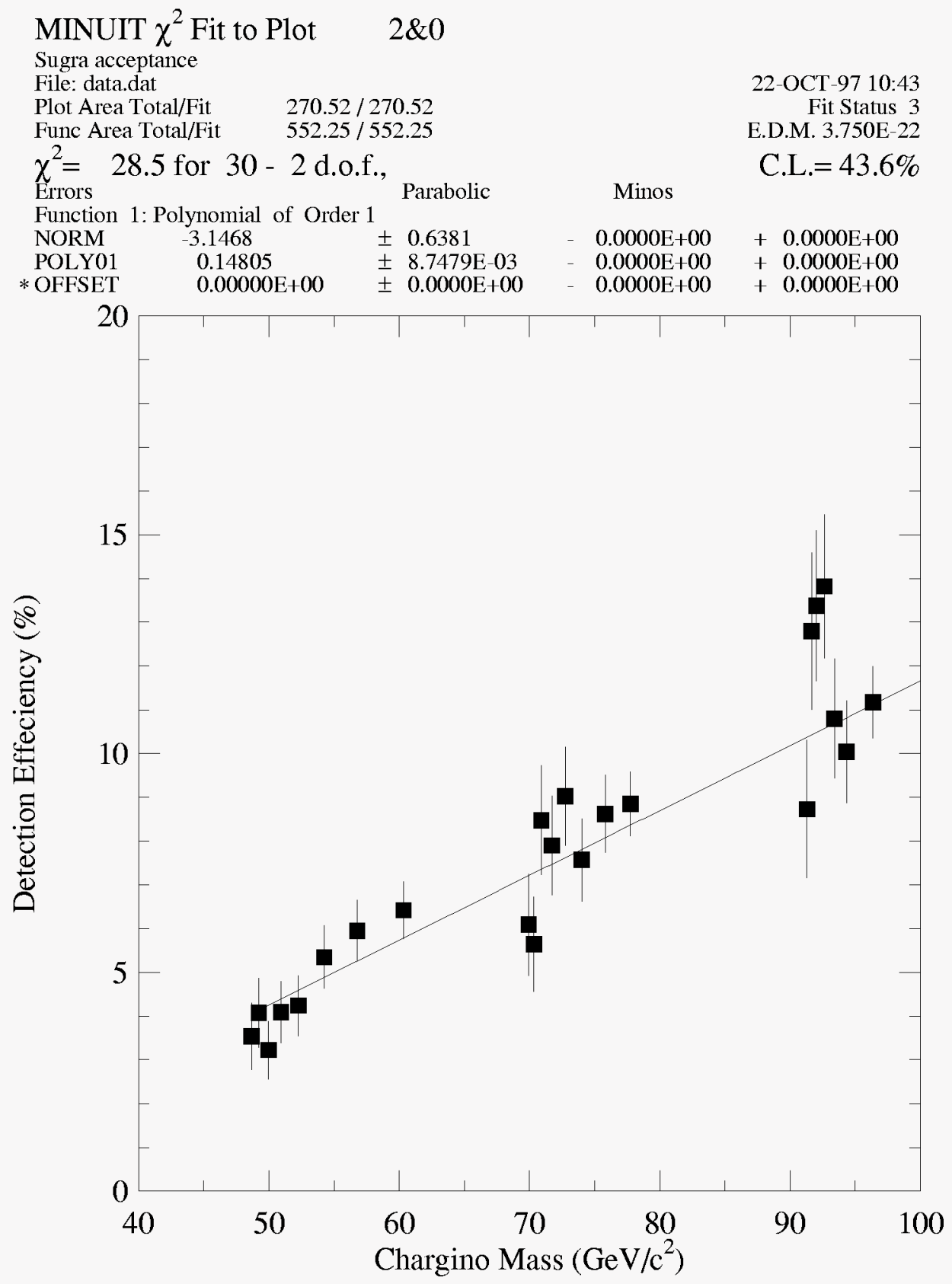

Figure 5.19: Detection efficiency as a function of $\mathrm{M}_{\tilde{\chi}_{1}^{ \pm}}$in the framework of the SUGRA model. 


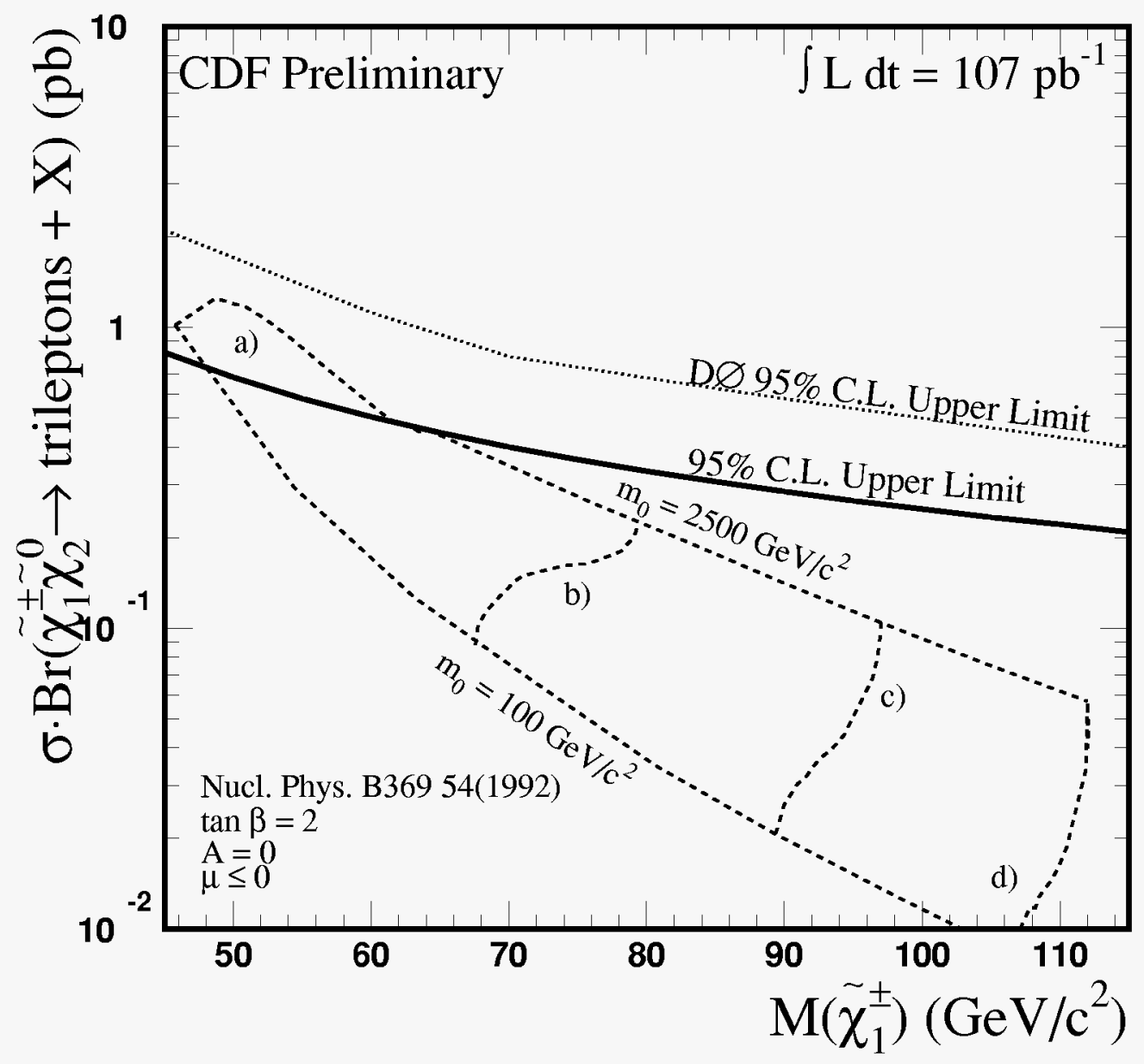

Figure 5.20: Production cross section times branching ratio to leptons for $\tilde{\chi}_{1}^{ \pm} \tilde{\chi}_{2}^{0}$ production as a function of $\tilde{\chi}_{1}^{ \pm}$mass within the SUGRA framework (dashed lines). The solid line is the 95\% C.L. limit curve; all points above it are excluded. We set $\tan \beta=2, \mathrm{~A}=0$ and require $\mu \leq 0$. For lines a)-d) we set $100 \mathrm{GeV} / \mathrm{c}^{2} \leq \mathrm{m}_{0} \leq 2500 \mathrm{GeV} / \mathrm{c}^{2}$ and (a) $\mathrm{m}_{1 / 2}=50 \mathrm{GeV} / \mathrm{c}^{2}$, (b) $\mathrm{m}_{1 / 2}=75 \mathrm{GeV} / \mathrm{c}^{2}$, (c) $\mathrm{m}_{1 / 2}=100 \mathrm{GeV} / \mathrm{c}^{2}$ and (d) $\mathrm{m}_{1 / 2}=120 \mathrm{GeV} / \mathrm{c}^{2}$. For the lines $\mathrm{m}_{0}=100 \mathrm{GeV} / \mathrm{c}^{2}$ and 2500 $\mathrm{GeV} / \mathrm{c}^{2}$ we set $50 \leq \mathrm{m}_{1 / 2} \leq 120 \mathrm{GeV} / \mathrm{c}^{2}$. For comparison we show the D0 $95 \%$ C.L. limit curve (dotted line). 
Table 5.9: Input and output parameters for the SUGRA points scanned. All masses are in $\mathrm{GeV} / \mathrm{c}^{2}$. $\mathrm{m}_{0}$ and $\mathrm{m}_{1 / 2}$ are input parameters and $\mu$ is an output parameter.

\begin{tabular}{|c|ccc||c|ccc|}
\hline Set & $\mathrm{m}_{0}$ & $\mathrm{~m}_{1 / 2}$ & $\mu$ & Set & $\mathrm{m}_{0}$ & $\mathrm{~m}_{1 / 2}$ & $\mu$ \\
\hline 1 & 200 & 50 & -186.069 & $\mathrm{~b} 1$ & 100 & 75 & -163.662 \\
2 & 300 & 50 & -266.678 & $\mathrm{~b} 2$ & 120 & 75 & -172.144 \\
3 & 400 & 50 & -350.862 & $\mathrm{~b} 3$ & 140 & 75 & -183.200 \\
4 & 500 & 50 & -444.768 & $\mathrm{~b} 4$ & 160 & 75 & -194.698 \\
5 & 600 & 50 & -536.425 & $\mathrm{~b} 5$ & 180 & 75 & -205.839 \\
6 & 700 & 50 & -623.811 & $\mathrm{~b} 6$ & 1000 & 75 & -893.558 \\
7 & 800 & 50 & -714.489 & $\mathrm{~b} 7$ & 1500 & 75 & -1345.179 \\
8 & 900 & 50 & -805.791 & $\mathrm{~b} 8$ & 2000 & 75 & -1813.768 \\
9 & 200 & 75 & -219.498 & $\mathrm{~b} 9$ & 2500 & 75 & -2240.462 \\
10 & 300 & 75 & -291.085 & $\mathrm{c} 1$ & 100 & 100 & -207.422 \\
11 & 400 & 75 & -371.920 & $\mathrm{c} 2$ & 120 & 100 & -214.530 \\
12 & 500 & 75 & -456.418 & $\mathrm{c} 3$ & 140 & 100 & -222.741 \\
13 & 600 & 75 & -542.042 & $\mathrm{c} 4$ & 160 & 100 & -231.186 \\
14 & 700 & 75 & -630.116 & $\mathrm{c} 5$ & 180 & 100 & -241.346 \\
15 & 800 & 75 & -716.663 & $\mathrm{c} 6$ & 1000 & 100 & -880.959 \\
16 & 900 & 75 & -803.768 & $\mathrm{c} 7$ & 1500 & 100 & -1315.166 \\
17 & 200 & 100 & -252.082 & $\mathrm{c} 8$ & 2000 & 100 & -1750.734 \\
19 & 400 & 100 & -386.463 & $\mathrm{c} 9$ & 2500 & 100 & -2216.317 \\
20 & 500 & 100 & -464.421 & $\mathrm{~d} 1$ & 100 & 120 & -241.032 \\
21 & 600 & 100 & -545.403 & $\mathrm{~d} 2$ & 120 & 120 & -246.732 \\
22 & 700 & 100 & -627.902 & $\mathrm{~d} 3$ & 140 & 120 & -253.473 \\
23 & 800 & 100 & -709.089 & $\mathrm{~d} 4$ & 160 & 120 & -261.335 \\
24 & 900 & 100 & -794.251 & $\mathrm{~d} 5$ & 180 & 120 & -269.867 \\
25 & 200 & 120 & -279.473 & $\mathrm{~d} 6$ & 1000 & 120 & -886.271 \\
26 & 300 & 120 & -335.698 & $\mathrm{~d} 7$ & 1500 & 120 & -1313.595 \\
27 & 400 & 120 & -404.963 & $\mathrm{~d} 8$ & 2000 & 120 & -1760.341 \\
28 & 500 & 120 & -476.957 & $\mathrm{~d} 9$ & 2500 & 120 & -2198.631 \\
29 & 600 & 120 & -552.079 & $\mathrm{e} 1$ & 2500 & 40 & -2273.547 \\
30 & 700 & 120 & -633.072 & $\mathrm{e} 2$ & 2500 & 50 & -2274.281 \\
31 & 800 & 120 & -714.718 & $\mathrm{e} 3$ & 2500 & 60 & -2271.083 \\
32 & 900 & 120 & -794.095 & $\mathrm{e} 4$ & 2500 & 70 & -2244.072 \\
$\mathrm{a} 1$ & 180 & 50 & -170.656 & $\mathrm{e} 5$ & 2500 & 80 & -2259.290 \\
$\mathrm{a} 2$ & 160 & 50 & -156.111 & $\mathrm{e} 6$ & 2500 & 90 & -2209.621 \\
$\mathrm{a} 3$ & 140 & 50 & -144.023 & $\mathrm{e} 7$ & 2500 & 100 & -2216.317 \\
$\mathrm{a} 4$ & 120 & 50 & -129.635 & $\mathrm{e} 8$ & 2500 & 110 & -2213.630 \\
$\mathrm{a} 5$ & 100 & 50 & -117.714 & $\mathrm{e} 9$ & 2500 & 120 & -2198.631 \\
\hline
\end{tabular}


Table 5.10: Input and output parameters for the SUGRA points scanned. All masses are in $\mathrm{GeV} / \mathrm{c}^{2}$. $\mathrm{m}_{0}$ and $\mathrm{m}_{1 / 2}$ are input parameters and $\mu$ is an output parameter.

\begin{tabular}{|c|ccc||c|ccc|}
\hline Set & $\mathrm{m}_{0}$ & $\mathrm{~m}_{1 / 2}$ & $\mu$ & Set & $\mathrm{m}_{0}$ & $\mathrm{~m}_{1 / 2}$ & $\mu$ \\
\hline $\mathrm{f} 1$ & 100 & 40 & -99.201 & $\mathrm{f} 6$ & 100 & 90 & -189.670 \\
$\mathrm{f} 2$ & 100 & 50 & -117.714 & $\mathrm{f} 7$ & 100 & 100 & -207.422 \\
$\mathrm{f3}$ & 100 & 60 & -135.514 & $\mathrm{f} 8$ & 100 & 110 & -224.225 \\
$\mathrm{f} 4$ & 100 & 70 & -153.708 & $\mathrm{f} 9$ & 100 & 120 & -241.032 \\
$\mathrm{f5}$ & 100 & 80 & -172.995 & & & & \\
\hline
\end{tabular}


Table 5.11: Output parameters for the SUGRA points scanned. All $\sigma$ are in picobarns.

\begin{tabular}{|c|c|c|c|c|c|c|c|c|c|c|c|c|c|}
\hline Set & $\tilde{q}$ & $\tilde{g}$ & $\tilde{\chi}_{1}^{0}$ & $\begin{array}{l}\tilde{\chi}_{2}^{0} \\
(\mathrm{Ge}\end{array}$ & $\begin{array}{c}\tilde{\chi}_{1}^{ \pm} \\
\left.V / c^{2}\right) \\
\end{array}$ & $\widetilde{\ell}_{L}$ & $\widetilde{\nu}$ & $\widetilde{\ell}_{R}$ & $\begin{array}{c}\mathrm{BR} \rightarrow \ell \ell \\
\tilde{\chi}_{2}^{0}\end{array}$ & $\begin{array}{c}\mathrm{BR} \rightarrow \ell \nu \\
\tilde{\chi}_{1}^{ \pm}\end{array}$ & $\begin{array}{c}\mathrm{BR} \rightarrow 3 \ell \\
\text { Total }\end{array}$ & $\sigma$ & $\sigma \cdot \mathrm{BR}$ \\
\hline 1 & 237.3 & 168.0 & 23.5 & 62.3 & 60.3 & 206.5 & 197.0 & 203.8 & 0.33 & 0.22 & 0.07 & 7.55 & 0.54 \\
\hline 2 & 324.3 & 173.1 & 23.2 & 58.5 & 56.8 & 304.4 & 298.0 & 302.5 & 0.26 & 0.22 & 0.06 & 12.85 & 0.73 \\
\hline 3 & 417.8 & 177.1 & 23.0 & 55.5 & 54.2 & 403.3 & 398.5 & 401.9 & 0.22 & 0.22 & 0.05 & 18.87 & 0.92 \\
\hline 4 & 513.6 & 179.2 & 22.8 & 53.2 & 52.3 & 502.6 & 498.8 & 501.5 & 0.19 & 0.22 & 0.04 & 24.78 & 1.07 \\
\hline 5 & 611.0 & 181.3 & 22.7 & 51.7 & 50.9 & 602.2 & 599.0 & 601.3 & 0.17 & 0.22 & 0.04 & 30.26 & 1.17 \\
\hline 6 & 709.2 & 183.5 & 22.6 & 50.6 & 50.0 & 701.8 & 699.1 & 701.1 & 0.16 & 0.22 & 0.03 & 34.66 & 1.20 \\
\hline 7 & 807.9 & 185.5 & 22.5 & 49.7 & 49.2 & 801.6 & 799.2 & 801.0 & 0.14 & 0.22 & 0.03 & 38.88 & 1.23 \\
\hline 8 & 906.9 & 188.0 & 22.4 & 49.0 & 48.6 & 901.4 & 899.3 & 900.8 & 0.13 & 0.22 & 0.03 & 42.84 & 1.24 \\
\hline 9 & 275.0 & 236.5 & 34.1 & 78.9 & 77.8 & 210.4 & 201.1 & 204.9 & 0.34 & 0.22 & 0.07 & 2.37 & 0.18 \\
\hline 10 & 351.0 & 240.6 & 33.9 & 76.8 & 75.8 & 307.0 & 300.7 & 303.3 & 0.23 & 0.22 & 0.05 & 3.24 & 0.16 \\
\hline 11 & 438.6 & 246.8 & 33.6 & 74.8 & 74.0 & 405.2 & 400.5 & 402.5 & 0.18 & 0.22 & 0.04 & 4.09 & 0.16 \\
\hline 12 & 530.2 & 252.0 & 33.4 & 73.3 & 72.7 & 504.2 & 500.4 & 502.0 & 0.15 & 0.22 & 0.03 & 4.77 & 0.16 \\
\hline 13 & 624.8 & 255.9 & 33.2 & 72.2 & 71.7 & 603.5 & 600.3 & 601.7 & 0.13 & 0.22 & 0.03 & 5.36 & 0.15 \\
\hline 14 & 720.7 & 258.9 & 33.1 & 71.3 & 70.9 & 703.0 & 700.2 & 701.4 & 0.11 & 0.22 & 0.03 & 5.84 & 0.15 \\
\hline 15 & 1.9 & 262.0 & 33.0 & 70.7 & 70.3 & 802.6 & 800.2 & 801.2 & 0.10 & 0.22 & 0.02 & 6.19 & 0.14 \\
\hline 16 & 915.3 & 261.6 & 33.0 & 70.2 & 69.9 & 902.3 & 900.2 & 901.1 & 0.10 & 0.22 & 0.02 & 6.39 & 0.14 \\
\hline 17 & 316.7 & 298.4 & $=44.5$ & 97.0 & 96.4 & 215.7 & 206.6 & 206.5 & 0.33 & 0.22 & 0.07 & 0.92 & 0.07 \\
\hline 19 & 465.9 & 314.2 & 44.0 & 94.8 & 94.3 & 408.0 & 403.2 & 403.3 & 0.15 & 0.22 & 0.03 & 1.34 & 0.04 \\
\hline 20 & 552.4 & 319.1 & 43.9 & 93.8 & 93.4 & 506.4 & 502.6 & 502.6 & 0.12 & 0.22 & 0.03 & 1.52 & 0.04 \\
\hline 21 & 642.9 & 32.3 .4 & $=43.7$ & 93.0 & 92.6 & 605.3 & 60 & 602.2 & 0.10 & 0.22 & 0.02 & 1.67 & 0.04 \\
\hline 22 & 0.4 & 327.7 & 43.6 & 92.3 & 92.0 & 704.5 & 70 & 701.9 & 0.09 & 0.22 & 0.02 & 1.78 & 0.03 \\
\hline 23 & 830.8 & 327 & 43.7 & 91.9 & 91.7 & 803.9 & 80 & 801.6 & 0.08 & 0.22 & 0.02 & 1.85 & 0.03 \\
\hline 24 & 926.8 & 330.7 & 43.6 & 91.5 & 91.3 & 903.5 & 90 & 901.5 & 0.07 & 0.22 & 0.02 & 1.93 & 0.03 \\
\hline 25 & 352.2 & 346.8 & 52.8 & 112.3 & 111.9 & 220.9 & 212.0 & 208.1 & 0.33 & 0.23 & 0.07 & 0.46 & 0.03 \\
\hline 27 & 489.8 & 36 & 52.4 & 110.8 & 110.5 & 410.7 & 406 & 404.1 & 0.14 & 0.22 & 0.03 & 0.63 & 0.02 \\
\hline 28 & 571.3 & 364 & 52.4 & 110.2 & 109.9 & 508.5 & 50 & 503.3 & 0.10 & 0.22 & 0.02 & 0.71 & 0.02 \\
\hline 29 & 659.9 & 374 & 52.2 & 109.6 & 109.3 & 607.1 & 603.9 & 602.7 & 0.08 & 0.22 & 0.02 & 0.77 & 0.01 \\
\hline 30 & 750.6 & 378.6 & 52.1 & 109.0 & 108.8 & 706.1 & 703.3 & 702.3 & 0.07 & 0.22 & 0.02 & 0.83 & 0.01 \\
\hline 31 & 844.0 & 379 & $=52.0$ & 108.7 & 108.5 & 805.3 & 802.9 & 802.1 & 0.07 & 0.22 & 0.01 & 0.86 & 0.01 \\
\hline 32 & 938.1 & 382.9 & 52.1 & 108.4 & 108.2 & 904.7 & 902.6 & 901.8 & 0.06 & 0.22 & 0.01 & 0.88 & 0.01 \\
\hline a1 & 220.8 & 166.8 & 23.5 & 63.1 & 61.2 & 187.2 & 176.7 & 184.2 & 0.35 & 0.22 & 0.08 & 6.64 & 0.50 \\
\hline $\mathrm{a} 2$ & 205.6 & 165.5 & 23.6 & 63.8 & 62.0 & 168.1 & 15 & 164.7 & 0.37 & 0.22 & 0.08 & 5.89 & 0.48 \\
\hline a3 & 191.5 & 164.5 & 23.6 & 64.1 & 62.6 & 149.2 & 13 & 145.4 & 0.41 & 0.22 & 0.09 & 5.30 & 0.48 \\
\hline $\mathrm{a} 4$ & 178.0 & 163.3 & 23.6 & 64.3 & 63.4 & 130.6 & 115.0 & 126.2 & 0.44 & 0.22 & 0.10 & 4.55 & 0.45 \\
\hline a5 & 166.0 & 161.8 & 23.7 & 64.1 & 64.1 & 112.6 & 94.0 & 107.4 & 0.48 & 0.23 & 0.11 & 4.07 & 0.46 \\
\hline
\end{tabular}


Table 5.12: Output parameters for the SUGRA points scanned. All $\sigma$ are in picobarns.

\begin{tabular}{|c|c|c|c|c|c|c|c|c|c|c|c|c|c|}
\hline Set & $\tilde{q}$ & $\tilde{g}$ & $\tilde{\chi}_{1}^{0}$ & $\tilde{\chi}_{2}^{0}$ & $\begin{array}{c}\tilde{\chi}_{1}^{ \pm} \\
\mathrm{GeV} / \mathrm{c}\end{array}$ & $\widetilde{\ell}_{L}$ & $\widetilde{\nu}$ & $\ell_{R}$ & $\begin{array}{c}\mathrm{BR} \rightarrow \ell \\
\tilde{\chi}_{2}^{0}\end{array}$ & $\begin{array}{c}\mathrm{BR} \rightarrow \ell \\
\tilde{\chi}_{1}^{ \pm}\end{array}$ & $\begin{array}{c}\mathrm{BR} \rightarrow 3 \ell \\
\text { Total }\end{array}$ & $\sigma$ & $\sigma \cdot \mathrm{BR}$ \\
\hline b1 & 218 & 230 & 34.3 & 79.9 & 79.4 & 119.7 & 102.4 & 109.6 & 0.46 & 0.29 & 0.13 & 1.68 & 0.22 \\
\hline $\mathrm{b} 2$ & 226 & 229 & 34.3 & 79.9 & 79.2 & 136.8 & 122.0 & 128.1 & 0.46 & 0.25 & 0.12 & 1.78 & 0.21 \\
\hline b3 & 237 & 232 & 34.2 & 79.8 & 78.9 & 154.6 & 141.7 & 147.0 & 0.44 & 0.24 & 0.10 & 1.91 & 0.20 \\
\hline b4 & 248 & 233 & 34.2 & 79.5 & 78.5 & 172.9 & 161.4 & 166.1 & 0.40 & 0.23 & 0.09 & 2.06 & 0.19 \\
\hline $\mathrm{b} 5$ & 261 & 233 & 34.2 & 79.2 & 78.2 & 191.5 & 181.2 & 185.5 & 0.37 & 0.22 & 0.08 & 2.21 & 0.18 \\
\hline $\mathrm{b} 6$ & 1013 & 264 & 33.0 & 69.8 & 69.5 & 1002.1 & 1000.1 & 1001.0 & 0.09 & 0.22 & 0.02 & .63 & 0.13 \\
\hline b7 & 1508 & 272 & 32.8 & 68.5 & 68.4 & 1501.4 & 1500.1 & 1500.7 & 0.07 & 0.22 & 0.02 & 7.39 & 0.11 \\
\hline b8 & 2006 & 275 & 32.7 & 67.8 & 67.8 & 2001.0 & 2000.0 & 2000.5 & 0.06 & 0.22 & 0.01 & 7.73 & 0.10 \\
\hline b9 & 2504 & 284 & 32.7 & 67.6 & 67.6 & 2500.8 & 2500.0 & 2500.4 & 0.05 & 0.22 & 0.01 & 7.86 & 0.09 \\
\hline c1 & 269 & 291 & 44.7 & 97.4 & 97.0 & 128.9 & 113.0 & 112.6 & 0.40 & 0.36 & 0.14 & .74 & 0.10 \\
\hline $\mathrm{c} 2$ & 276 & 293 & 44.6 & 97.4 & 97.0 & 144.9 & 131.0 & 130.6 & .42 & 0.29 & 0.12 & 0.77 & 0.09 \\
\hline c3 & 285 & 294 & 44.6 & 97.4 & 96.8 & 161.8 & 149.4 & 149.2 & .41 & 0.26 & 0.11 & .80 & 0.08 \\
\hline $\mathrm{c} 4$ & 294 & 295 & 44.6 & 97.3 & 96.7 & 179.3 & 168.3 & 168.1 & .39 & 0.2 & 0.09 & .84 & 0.08 \\
\hline c5 & 305 & 297 & 44.6 & 97.2 & 96.6 & 197.3 & 187.4 & 187.2 & 0.36 & 0.23 & 0.08 & 88 & 0.07 \\
\hline c6 & 1023 & 334 & 43.6 & 91.1 & 90.9 & 1003.1 & 1001.2 & 1001.3 & 0.07 & 0.2 & 0.02 & 01 & 0.03 \\
\hline c7 & 151 & 344 & 43.4 & 90.1 & 90.0 & 1502.1 & 1500.8 & 1500.9 & 0.0 & 0.2 & 0.01 & 17 & .03 \\
\hline c8 & 201 & 353 & 43.4 & 89.7 & 89.6 & 200 & 20 & 2000.7 & 0.0 & 0.2 & 0.01 & 2.24 & 0.02 \\
\hline c9 & 2508 & 355 & 43.5 & 89.4 & 89.4 & 2501.2 & 2500.4 & 2500.5 & 0.04 & 0.2 & 0.01 & 2.25 & 0.02 \\
\hline $\mathrm{d} 1$ & 310 & 340 & 52.9 & 112.3 & 112.0 & 137.4 & 122.6 & 115.5 & 0.36 & 0.4 & 0.15 & 0.39 & 0.06 \\
\hline $\mathrm{d} 2$ & 316 & 342 & 52.9 & 112.4 & 112.1 & 152.5 & 139.4 & 133.2 & 0.39 & 0.3 & 0.12 & 0.40 & 0.05 \\
\hline $\mathrm{d} 3$ & 324 & 3 & 52.9 & 112.4 & 112.1 & 168.6 & 156.8 & 151.4 & 0.39 & 0.2 & 0. & 0.41 & 0.04 \\
\hline $\mathrm{d} 4$ & 332 & 344 & 52.9 & 112.4 & 112.0 & 185.5 & 174.9 & 170.1 & 0.38 & 0.2 & 0. & 0.43 & 0.04 \\
\hline $\mathrm{d} 5$ & 342 & 345 & 52.9 & 112.3 & 112.0 & 203.0 & 193.3 & 189.0 & 0.35 & 0.2 & 0. & 0.44 & 0.04 \\
\hline $\mathrm{d} 6$ & 1033 & 384 & 52.0 & 108.0 & 107.8 & 1004.2 & 1002.3 & 1001.6 & 0.06 & 0.2 & 0 . & 0.92 & 0.01 \\
\hline $\mathrm{d} 7$ & 1521 & 0 & 52.0 & 107.2 & 107.1 & 1502.8 & 1501. & 1501.1 & 0.05 & 0.2 & 0 . & 0.99 & 0.01 \\
\hline $\mathrm{d} 8$ & 2015 & 406 & 52.1 & 106.9 & 106.8 & 2002.1 & 2001. & 2000.8 & 0.04 & 0.2 & 0.01 & 1.03 & 0.01 \\
\hline e1 & 2501 & 168 & 17.7 & 37.1 & 37.1 & 2500 . & 2499. & 2500.3 & 0.07 & 0.22 & 0.02 & 3482 & 55.0 \\
\hline $\mathrm{e} 2$ & 2502 & 20 & 22.0 & 45.8 & 45.8 & 2500.5 & 2499. & 2500.3 & 0.06 & 0.2 & 0.01 & 2.02 & 1.02 \\
\hline e3 & 2502 & 234 & 26.3 & 54.5 & 54.5 & 2500.6 & 2499. & 2500.3 & 0.06 & 0.22 & 0.01 & 22.62 & 0.29 \\
\hline e4 & 2504 & 26 & 30.6 & 63.2 & 63.2 & 2500.7 & 2500.0 & 2500.4 & 0.05 & 0.22 & 0.01 & 10.81 & 0.13 \\
\hline e 5 & 2505 & 29 & 34.9 & 71.9 & 71.9 & 2500.9 & 250 & 2500.4 & 0.05 & 0.22 & 0.01 & 5.99 & 0.07 \\
\hline e6 & 250 & 32 & 39.1 & 80.7 & 80.6 & 2501.0 & 250 & 2500.5 & 0.0 & 0.2 & 0. & 3.58 & 0.03 \\
\hline e7 & 2508 & 35 & 43.5 & 89.4 & 89.4 & 2501.2 & 250 & 2500.5 & 0.0 & 0.22 & 0.01 & 2.25 & 0.02 \\
\hline $\mathrm{e} 8$ & 2509 & 38 & 47.8 & 98.2 & 98.1 & 250 & 250 & 2500.6 & 0.0 & 0.22 & 0.01 & 1.50 & 0.01 \\
\hline $\mathrm{e} 9$ & 2511 & 411 & 52 & 106.7 & 106.7 & 250 & 2500.9 & 2500.7 & 0.04 & 0.22 & 0.01 & 1.05 & 0.01 \\
\hline
\end{tabular}


Table 5.13: Output parameters for the SUGRA points scanned. All $\sigma$ are in picobarns.

\begin{tabular}{|c|c|c|c|c|c|c|c|c|c|c|c|c|c|}
\hline Set & $\tilde{q}$ & $\tilde{g}$ & $\tilde{\chi}_{1}^{0}$ & $\begin{array}{l}\tilde{\chi}_{2}^{0} \\
(\mathrm{GeI})\end{array}$ & $\begin{array}{c}\tilde{\chi}_{1}^{ \pm} \\
\left.V / \mathrm{c}^{2}\right)\end{array}$ & $\widetilde{\ell}_{L}$ & $\widetilde{\nu}$ & $\widetilde{\ell}_{R}$ & $\begin{array}{r}\mathrm{BR} \rightarrow \\
\tilde{\chi}_{2}^{0} \\
\end{array}$ & $\begin{array}{c}\mathrm{R} \rightarrow \ell \nu \\
\tilde{\chi}_{1}^{ \pm}\end{array}$ & $\begin{array}{c}\mathrm{BR} \rightarrow 3 \ell \\
\text { Total }\end{array}$ & $\sigma$ & B \\
\hline f1 & 15 & & 19.2 & 57.9 & 58.9 & 110.5 & 91.5 & & 045 & 0.21 & & & \\
\hline $\mathrm{f} 2$ & & & 23.7 & 64.1 & 64 & & & & & & & & \\
\hline $\mathrm{f} 3$ & & & Or & 1.3 & 69.9 & & & & & & & & \\
\hline $\mathrm{f} 4$ & & & & 0.7 & 76.2 & & & & & & & & \\
\hline f5 & & & & & 82.8 & & & & & & & & \\
\hline f6 & & & & 90.2 & 89.8 & & & & & & & & \\
\hline f7 & & & 44 & 97.4 & 97.0 & & & & & & & & \\
\hline f8 & & & & 048 & 10 & & & & & & & & 0.0 \\
\hline f9 & 0.8 & 340. & 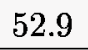 & 112.8 & 2.0 & 7. & 122.6 & 115.5 & 0.36 & 0.41 & 0.15 & 0.39 & 0.0 \\
\hline
\end{tabular}




\section{Chapter 6}

\section{Conclusion}

We have searched for evidence of Supersymmetry using the CDF detector and the Fermilab Tevatron. After searching $107 \mathrm{pb}^{-1}$ for three lepton events, we find no evidence for $\tilde{\chi}_{1}^{ \pm} \tilde{\chi}_{2}^{0}$ production.

We set limits on $\mathrm{M}_{\tilde{\chi}_{1}^{ \pm}}$and $\sigma_{\tilde{\chi}_{1}^{ \pm} \tilde{\chi}_{2}^{0}} \cdot \operatorname{BR}\left(\tilde{\chi}_{1}^{ \pm} \tilde{\chi}_{2}^{0} \rightarrow 3 \ell+X\right)$ in several models. Table 6.1 lists the limits for a general MSSM, Table 6.2 lists the limit for the $\mathrm{SU}(5) \times \mathrm{U}(1)$ supergravity model, and Table 6.3 lists the limits for a general SUGRA model.

In Section 2.5 I introduced the concept of naturalness. In Figure 2.21 we see the naturalness of the $\tilde{\chi}_{1}^{ \pm}$. The limit set by this analysis is well away from the minimum in that plot and begins to approach non-natural (or unlikely) values. Also, if the model assumptions we make are correct, we see that the naturalness of the squark, gluino and slepton masses are starting to move away from their most natural values (see Figure 6.1). We are beginning to challenge SUSY.

It is appropriate to wonder what reach the Tevatron will have during Run II. At high gluino mass the best search mode is $\tilde{\chi}_{1}^{ \pm} \tilde{\chi}_{2}^{0}$ or $\tilde{\chi}_{1}^{ \pm} \tilde{\chi}_{1}^{ \pm}$: the production cross section for gluinos and squarks becomes quite small (as seen in Figure 6.2), while the $\tilde{\chi}_{1}^{ \pm}$and $\tilde{\chi}_{2}^{0}$ masses tend to be $\sim 1 / 3$ the gluino masses. Table 6.4 lists the upper reach for CDF during Run II, for either 1 or $2 \mathrm{fb}^{-1}$.

Either Supersymmetry will be discovered during Run II at the Tevatron or its viability as a physically acceptable theory will be greatly compromised. 


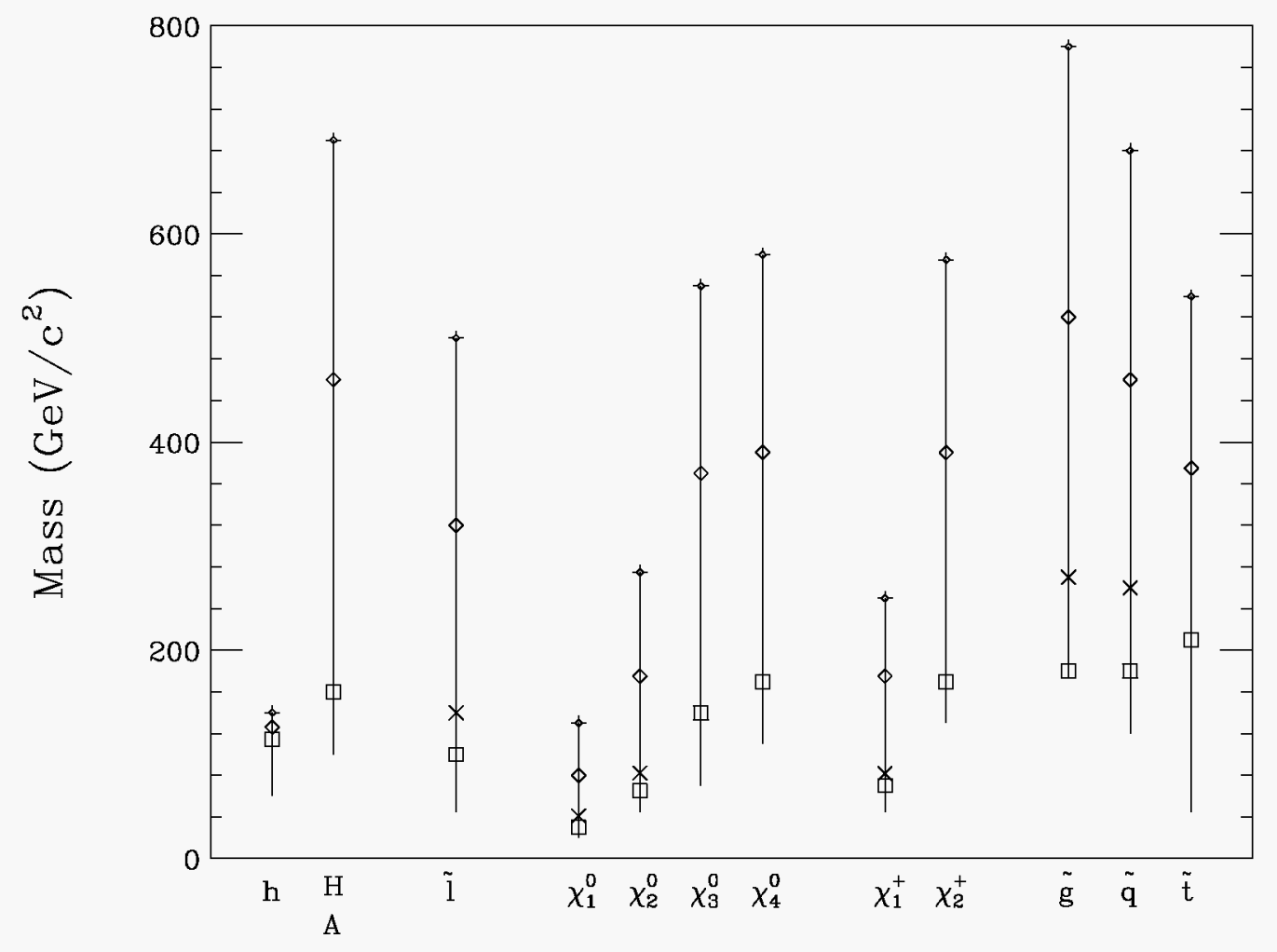

Figure 6.1: The naturalness for a variety of different sparticles. The bottom of each line is the world limit prior to this analysis, the box $(\square)$ represents a naturalness of 1 , the diamond $(\diamond)$ a naturalness of 5 and the top of the line $(+)$ a naturalness of 10. The limits from this analysis (primarily for $\tilde{\chi}_{1}^{ \pm}$and $\tilde{\chi}_{2}^{0}$, but also for $\tilde{\ell}, \tilde{g}$ and $\tilde{q}$ ) are represented by the cross $(\times)$. We have excluded the most natural values. Adapted from Ref. [25]. 
Table 6.1: Limits on chargino and neutralino masses within the framework of the SUGRA-inspired MSSM. All masses are in $\mathrm{GeV} / \mathrm{c}^{2}$.

\begin{tabular}{|c|c|c|c||c|c|c|c|}
\hline \multicolumn{3}{|c||}{ Input Parameters } & \multicolumn{5}{c|}{ Limits } \\
\hline $\mathrm{M}_{\tilde{g}}$ & $\mathrm{M}_{\tilde{q}}$ & $\tan \beta$ & $\mu$ & $\mathrm{M}_{\tilde{\chi}_{1}^{ \pm}}$ & $\mathrm{M}_{\tilde{\chi}_{2}^{0}}$ & $\mathrm{M}_{\tilde{\chi}_{1}^{0}}$ & $\sigma \cdot \mathrm{BR}(\mathrm{pb})$ \\
\hline 220 & 330 & 2 & -400 & 68.0 & 69.0 & 29.2 & 0.45 \\
220 & 264 & 2 & -400 & 71.0 & 72.1 & 32.0 & 0.40 \\
200 & 200 & 2 & -200 & 72.5 & 73.4 & 30.7 & 0.40 \\
240 & 240 & 2 & -400 & 76.5 & 77.1 & 36.1 & 0.37 \\
260 & 260 & 2 & -600 & 81.5 & 82.2 & 38.1 & 0.34 \\
270 & 270 & 2 & -800 & 81.0 & 81.0 & 39.3 & 0.35 \\
260 & 260 & 2 & -1000 & 78.5 & 78.4 & 37.5 & 0.36 \\
200 & 500 & 2 & -400 & 58.0 & 59.0 & 26.4 & 0.60 \\
\hline
\end{tabular}

Table 6.2: Limits on chargino and neutralino masses within the framework of the $\mathrm{SU}(5) \times \mathrm{U}(1)$ model. All masses are in $\mathrm{GeV} / \mathrm{c}^{2}$. Recall for this model $\tan \beta=1.4$.

\begin{tabular}{|c|c|c||c|c|c|c|}
\hline \multicolumn{3}{|c||}{ Input Parameters } & \multicolumn{4}{c|}{ Limits } \\
\hline $\mathrm{M}_{\tilde{g}}$ & $\mathrm{M}_{\tilde{q}}$ & $\mu$ & $\mathrm{M}_{\tilde{\chi}_{1}^{ \pm}}$ & $\mathrm{M}_{\tilde{\chi}_{2}^{0}}$ & $\mathrm{M}_{\tilde{\chi}_{1}^{0}}$ & $\sigma \cdot \mathrm{BR}(\mathrm{pb})$ \\
\hline 373.9 & 366.3 & 291.3 & 81.0 & 86.7 & 43.2 & 0.4 \\
\hline
\end{tabular}

Table 6.3: Limits on chargino and neutralino masses within the framework of SUGRA. All masses are in $\mathrm{GeV} / \mathrm{c}^{2}$. Recall we used $\tan \beta=2$ and $A=0$.

\begin{tabular}{|l|c||c|c|c|c|c|c|c|}
\hline \multicolumn{2}{|c||}{$\begin{array}{l}\text { Input } \\
\text { parameters }\end{array}$} & \multicolumn{10}{|c|}{ Limits } \\
\hline$m_{0}$ & $m_{1 / 2}$ & $\mathrm{M}_{\tilde{g}}$ & $\mathrm{M}_{\tilde{q}}$ & $\mu$ & $\mathrm{M}_{\tilde{\chi}_{1}^{ \pm}}$ & $\mathrm{M}_{\tilde{\chi}_{2}^{0}}$ & $\mathrm{M}_{\tilde{\chi}_{1}^{0}}$ & $\sigma \cdot \mathrm{BR}(\mathrm{pb})$ \\
\hline 200 & 50 & 237.3 & 168.0 & -186.1 & 62.3 & 60.3 & 23.5 & 0.5 \\
\hline
\end{tabular}



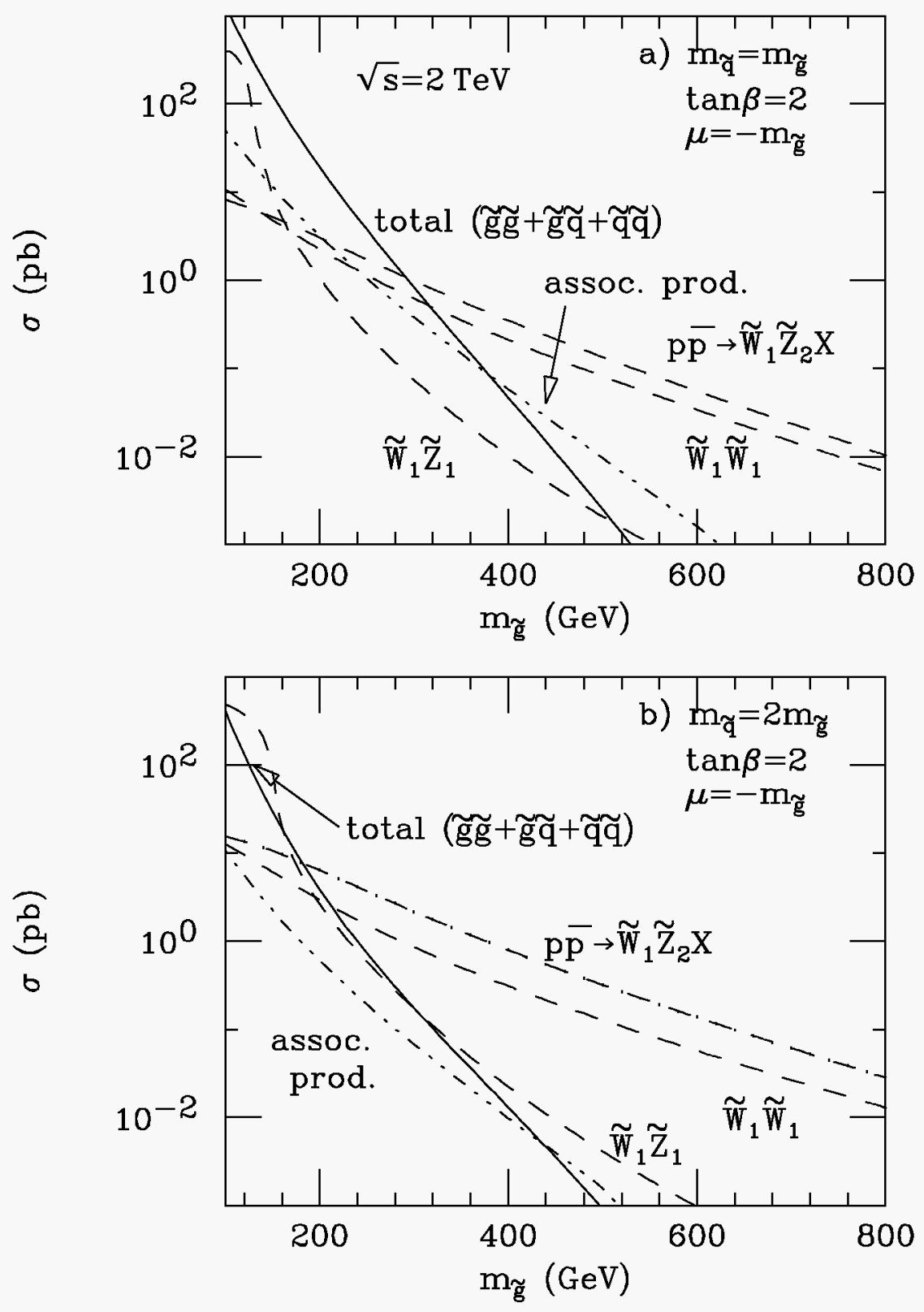

Figure 6.2: Production cross section for a variety of processes at the upgraded Tevatron. Clearly, the best search modes are $\tilde{\chi}_{1}^{ \pm} \tilde{\chi}_{2}^{0}$ or $\tilde{\chi}_{1}^{ \pm} \tilde{\chi}_{1}^{ \pm}$. Adapted from Ref. [9]. 
Table 6.4: Lower limits for several searches using Run II at the Tevatron. For the various gluino and squark searches, OS means searching for opposite sign dilepton + jets, SS is same sign dilepton + jets, $3 \ell$ is trilepton + jets, and $\not_{T}$ is $\not_{T}+$ jets. Adapted from Refs. [9] and [50].

\begin{tabular}{|c|l|r|r|}
\hline & \multicolumn{2}{|c|}{ Search } & \multicolumn{2}{|c|}{ Limit $\left(\mathrm{GeV} / \mathrm{c}^{2}\right)$} \\
Particle & Method & $1 \mathrm{fb}^{-1}$ & $2 \mathrm{fb}^{-1}$ \\
\hline$\tilde{\chi}_{1}^{ \pm} \tilde{\chi}_{2}^{0}$ & $3 \ell+\boldsymbol{E}_{T}$ & 150 & 210 \\
\hline$\tilde{g}$ and $\tilde{q}:$ & & & \\
$m_{\tilde{q}}=m_{\tilde{g}}+10 \mathrm{GeV}$ & $\not_{T}$ & 260 & \\
& $O S$ & 290 & \\
& $S S$ & 320 & \\
$m_{\tilde{q}}=m_{\tilde{g}}-10 \mathrm{GeV}$ & $3 \ell$ & 425 & \\
\cline { 2 - 4 } & $\mathscr{H}_{T}$ & 265 & \\
& $O S$ & 235 & \\
& $S S$ & 325 & \\
$m_{\tilde{q}}=2 m_{\tilde{g}}$ & $3 \ell$ & 440 & \\
\cline { 2 - 4 } & $\not_{T}$ & 200 & \\
& $O S$ & 180 & \\
& $S S$ & 210 & \\
\hline$\tilde{t}$ & $3 \ell$ & 260 & \\
\hline$\tilde{\ell}$ & all & 125 & 150 \\
\hline & all & 100 (for $\left.25 \mathrm{fb}^{-1}\right)$ \\
\hline
\end{tabular}




\section{Bibliography}

[1] Webster's New World Dictionary, $2^{\text {nd }}$ College Edition, 1984.

[2] K. G. Wilson, Phys.Rev.D10:2445-2459 (1974); G. Altarelli and G. Parisi, Nucl.Phys. B126:298 (1977).

[3] S. L. Glashow, Nucl. Phys. 22, 579-588, 1961; S. Weinberg, Phys. Rev. Lett. 19, 1264-66, 1967; A. Salam, in Elementary Particle Theory, N. Svartholm, ed. (Stockholm: Almquist and Wiksell, 1968).

[4] F. Abe, et al. (The CDF Collaboration), March 1995, Phys. Rev. Lett. 74:2626-2631,1995.

[5] Particle Data Group, Review of Particle Physics, Phys. Rev. D54, 1 July 1996.

[6] M. Drees and S. Martin, Implications of SUSY Model Building, Report of the DPF Working Group on "Electroweak Symmetry Breaking and Beyond the Standard Model",March 1995, hep-ph/9504324.

[7] See http://www-spires.slac.stanford.edu/find/hep.

[8] M. Drees, An Introduction to Supersymmetry, Lectures given at Inauguration Conference of the Asia Pacific Center for Theoretical Physics (APCTP), Seoul, Korea, 4-19 Jun 1996, hep-ph/9611409.

[9] H. Baer, et. al., Low Energy Supersymmetry Phenomenology, Report of the DPF Working Group on "Electroweak Symmetry Breaking and Beyond the Standard Model", March 1995, hep-ph/9503479.

[10] J. Lopez, Rept. Prog. Phys. 59:819-865,1996.

[11] A.H. Chamseddine, R. Arnowitt, and P. Nath, Phys. Rev. Lett. 49, 970 (1982); R. Barbieri, S. Ferrora, and C.A. Savoy, Phys. Lett. B119, 343 (1982); L. Hall, J. Lykken, and S. Weinberg, Phys. Rev. D27, 2359 (1983). For a recent review, see R. Arnowitt and P. Nath, "Supersymmetry and Supergravity: Phenomenology and Grand Unification," Proceedings of VII $^{\text {th }}$ J.A. Swieca Summer School, Campos de Jordao, Brazil, 1993 (World Scientific, Singapore, 1994). 
[12] R. Phillips and V. Barger, Collider Physics, Addison-Wesley (1987).

[13] L.E. Ibañez, C. Lopez, and C. Muoñz, Nucl. Phys. B256, 218 (1985); G.G. Ross and R.G. Roberts, Nucl. Phys. B377, 571 (1992); R. Arnowitt and P. Nath, Phys. Rev. Lett. 69, 725 (1992); S. Kelley, J.L. Lopez, D.V. Nanopoulos, H. Pois, and K. Yuan, Nucl. Phys. B398, 31 (1993); G.L. Kane, C. Kolda, L. Roszkowski, and J.D. Wells, Phys. Rev. D49, 6173 (1994). In this analysis we use formulae in H.Baer et al., Phys. Rev. D47, 2739 (1993).

[14] M.Drees and M.Nojiri, Nucl. Phys. B369, 54 (1992).

[15] See the references in G.L. Kane, HEPPH-9709318. Invited talk at 5th International Conference on Supersymmetries in Physics (SUSY 97), Philadelphia, PA, 27-31 May 1997.

[16] For example T.S. Kosmas and J.D. Vergados, Phys. Rev. D55:1752-1764 (1997).

[17] G. Anderson, private communication.

[18] J. Kubo, M. Mondragon and G. Zoupanos, Nucl.Phys. B424:291-307 (1994).

[19] D. Griffiths, Introduction to Elementary Particles. John Wiley \& Sons, Inc, 1987.

[20] F. Halzen and A. Martin, Quarks \& Leptons: An Introductory Course in Modern Particle Physics, John Wiley \& Sons, Inc., 1984.

[21] F. Abe, et al. (The CDF Collaboration), Phys. Rev. Lett. 76:2006-2010, (1996).

[22] F. Abe, et al. (The CDF Collaboration), Phys. Rev. Lett. 75 1017, 1995. F. Abe, et al. (The CDF Collaboration), in preparation.

[23] F. Abe, et al., Phys. Rev. Lett. 76, 4307 (1996).

[24] G. Anderson and D. Castaño, Phys. Letters B347, 300 (1995).

[25] G. Anderson and D. Castaño, Phys. Rev. D52, 1693 (1995).

[26] J. Huston, FERMILAB-TM-1909 (1994).

[27] For a description of the original CDF detector, see F. Abe, et al., (The CDF Collaboration), Nucl. Instrum. Methods A271, 387 (1988) and the references within.

[28] For a description of the CDF upgrades, see F. Abe, et al., (The CDF Collaboration), Phys. Rev. D50, 2966 (1994); D. Amidei, et al., Nucl. Instrum. Methods A350, 73 (1994) and the references within.

[29] A. Beretvas, et. al, CDF Note 3599 (1995). 
[30] F. Abe, et al., (The CDF Collaboration), Phys.Rev. D50:2966, 1994.

[31] S. Kuhlmann, et al., CDF Note 413 (1986); CDF Note 282.

[32] J.P. Done et al., CDF Note 4218 (1997).

[33] F. Abe, et. al, (The CDF Collaboration), Phys. Rev. D 522624 (1994).

[34] S. Kopp and C. Grosso-Pilcher, CDF Note 1992 (1993).

[35] M. Krasberg and T. LeCompte, CDF Note 2032 (1993).

[36] H. Baer, F.E. Paige, S.D. Protopopescu, and X. Tata, "Simulating Supersymmetry with ISAJET 7.0/ISASUSY 1.0," Proceedings of Workshop on Physics at Current Accelerators and the Supercollider, eds. J. Hewett, A. White and D. Zeppenfeld (Argonne National Laboratory, 1993).

[37] S. Tether, CDF Note 3566 (1996).

[38] The trigger efficiencies come from a variety of CDF Notes: CDF-2391, CDF-2391, CDF-2367, CDF-2186, CDF-3622, CDF-2435, XCES efficiency from CDF-2795, CDF-3060, CDF-2367, CDF-4017, CDF-2954, CDF-4076, CDF-3622, CDF-2596, CDF-2596, CDF-2367, CDF-2367, CDF-3532, CDF-3532, CDF-2191 and CDF-3028.

[39] B. Tannenbaum, CDF Note 2962 (1995).

[40] Andy Beretvas, private communication.

[41] J.P. Done et al., CDF Note 4182 (1997).

[42] K. Byrum and A.B. Wicklund, CDF Note 3665 (1996). The Level 2 trigger efficiency for CEM_9_SEED_9_SH_7_CFT_9_2_V2 in Run 1A is $\epsilon_{L 2}=\left(92.4 \pm 1.7_{-4.7}^{+3.0}\right) \%$. We shift the Run $1 \mathrm{~A}$ trigger efficiency curve by $1 \mathrm{GeV}$ and fold with XCES efficiencies (100\% at plateau) from CDF Note 2795; As for the Level 1 trigger efficiency, we take the Run $1 \mathrm{~A}$ result $\left(\epsilon_{L 1}=\right.$ $(99.2 \pm 0.1) \%)$ from CDF Note 2391 and shift the curve by $1 \mathrm{GeV}$ for Run IB; As for the Level 3 trigger efficiency for Run IB, we take the Run 1A result $\left(\epsilon_{L 3}=(95.2 \pm 1.5) \%\right)$ from CDF Note 2596; The total efficiency of $\mathrm{L} 1 * \mathrm{~L} 2 * \mathrm{~L} 3$ is $\left(87.3_{-4.9}^{+3.5}\right.$ (stat.+syst.))\%.

[43] J.P. Done et al., CDF 4017 (1997). The Level 2 trigger efficiency in Run IB is $\epsilon_{L 2}=(95.1 \pm 1.2 \pm$ $0.8) \%$; As for the Level 1 trigger efficiency, we have the Run IB result $\left(\epsilon_{L 1}=(93.1 \pm 1.1 \pm 1.0) \%\right)$ from CDF Note 3028; As for the Level 3 trigger efficiency, we take the Run 1A results $\left(\epsilon_{L 3}\right.$ $=(98.4 \pm 0.4 \pm 2.0) \%)$ from CDF Note 2596 for Run IB; The total efficiency of $\mathrm{L} 1 * \mathrm{~L} 2 * \mathrm{~L} 3$ is $(87.1 \pm 2.9$ (stat.+syst.))\% . 
[44] B. Tannenbaum and T. Kamon, CDF Note 3538 (1996).

[45] Y. Kato, D.Sc. Dissertation, (1996).

[46] B. Abbott, et al., hep-ex/9705015 (1997).

[47] Glen Cowen, CERN Particle Physics Seminar, 25 February 1997, http://alephwww.cern.ch/ALPUB/seminar/cowen-172-jam/cowan.html.

[48] J. Lopez, D. Nanopoulos, A. Zichichi, Phys. Rev. D52, 4178 (1995) and D53 5253 (1996).

[49] H. Baer, et al Phys. Rev. D51,1046 (1995) and references within.

[50] R. Blair, et al (The CDF II Collaboration), The CDF II Detector Technical Design Report, FERMILAB-Pub-96/390-E (1996).

[51] R. Barbieri and G. F. Giudice, Nucl. Phys, B306, 63 (1988); J. Ellis, K. Enqvist, D.V. Nanopoulos, and F. Zwirner, Mod. Phys. Lett. A1, 57 (1986).

[52] F. Abe, et. al, (The CDF Collaboration), Phys. Rev. D 524784 (1995). 